\title{
11/15/17 Complexes as Molecular Models for Metal Halide Double Perovskite Materials
}

\author{
Jakob Möbs, Johanna Heine* \\ Department of Chemistry and Material Sciences Center, Philipps-Universität Marburg, Hans- \\ Meerwein-Straße, 35043 Marburg, Germany.
}

\section{Additional Experimental Procedures}

$\mathrm{SbI}_{3}$ and $\mathrm{BiI}_{3}$ were synthesized from the elements according to literature procedures. ${ }^{1,2} \mathrm{AgCl}$ and $\mathrm{AgBr}$ were prepared by mixing aqueous solutions of $\mathrm{AgNO}_{3}$ and $\mathrm{KCl}$ or $\mathrm{KBr}$. The resulting precipitate was isolated by centrifugation, washed with water, dried under vacuum and stored in the dark. Copper halides, as well as $\mathrm{AgI}, \mathrm{BiBr}_{3}, \mathrm{BiCl}_{3}$ and $\mathrm{PPh}_{3}$ were used as supplied from commercial sources. We note that pentel halides $\mathrm{EX}_{3}$ need be stored under dry conditions to avoid hydrolysis towards oxyhalides and commercial products should be tested for the presence of these decomposition products before use. Copper halides should be stored under inert conditions to avoid oxidation towards $\mathrm{Cu}(\mathrm{II})$ salts. Silver halides should be stored in the dark due to their light sensitivity. Reactions were performed under inert conditions using acetone $\left(\mathrm{Me}_{2} \mathrm{CO}\right)$, acetonitrile $(\mathrm{MeCN})$ or 1,2-dimethoxyethane (DME) dried over $3 \AA$ mole sieve to avoid the formation of hydrolysis or oxidation products during the reaction in solution.

$\left[\mathbf{B i A g}_{2} \mathbf{B r}_{\mathbf{5}}\left(\mathbf{P P h}_{3}\right)_{4}\left(\mathbf{M e C N}_{\mathbf{0 . 7}^{-7}}\right.\right.$ (3a): $67 \mathrm{mg} \mathrm{BiBr}{ }_{3}(0.15 \mathrm{mmol}, 1 \mathrm{eq}), 56 \mathrm{mg} \mathrm{AgBr}(0.3 \mathrm{mmol}, 2 \mathrm{eq})$ and $157 \mathrm{mg}$ of $\mathrm{PPh}_{3}(0.6 \mathrm{mmol}, 4 \mathrm{eq})$ were suspended in $10 \mathrm{~mL}$ of acetonitrile and heated to $90{ }^{\circ} \mathrm{C}$ for $90 \mathrm{~min}$. A voluminous yellow precipitate of $\mathbf{3 a}$ was obtained, filtered off, washed three times with $4 \mathrm{~mL}$ of cold acetonitrile and dried in vacuum. Yield: $178 \mathrm{mg}$ (62\%). CHN (calculated for $\left.\left[\mathrm{BiAg}_{2} \mathrm{Br}_{5}\left(\mathrm{PPh}_{3}\right)_{4}(\mathrm{MeCN})_{0.7}\right]\right): \mathrm{C} 45.90$ (46.35), H 3.202 (3.29), N 0.57 (0.52). Yellow single crystals of 3a were grown from the filtrate within 2 days at room temperature. 
Powder patterns of the dried precipitate accord well with the simulation from SCXRD data and thermal analysis confirms the partial occupancy of the MeCN ligand observed in the SCXRD data and CHN analysis (see below for details).

$\left[\mathbf{B i A g}_{2} \mathbf{B r}_{5}\left(\mathbf{P P h}_{3}\right)_{4}\left(\mathbf{M e}_{2} \mathbf{C O}\right)\right]$ (3b): $67 \mathrm{mg} \mathrm{BiBr} 3(0.15 \mathrm{mmol}, 1$ eq), $56 \mathrm{mg} \mathrm{AgBr}(0.3 \mathrm{mmol}, 2$ eq) and $157 \mathrm{mg}$ of $\mathrm{PPh}_{3}(0.6 \mathrm{mmol}, 4 \mathrm{eq})$ were suspended in $10 \mathrm{~mL}$ of acetone and heated to $70{ }^{\circ} \mathrm{C}$ for $90 \mathrm{~min}$, resulting in a yellow solution and a voluminous yellow precipitate of $\mathbf{3 b}$. The reaction mixture was filtered while hot and the precipitate was washed three times with $4 \mathrm{~mL}$ of cold acetone and three times with $10 \mathrm{~mL}$ of hexane and dried in vacuum. Yield: $181 \mathrm{mg}$ (62\%). CHN (calculated for $\left.\left[\mathrm{BiAg}_{2} \mathrm{Br}_{5}\left(\mathrm{PPh}_{3}\right)_{4}\left(\mathrm{Me}_{2} \mathrm{CO}\right)_{0.76}\right]\right): \mathrm{C} 46.54$ (46.53), H 3.392 (3.39), $\mathrm{N} 0$ (0).

Powder patterns of the dried precipitate accord well with the simulation from SCXRD data of $\mathbf{3 a}$ indicating that $\mathbf{3 a}$ and $\mathbf{3 b}$ are isostructural. Thermal analysis confirms the partial solvent loss observed in the CHN analysis of $\mathbf{3 b}$ (see below for details).

$\left[\mathbf{B i C u}_{3} \mathbf{B r}_{6}\left(\mathbf{P P h}_{3}\right)_{6}\right]$ (4): $67 \mathrm{mg} \mathrm{BiBr} 3(0.15 \mathrm{mmol}, 1.5 \mathrm{eq}), 43 \mathrm{mg} \mathrm{CuBr}(0.3 \mathrm{mmol}, 3 \mathrm{eq})$ were suspended in $10 \mathrm{~mL}$ of acetone. $157 \mathrm{mg}$ of $\mathrm{PPh}_{3}(0.6 \mathrm{mmol}, 6 \mathrm{eq})$ were added, resulting in a clear yellow solution. The reaction mixture was heated to $70{ }^{\circ} \mathrm{C}$ for $2 \mathrm{~h}$ and left to cool to room temperature. An orange precipitate of $\mathbf{4}$ had formed and was filtered off, washed two times with $5 \mathrm{~mL}$ methanol and dried in vacuum. Yield: $148 \mathrm{mg}$ ( $58 \%$ with respect to the limiting reagents $\mathrm{CuBr}$ and $\mathrm{PPh}_{3}$ ). $\mathrm{CHN}$ (calculated): C 52.88 (52.89), H 3.697 (3.70), N 0 (0).

Single crystals of 4 could be grown from the filtrate of the reaction mixture within $12 \mathrm{~h}$ at room temperature.

$\left[\mathbf{B i A g}_{3} \mathbf{B r}_{6}\left(\mathbf{P P h}_{3}\right)_{6}\right]$ (5a): $45 \mathrm{mg} \mathrm{BiBr}{ }_{3}(0.1 \mathrm{mmol}, 1 \mathrm{eq}), 56 \mathrm{mg} \mathrm{AgBr}(0.3 \mathrm{mmol}, 3 \mathrm{eq})$ and $157 \mathrm{mg}$ of $\mathrm{PPh}_{3}\left(0.6 \mathrm{mmol}, 6\right.$ eq) were suspended in $6 \mathrm{~mL}$ of DME and heated to $90{ }^{\circ} \mathrm{C}$ for $90 \mathrm{~min}$, resulting in a light yellow solution and a yellow precipitate of 5a. The reaction mixture was filtered while hot and the precipitate was washed with three $10 \mathrm{~mL}$ portions of pentane and dried in vacuum. Yield: $209 \mathrm{mg}$ (81 \%). CHN (calculated): C 49.83 (50.17), H 3.549 (3.51), N 0 (0).

Powder patterns of 5a accord well with the simulation of SCXRD data from Fenske's work. ${ }^{3}$ See below for details and thermal analysis data. 
$\left[\mathbf{B i A g}_{3} \mathbf{B r}_{6}\left(\mathbf{P P h}_{3}\right)_{6}\right] \cdot 4 \mathbf{M e C N}(\mathbf{5 b}): 45 \mathrm{mg} \mathrm{BiBr}{ }_{3}(0.1 \mathrm{mmol}, 1 \mathrm{eq}), 56 \mathrm{mg} \mathrm{AgBr}(0.3 \mathrm{mmol}, 3 \mathrm{eq})$ and $157 \mathrm{mg}$ of $\mathrm{PPh}_{3}(0.6 \mathrm{mmol}, 6 \mathrm{eq})$ were suspended in $20 \mathrm{~mL}$ of acetonitrile and heated to $85^{\circ} \mathrm{C}$ for $90 \mathrm{~min}$, resulting in a yellow solution and an inhomogeneous, light-colored precipitate. The reaction mixture was filtered while hot. The precipitate was shown to contain $\mathrm{AgBr}, \mathbf{3 a}$ and $\mathbf{5 a}$ via PXRD. Yellow single crystals of $\mathbf{5 b}$ grew in the filtrate along with unidentified colorless crystals, precluding the isolation of a pure bulk product.

$\left[\mathbf{B i C u}_{3} \mathbf{B r}_{3} \mathbf{I}_{3}\left(\mathbf{P P h}_{3}\right)_{6}\right](\mathbf{6}): 45 \mathrm{mg} \mathrm{BiBr}{ }_{3}(0.1 \mathrm{mmol}, 1 \mathrm{eq}), 57 \mathrm{mg} \mathrm{CuI}(0.3 \mathrm{mmol}, 3 \mathrm{eq})$ and $157 \mathrm{mg}$ of $\mathrm{PPh}_{3}(0.6 \mathrm{mmol}, 6 \mathrm{eq})$ were suspended in $10 \mathrm{~mL}$ of DME and heated to $85^{\circ} \mathrm{C}$ for $90 \mathrm{~min}$, resulting in a clear red solution that was filtered while hot. Within 5 days at $5{ }^{\circ} \mathrm{C}$ dark red single crystals of 6 formed in the filtrate. The mother liquor was decanted from the crystalline product, which was washed with three $4 \mathrm{~mL}$ portions of hexane and dried in vacuum. Yield: $126 \mathrm{mg}$ (49\%). CHN (calculated): C 50.06 (50.01), H 3.454 (3.50), N 0 (0). Semi-quantitative analysis using $\mu$-XRF confirms the presence of $\mathrm{Br}$ and $\mathrm{I}$ in a 1:1 ratio in the crystals of 6.

[SbCu $\left.\mathbf{S}_{3} \mathbf{I}_{6}\left(\mathbf{P P h}_{3}\right)_{6}\right]$ (7): $\left.100 \mathrm{mg} \mathrm{SbI}{ }_{3}(0.2 \mathrm{mmol}, 2 \mathrm{eq}), 57 \mathrm{mg} \mathrm{CuI} \mathrm{(0.3} \mathrm{mmol,} 3 \mathrm{eq}\right)$ and $157 \mathrm{mg}$ of $\mathrm{PPh}_{3}$ (0.6 mmol, 6 eq) were suspended in $10 \mathrm{~mL}$ of DME and heated to $85^{\circ} \mathrm{C}$ for $2 \mathrm{~h}$, resulting in a clear orange solution that was filtered while hot. Within 3 days at room temperature bright red single crystals of 7 formed in the filtrate. The mother liquor was decanted from the crystalline product, which was washed with three $2 \mathrm{~mL}$ portions of cold acetone and dried in vacuum. Yield: $23 \mathrm{mg}$ (9\% with respect to the limiting reagents $\mathrm{CuI}$ and $\mathrm{PPh}_{3}$ ). $\mathrm{CHN}$ (calculated): $\mathrm{C} 49.05$ (49.00), $\mathrm{H} 3.263$ (3.43), $\mathrm{N} 0$ (0).

$\left[\mathbf{B i A g}_{3} \mathbf{I}_{6}\left(\mathbf{P P h}_{3}\right)_{6}\right]$ (9a): The compound can be obtained by heating the acetonitrile solvate $\mathbf{9 b}$ (vide infra) for $16 \mathrm{~h}$ at $100^{\circ} \mathrm{C}$ in a vacuum. Powder patterns suggest a complete conversion (see SI for details), but CHN analysis (CHN (calculated): C 44.41 (45.23), H 3.094 (3.16), N 0.14 (0)) points to some decomposition upon extended heating.

Reactions in DME and acetone were investigated to obtain single crystalline or at least phase pure 9a, but solely yielded binary silver iodide-phosphine complexes.

$\left[\mathbf{B i A g}_{3} \mathbf{I}_{6}\left(\mathbf{P P h}_{3}\right)_{6}\right] \cdot \mathbf{4} \mathbf{M e C N}_{\mathbf{9 b}}$ : $118 \mathrm{mg} \mathrm{BiI}{ }_{3}(0.2 \mathrm{mmol}, 1 \mathrm{eq}), 140 \mathrm{mg} \mathrm{AgI}(0.6 \mathrm{mmol}, 3 \mathrm{eq})$ and $314 \mathrm{mg}$ of $\mathrm{PPh}_{3}(1.2 \mathrm{mmol}, 6 \mathrm{eq})$ were suspended in $40 \mathrm{~mL}$ of acetonitrile and heated to $90{ }^{\circ} \mathrm{C}$ for $2 \mathrm{~h}$, 
resulting in a deep orange solution and inhomogeneous grey and red precipitates that were identified as a mixture of metallic bismuth, $\left[\mathrm{Ag}_{4} \mathrm{I}_{4}\left(\mathrm{PPh}_{3}\right)_{4}\right]^{4}$ and $9 \mathbf{b}$ via PXRD. The reaction mixture was filtered while hot and the filtrate stored at $-26^{\circ} \mathrm{C}$ for 10 days. Red crystals of $9 \mathrm{~b}$ were isolated by decanting the mother liquor, washing with $3 \mathrm{~mL}$ of cold methanol and drying in vacuum. Yield: $89 \mathrm{mg}(15 \%)$. CHN (calculated): C 45.89 (45.95), H 3.425 (3.39), N 1,85 (1.85) 


\section{Additional Crystallographic Details}

Table S1: Crystallographic data for 1.

\begin{tabular}{ll}
\hline & 1 \\
\hline Empirical formula & $\mathrm{C}_{84} \mathrm{H}_{84} \mathrm{Bi}_{2} \mathrm{Cl}_{8} \mathrm{Cu}_{2} \mathrm{O}_{4} \mathrm{P}_{4}$ \\
Formula weight $/ \mathrm{g} \cdot \mathrm{mol}^{-1}$ & 2110.03 \\
Crystal color and shape & yellow block \\
Crystal size & $0.278 \times 0.249 \times 0.146$ \\
Crystal system & triclinic \\
Space group & $P \overline{1}$ \\
$a / \AA$ & $10.0540(6)$ \\
$b / \AA$ & $12.8932(7)$ \\
$c / \AA$ & $17.8018(9)$ \\
$\alpha /{ }^{\circ}$ & $70.159(2)$ \\
$\beta /{ }^{\circ}$ & $85.990(2)$ \\
$\gamma /{ }^{\circ}$ & $75.745(2)$ \\
$V / \AA^{3}$ & $2103.6(2)$ \\
$Z$ & 1 \\
$\rho_{\text {calc }} / \mathrm{g} \cdot \mathrm{cm}^{-3}$ & 1.666 \\
$\mu($ Mo & \\
measurement temp. $/ \mathrm{K}$ & 5.047 \\
$\mathrm{Absorption}$ correction type & 100 \\
Min/max transmission & multi-scan \\
$2 \Theta$ range ${ }^{\circ}$ & $0.334 / 0.526$ \\
No. of measured reflections & $4.752-50.674$ \\
No. of independent reflections & 45370 \\
$R($ int $)$ & 7686 \\
No. of indep. reflections $(I>2 \sigma(I))$ & 7158 \\
No. of parameters & 473 \\
$R_{1}(I>2 \sigma(I))$ & 0.0176 \\
$w R_{2}($ all data) & 0.0415 \\
$S$ (all data) & 1.129 \\
$\Delta \rho_{\text {max }}, \Delta \rho_{\text {min }} / \mathrm{e} \cdot \AA-3$ & $0.51 /-0.84$ \\
\hline & \\
\hline & \\
\hline
\end{tabular}

Details of crystal structure measurement and refinement: Single crystal X-ray determination was performed at $100 \mathrm{~K}$ on a Bruker Quest D8 diffractometer with microfocus MoKa radiation and a Photon 100 (CMOS) detector. 

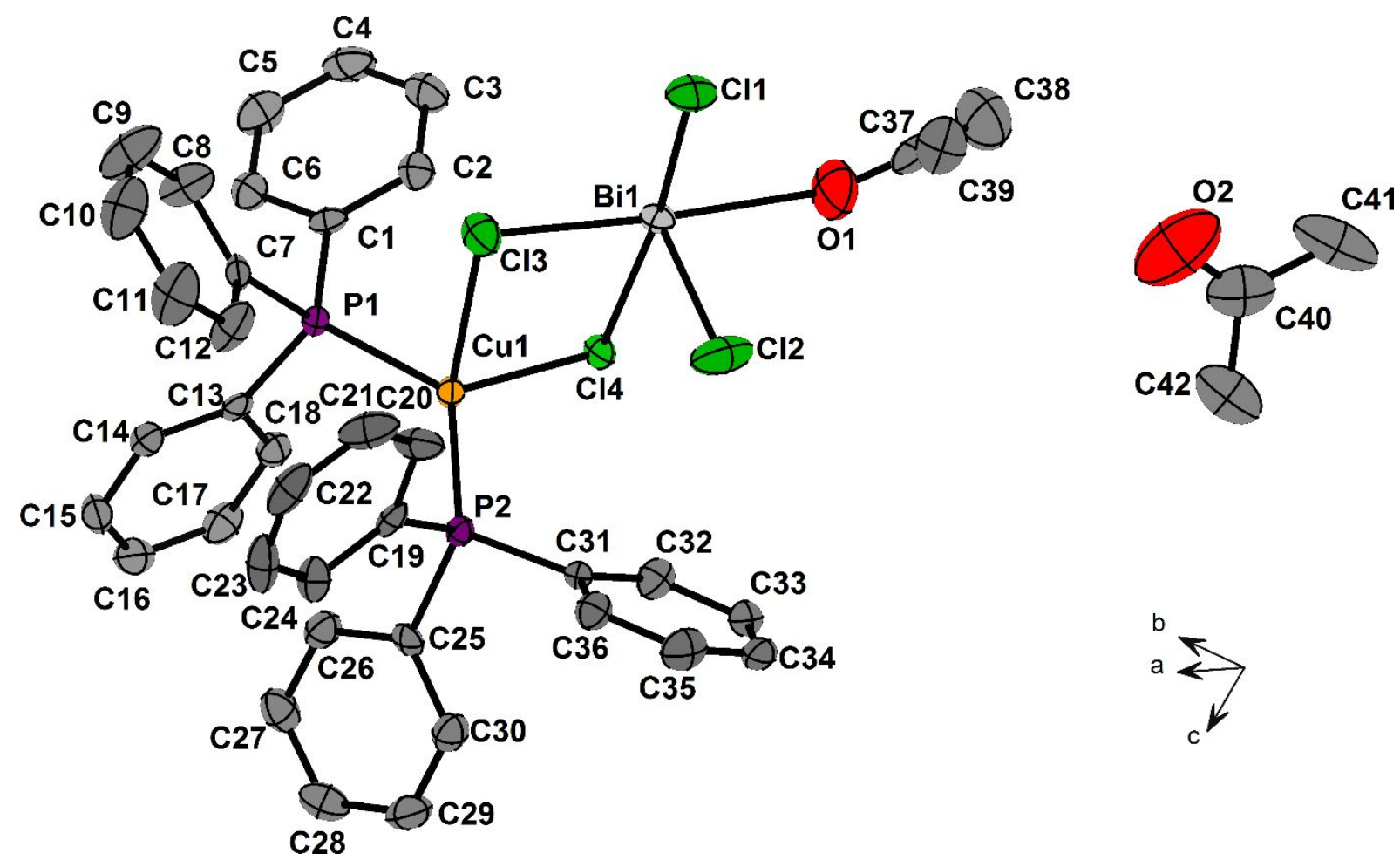

Figure S1: Asymmetric unit of 1, ellipsoids at $80 \%$ probability. 
Table S2: Crystallographic data for 2.

\begin{tabular}{|c|c|}
\hline & 2 \\
\hline Empirical formula & $\mathrm{C}_{75} \mathrm{H}_{66} \mathrm{Ag}_{2} \mathrm{BiCl}_{5} \mathrm{OP}_{4}$ \\
\hline Formula weight $/ \mathrm{g} \cdot \mathrm{mol}^{-1}$ & 1709.12 \\
\hline Crystal color and shape & colorless plate \\
\hline Crystal size & $0.195 \times 0.068 \times 0.055$ \\
\hline Crystal system & triclinic \\
\hline Space group & $P \overline{1}$ \\
\hline$a / \AA$ & $10.1773(4)$ \\
\hline$b / \AA$ & $12.9770(5)$ \\
\hline$c / \AA$ & $14.3760(6)$ \\
\hline$\alpha / 0$ & $68.6370(10)$ \\
\hline$\beta /{ }^{\circ}$ & $80.0670(10)$ \\
\hline$\gamma /{ }^{\circ}$ & $73.4730(10)$ \\
\hline$V / \AA^{3}$ & $1690.09(12)$ \\
\hline$Z$ & 1 \\
\hline$\rho_{\text {calc }} / \mathrm{g} \cdot \mathrm{cm}^{-3}$ & 1.679 \\
\hline$\mu\left(\mathrm{Mo}_{\mathrm{K} \alpha}\right) / \mathrm{mm}^{-1}$ & 3.508 \\
\hline measurement temp. $/ \mathrm{K}$ & 100 \\
\hline Absorption correction type & multi-scan \\
\hline Min/max transmission & $0.6647 / 0.7452$ \\
\hline $2 \Theta$ range $/{ }^{\circ}$ & $4.75-50.686$ \\
\hline No. of measured reflections & 40452 \\
\hline No. of independent reflections & 6164 \\
\hline$R($ int $)$ & 0.0496 \\
\hline No. of indep. reflections $(I>2 \sigma(I))$ & 5399 \\
\hline No. of parameters & 419 \\
\hline$R_{1}(I>2 \sigma(I))$ & 0.0265 \\
\hline$w R_{2}$ (all data) & 0.0542 \\
\hline$S$ (all data) & 1.035 \\
\hline$\Delta \rho_{\max }, \Delta \rho_{\min } / \mathrm{e} \cdot \AA^{-3}$ & $0.74 /-0.75$ \\
\hline
\end{tabular}

Details of crystal structure measurement and refinement: Single crystal X-ray determination was performed at $100 \mathrm{~K}$ on a Bruker Quest D8 diffractometer with microfocus MoKa radiation and a Photon 100 (CMOS) detector. The bismuth atom and one of the bridging chlorine atoms are disordered about the inversion center and thus have an occupancy of 0.5 . It is not possible to refine the structure with the $\mathrm{Bi}$ atom sitting at $\left(\begin{array}{lll}1 & 1 / 2 & 0\end{array}\right)$ as this results in very large residual electron densities. Overall, the $\mathrm{BiCl}_{5}$ (acetone) unit is found in two different orientations with regard to the terminal $\mathrm{Cl}$ and acetone ligands (both with occupancies of 0.5 ). It is not possible to use the PART command to model these two positions, but it is possible to clearly assign connectivities by choosing reasonable bond lengths and angles. Four carbon atoms on one of the phenyl rings were refined disordered about two positions with 0.5 occupancy each. Disordered carbon atoms were refined isotropically. 


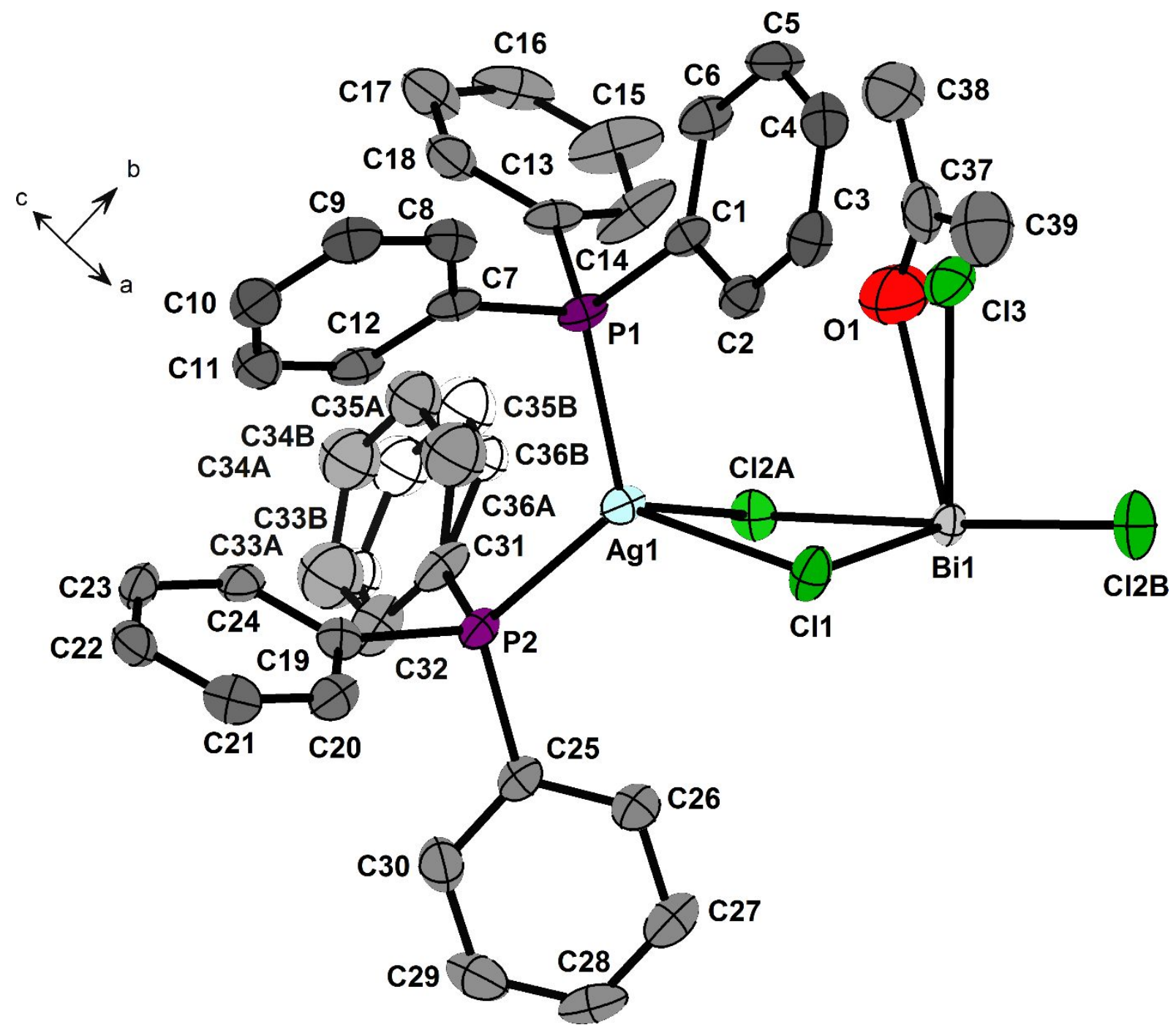

Figure S2: Asymmetric unit of 2, ellipsoids at $80 \%$ probability. 
Table S3: Crystallographic data for 3a.

\begin{tabular}{|c|c|}
\hline & $\mathbf{3 a}$ \\
\hline Empirical formula & $\mathrm{C}_{73.33} \mathrm{H}_{62} \mathrm{Ag}_{2} \mathrm{BiBr}_{5} \mathrm{~N}_{0.67} \mathrm{P}_{4}$ \\
\hline Formula weight $/ \mathrm{g} \cdot \mathrm{mol}^{-1}$ & 1900.72 \\
\hline Crystal color and shape & yellow block \\
\hline Crystal size & $0.355 \times 0.170 \times 0.161$ \\
\hline Crystal system & triclinic \\
\hline Space group & $P \overline{1}$ \\
\hline$a / \AA$ & $10.3017(4)$ \\
\hline$b / \AA$ & $12.8441(5)$ \\
\hline$c / \AA$ & $14.4848(5)$ \\
\hline$\alpha /{ }^{\circ}$ & $69.6050(10)$ \\
\hline$\beta /{ }^{\circ}$ & $80.4460(10)$ \\
\hline$\gamma /{ }^{\circ}$ & $73.585(2)$ \\
\hline$V / \AA^{3}$ & $1718.34(11)$ \\
\hline$Z$ & 1 \\
\hline$\rho_{\text {calc }} / \mathrm{g} \cdot \mathrm{cm}^{-3}$ & 1.837 \\
\hline$\mu\left(\mathrm{Mo}_{\mathrm{K} \alpha}\right) / \mathrm{mm}^{-1}$ & 6.160 \\
\hline measurement temp. /K & 100 \\
\hline Absorption correction type & multi-scan \\
\hline Min/max transmission & $0.4383 / 0.7452$ \\
\hline $2 \Theta$ range $/{ }^{\circ}$ & $4.716-50.622$ \\
\hline No. of measured reflections & 13496 \\
\hline No. of independent reflections & 6219 \\
\hline$R$ (int) & 0.0179 \\
\hline No. of indep. reflections $(I>2 \sigma(I))$ & 5803 \\
\hline No. of parameters & 394 \\
\hline$R_{1}(I>2 \sigma(I))$ & 0.0278 \\
\hline$w R_{2}$ (all data) & 0.0665 \\
\hline$S$ (all data) & 1.082 \\
\hline$\Delta \rho_{\max }, \Delta \rho_{\min } / \mathrm{e} \cdot \AA^{-3}$ & $1.00 /-3.67$ \\
\hline
\end{tabular}

Details of crystal structure measurement and refinement: Single crystal X-ray determination was performed at $100 \mathrm{~K}$ on a Bruker Quest D8 diffractometer with microfocus MoK $\alpha$ radiation and a Photon 100 (CMOS) detector. The terminal $\mathrm{Br}$ atom and acetonitrile ligand of the $\mathrm{BiBr}_{5}$ (acetonitrile) unit are disordered, resulting in occupancies of 0.5 and 0.333 , respectively. While a 0.5 occupancy would be expected on the acetonitrile ligand, the partial occupancy is confirmed by CHN and TGA measurements. Four carbon atoms on one of the phenyl rings were refined disordered about two positions with 0.5 occupancy each. Disordered atoms were refined isotropically. 


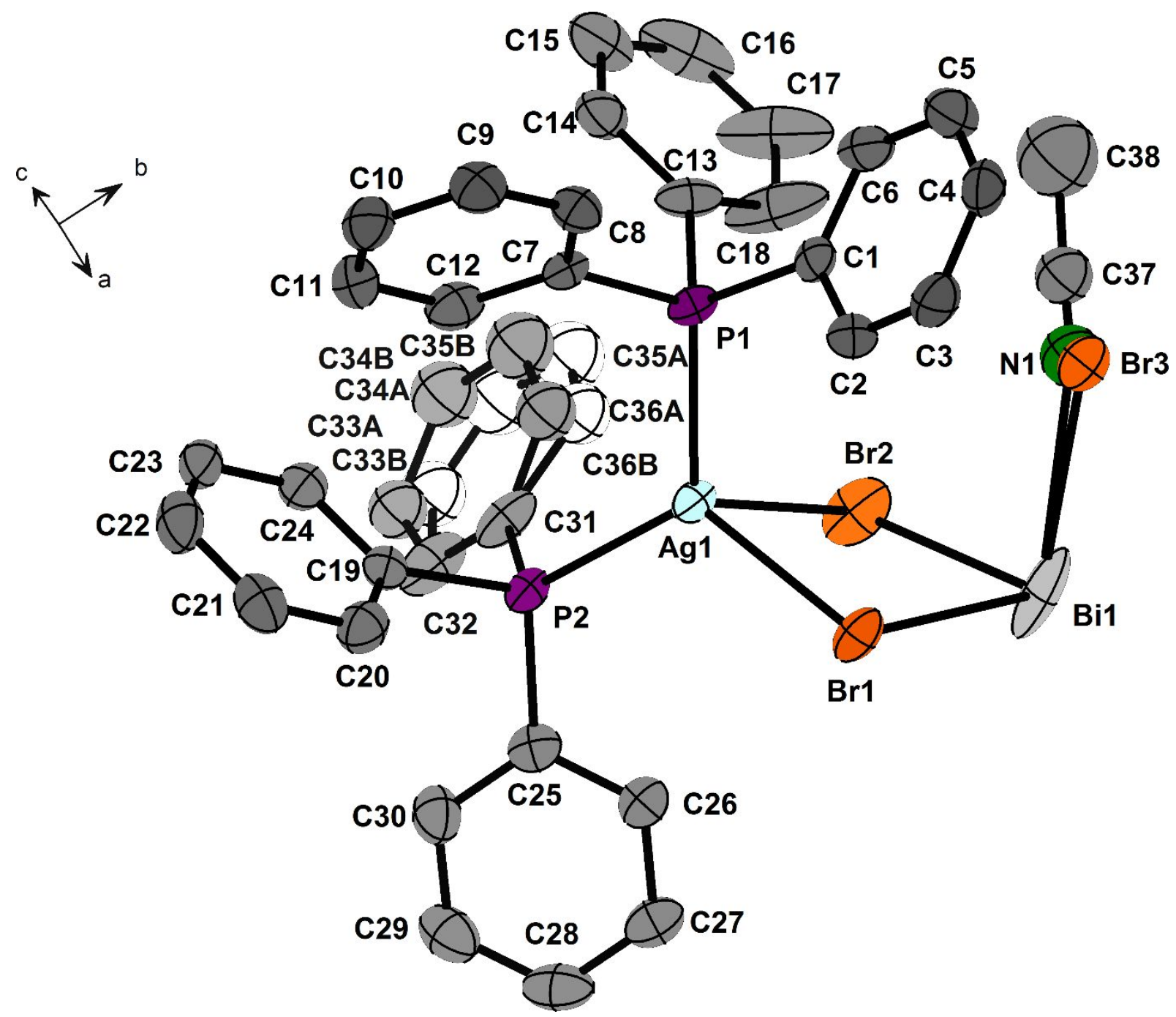

Figure S3: Asymmetric unit of 3a, ellipsoids at $80 \%$ probability. 
Table S4: Crystallographic data for 4.

\begin{tabular}{|c|c|}
\hline & 4 \\
\hline Empirical formula & $\mathrm{C}_{108} \mathrm{H}_{90} \mathrm{BiBr}_{6} \mathrm{Cu}_{3} \mathrm{P}_{6}$ \\
\hline Formula weight $/ \mathrm{g} \cdot \mathrm{mol}^{-1}$ & 2452.67 \\
\hline Crystal color and shape & orange plank \\
\hline Crystal size & $0.43 \times 0.22 \times 0.06$ \\
\hline Crystal system & orthorhombic \\
\hline Space group & $P n a 2_{1}$ \\
\hline$a / \AA$ & $30.9946(7)$ \\
\hline$b / \AA$ & $17.5621(4)$ \\
\hline$c / \AA$ & $17.5344(4)$ \\
\hline$\alpha /{ }^{\circ}$ & 90 \\
\hline$\beta /{ }^{\circ}$ & 90 \\
\hline$\gamma /{ }^{\circ}$ & 90 \\
\hline$V / \AA^{3}$ & $9544.5(4)$ \\
\hline$Z$ & 4 \\
\hline$\rho_{\text {calc }} / \mathrm{g} \cdot \mathrm{cm}^{-3}$ & 1.707 \\
\hline$\mu\left(\mathrm{Mo}_{\mathrm{K} \alpha}\right) / \mathrm{mm}^{-1}$ & 5.159 \\
\hline measurement temp. $/ \mathrm{K}$ & 100 \\
\hline Absorption correction type & multi-scan \\
\hline Min/max transmission & $0.0118 / 0.0335$ \\
\hline $2 \Theta$ range $/{ }^{\circ}$ & $2.628-53.518$ \\
\hline No. of measured reflections & 62425 \\
\hline No. of independent reflections & 18925 \\
\hline$R($ int $)$ & 0.0955 \\
\hline No. of indep. reflections $(I>2 \sigma(I))$ & 11591 \\
\hline No. of parameters & 1118 \\
\hline$R_{1}(I>2 \sigma(I))$ & 0.0415 \\
\hline$w R_{2}$ (all data) & 0.0766 \\
\hline$S$ (all data) & 0.713 \\
\hline$\Delta \rho_{\max }, \Delta \rho_{\min } / \mathrm{e}^{-} \AA^{-3}$ & $1.78 /-0.64$ \\
\hline
\end{tabular}

Details of crystal structure measurement and refinement: Single crystal X-ray determination was performed at $100 \mathrm{~K}$ on a STOE IPDS2 diffractometer equipped with an imaging plate detector system using MoKa radiation with graphite monochromatization. 


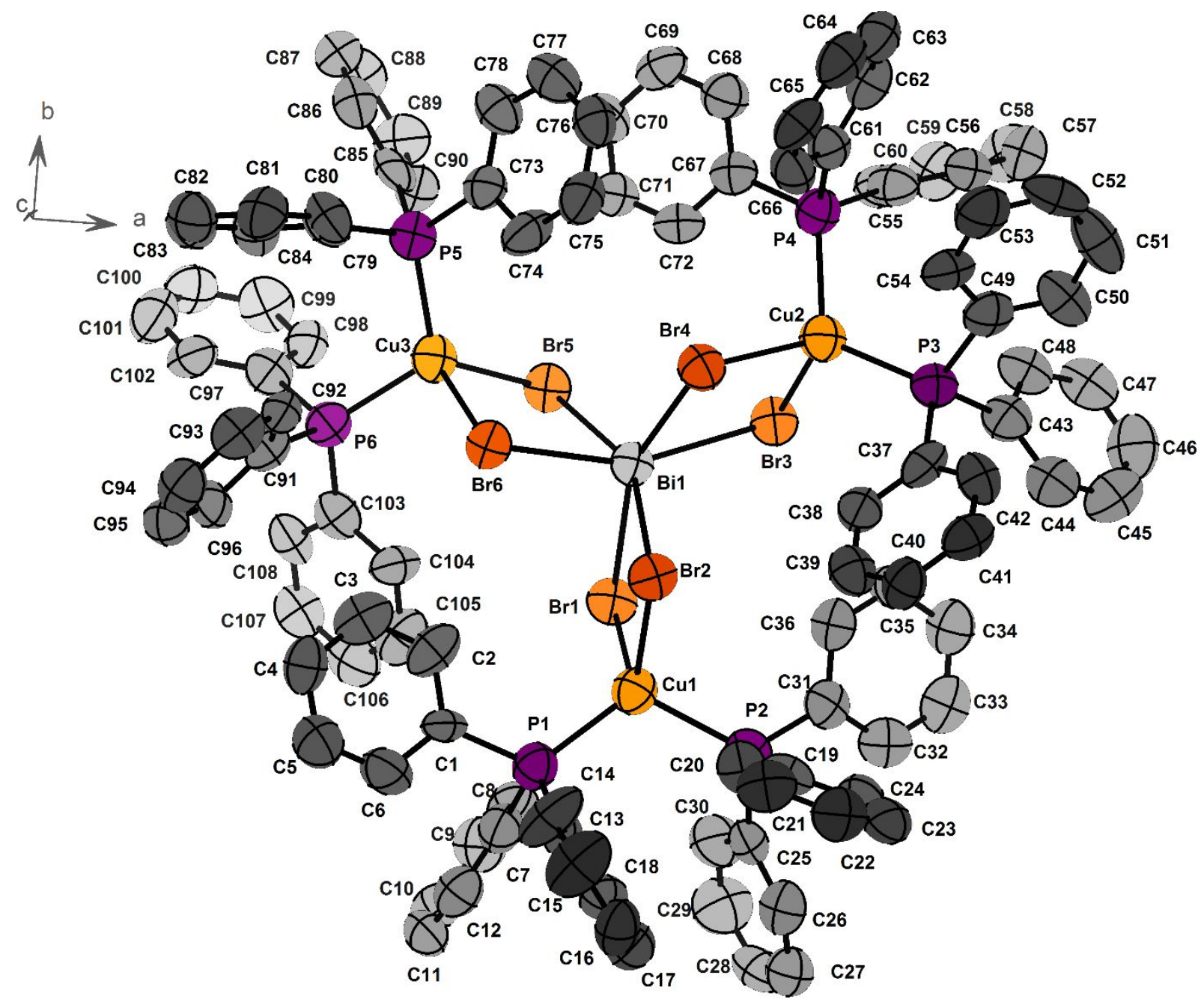

Figure S4: Asymmetric unit of 4, ellipsoids at $80 \%$ probability. 
Table S5: Crystallographic data for 5b.

\begin{tabular}{|c|c|}
\hline & $5 \mathbf{b}$ \\
\hline Empirical formula & $\mathrm{C}_{116} \mathrm{H}_{102} \mathrm{Ag}_{3} \mathrm{BiBr}_{6} \mathrm{~N}_{4} \mathrm{P}_{6}$ \\
\hline Formula weight $/ \mathrm{g} \cdot \mathrm{mol}^{-1}$ & 2749.91 \\
\hline Crystal color and shape & yellow block \\
\hline Crystal size & $0.200 \times 0.168 \times 0.164$ \\
\hline Crystal system & trigonal \\
\hline Space group & $R 3 c$ \\
\hline$a / \AA$ & $19.3720(9)$ \\
\hline$b / \AA$ & $19.3720(9)$ \\
\hline$c / \AA$ & $49.531(2)$ \\
\hline$\alpha / 0$ & 90 \\
\hline$\beta /{ }^{\circ}$ & 90 \\
\hline$\gamma /{ }^{\circ}$ & 120 \\
\hline$V / \AA^{3}$ & $16097.5(17)$ \\
\hline$Z$ & 6 \\
\hline$\rho_{\text {calc }} / \mathrm{g} \cdot \mathrm{cm}^{-3}$ & 1.702 \\
\hline$\mu\left(\mathrm{Mo}_{\mathrm{K} \alpha}\right) / \mathrm{mm}^{-1}$ & 4.548 \\
\hline measurement temp. /K & 100 \\
\hline Absorption correction type & multi-scan \\
\hline Min/max transmission & $0.6596 / 0.7452$ \\
\hline $2 \Theta$ range $/{ }^{\circ}$ & $4.876-50.666$ \\
\hline No. of measured reflections & 54814 \\
\hline No. of independent reflections & 6384 \\
\hline$R($ int $)$ & 0.0382 \\
\hline No. of indep. reflections $(I>2 \sigma(I))$ & 6111 \\
\hline No. of parameters & 410 \\
\hline$R_{1}(I>2 \sigma(I))$ & 0.0179 \\
\hline$w R_{2}$ (all data) & 0.0357 \\
\hline$S$ (all data) & 0.987 \\
\hline$\Delta \rho_{\max }, \Delta \rho_{\min } / \mathrm{e} \cdot \AA^{-3}$ & $0.34 /-0.48$ \\
\hline
\end{tabular}

Details of crystal structure measurement and refinement: Single crystal X-ray determination was performed at $100 \mathrm{~K}$ on a Bruker Quest D8 diffractometer with microfocus MoK $\alpha$ radiation and a Photon 100 (CMOS) detector. A total of four disordered molecules of acetonitrile per formula unit on two positions can be located. One molecule is disordered about the threefold axis in such a way that an occupancy of $1 / 3$ has to be chosen for the nitrogen and the terminal carbon atom. The second position of acetonitrile molecules features a partial disorder in the $\mathrm{C} \equiv \mathrm{N}$ group with occupancies of 0.5 and 0.5 . Disordered atoms were refined isotropically. 


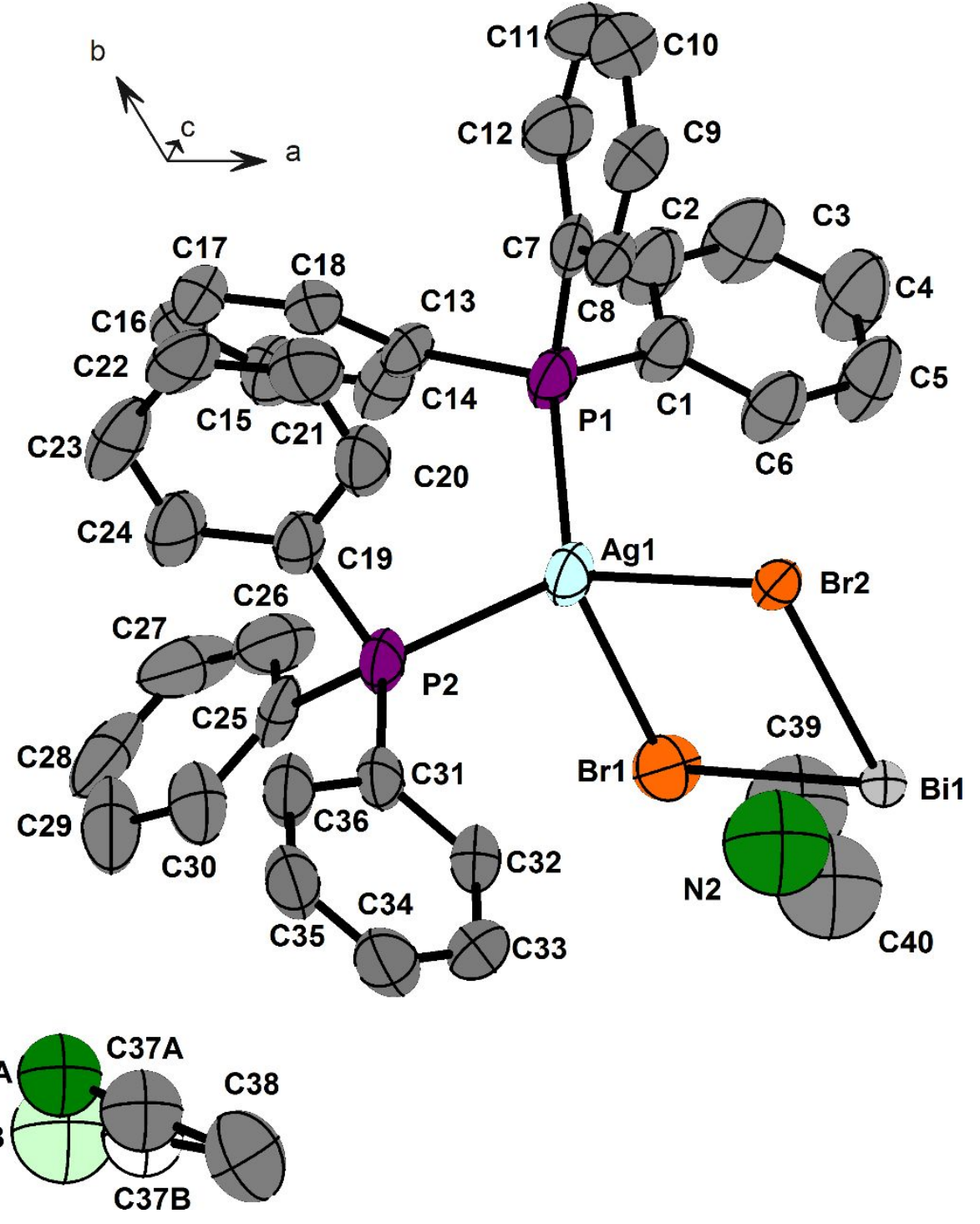

Figure S5: Asymmetric unit of 5b, ellipsoids at $80 \%$ probability. 
Table S6: Crystallographic data for 6.

\begin{tabular}{|c|c|}
\hline & 6 \\
\hline Empirical formula & $\mathrm{C}_{108} \mathrm{H}_{90} \mathrm{BiBr}_{3.11} \mathrm{Cu}_{3} \mathrm{I}_{2.89} \mathrm{P}_{6}$ \\
\hline Formula weight $/ \mathrm{g} \cdot \mathrm{mol}^{-1}$ & 2588.36 \\
\hline Crystal color and shape & red hexagon \\
\hline Crystal size & $0.269 \times 0.205 \times 0.190$ \\
\hline Crystal system & orthorhombic \\
\hline Space group & $\operatorname{Pna}_{1}$ \\
\hline$a / \AA$ & $31.0999(12)$ \\
\hline$b / \AA$ & $17.6930(7)$ \\
\hline$c / \AA$ & $17.6580(7)$ \\
\hline$\alpha /{ }^{\circ}$ & 90 \\
\hline$\beta /^{\circ}$ & 90 \\
\hline$\gamma /{ }^{\circ}$ & 90 \\
\hline$V / \AA^{3}$ & $9716.3(7)$ \\
\hline$Z$ & 4 \\
\hline$\rho_{\text {calc }} / \mathrm{g} \cdot \mathrm{cm}^{-3}$ & 1.769 \\
\hline$\mu\left(\mathrm{Mo}_{\mathrm{K} \alpha}\right) / \mathrm{mm}^{-1}$ & 4.798 \\
\hline measurement temp. $/ \mathrm{K}$ & 100 \\
\hline Absorption correction type & multi-scan \\
\hline Min/max transmission & $0.5811 / 0.7452$ \\
\hline $2 \Theta$ range $/{ }^{\circ}$ & $4.554-50.652$ \\
\hline No. of measured reflections & 97508 \\
\hline No. of independent reflections & 17488 \\
\hline$R$ (int) & 0.0580 \\
\hline No. of indep. reflections $(I>2 \sigma(I))$ & 15961 \\
\hline No. of parameters & 1123 \\
\hline$R_{1}(I>2 \sigma(I))$ & 0.0251 \\
\hline$w R_{2}$ (all data) & 0.0444 \\
\hline$S$ (all data) & 0.902 \\
\hline$\Delta \rho_{\max }, \Delta \rho_{\min } / \mathrm{e} \cdot \AA^{-3}$ & $0.45 /-0.43$ \\
\hline
\end{tabular}

Details of crystal structure measurement and refinement: Single crystal X-ray determination was performed at $100 \mathrm{~K}$ on a Bruker Quest D8 diffractometer with microfocus MoKa radiation and a Photon 100 (CMOS) detector. EADP and EXYZ commands were used to model the mixed occupancy of the halogen atom positions. The $\mathrm{Br} / \mathrm{I}$ ratio on each position was refined freely. The maximum observed iodine occupancy was 0.64 , the minimum occupancy 0.24 . Overall the resulting halogen composition matched well with CHN and X-Ray-fluorescence measurements. 


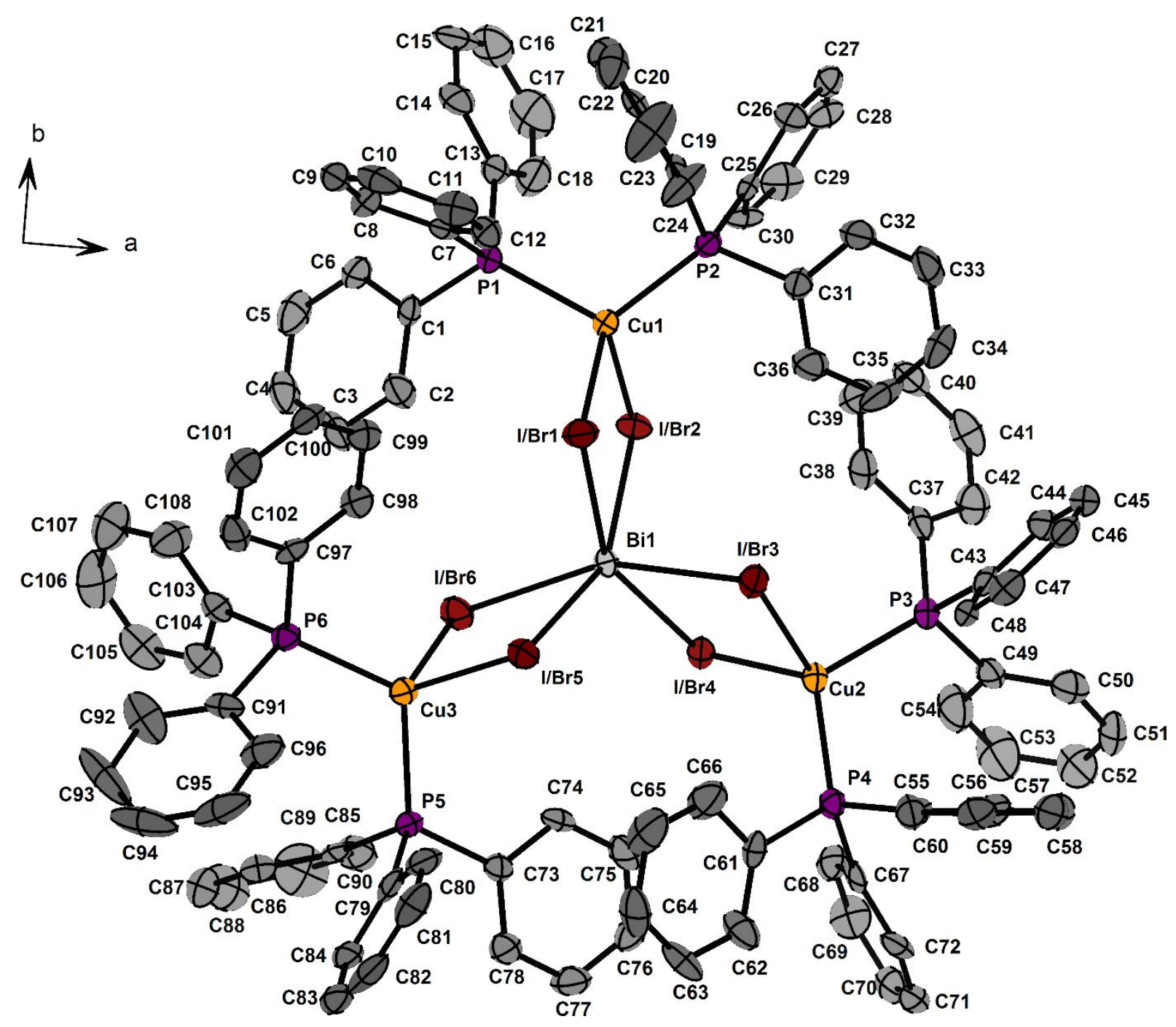

Figure S6: Asymmetric unit of 6, ellipsoids at $80 \%$ probability. 
Table S7: Crystallographic data for 7.

\begin{tabular}{|c|c|}
\hline & 7 \\
\hline Empirical formula & $\mathrm{C}_{108} \mathrm{H}_{90} \mathrm{Cu}_{3} \mathrm{I}_{6} \mathrm{P}_{6} \mathrm{Sb}$ \\
\hline Formula weight $/ \mathrm{g} \cdot \mathrm{mol}^{-1}$ & 2647.38 \\
\hline Crystal color and shape & red block \\
\hline Crystal size & $0.199 \times 0.128 \times 0.110$ \\
\hline Crystal system & orthorhombic \\
\hline Space group & $P n a 2_{1}$ \\
\hline$a / \AA$ & $31.1979(13)$ \\
\hline$b / \AA$ & $17.7861(7)$ \\
\hline$c / \AA$ & $17.7201(7)$ \\
\hline$\alpha / 0$ & 90 \\
\hline$\beta /^{\circ}$ & 90 \\
\hline$\gamma /{ }^{\circ}$ & 90 \\
\hline$V / \AA^{3}$ & $9832.7(7)$ \\
\hline$Z$ & 4 \\
\hline$\rho_{\text {calc }} / \mathrm{g} \cdot \mathrm{cm}^{-3}$ & 1.788 \\
\hline$\mu\left(\mathrm{Mo}_{\mathrm{K} \alpha}\right) / \mathrm{mm}^{-1}$ & 2.941 \\
\hline measurement temp. $/ \mathrm{K}$ & 100 \\
\hline Absorption correction type & multi-scan \\
\hline Min/max transmission & $0.6666 / 0.7452$ \\
\hline $2 \Theta$ range $/{ }^{\circ}$ & $4.538-50.676$ \\
\hline No. of measured reflections & 291019 \\
\hline No. of independent reflections & 17900 \\
\hline$R($ int $)$ & 0.0732 \\
\hline No. of indep. reflections $(I>2 \sigma(I))$ & 17058 \\
\hline No. of parameters & 1117 \\
\hline$R_{1}(I>2 \sigma(I))$ & 0.0186 \\
\hline$w R_{2}$ (all data) & 0.0339 \\
\hline$S$ (all data) & 1.033 \\
\hline$\Delta \rho_{\max }, \Delta \rho_{\min } / \mathrm{e} \cdot \AA^{-3}$ & $1.16 /-0.46$ \\
\hline
\end{tabular}

Details of crystal structure measurement and refinement: Single crystal X-ray determination was performed at $100 \mathrm{~K}$ on a Bruker Quest D8 diffractometer with microfocus MoK $\alpha$ radiation and a Photon 100 (CMOS) detector. An ISOR command had to be used on one of the carbon atoms to obtain reasonable anisotropic displacement parameters. 


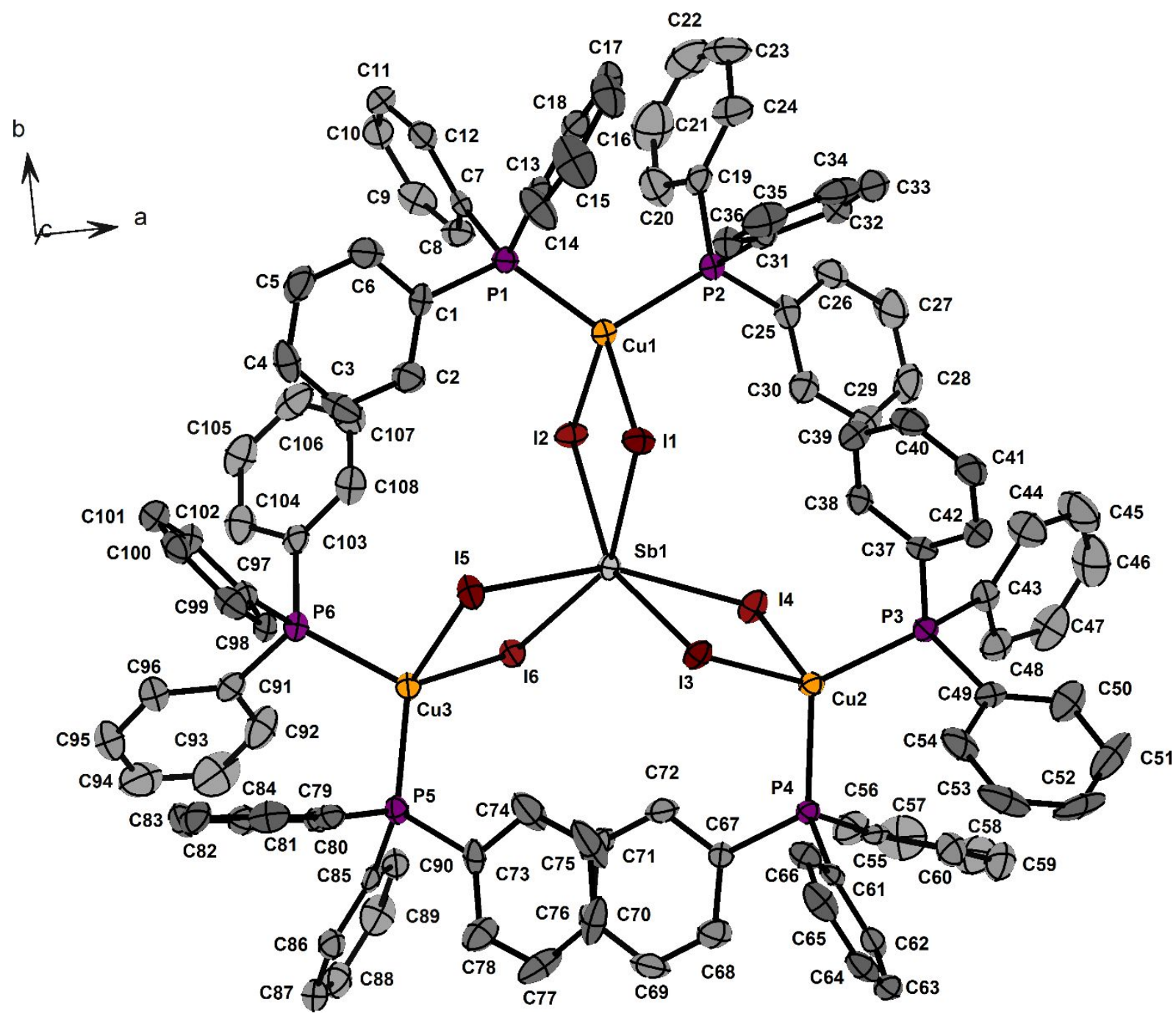

Figure S7: Asymmetric unit of 7, ellipsoids at $80 \%$ probability. 
Table S8: Crystallographic data for 8a.

\begin{tabular}{|c|c|}
\hline & $\mathbf{8 a}$ \\
\hline Empirical formula & $\mathrm{C}_{108} \mathrm{H}_{90} \mathrm{BiCu}_{3} \mathrm{I}_{6} \mathrm{P}_{6}$ \\
\hline Formula weight $/ \mathrm{g} \cdot \mathrm{mol}^{-1}$ & 2734.61 \\
\hline Crystal color and shape & red block \\
\hline Crystal size & $0.126 \times 0.084 \times 0.053$ \\
\hline Crystal system & orthorhombic \\
\hline Space group & $\operatorname{Pna}_{1}$ \\
\hline$a / \AA$ & $31.2337(12)$ \\
\hline$b / \AA$ & $17.8287(7)$ \\
\hline$c / \AA$ & $17.7415(7)$ \\
\hline$\alpha / 0$ & 90 \\
\hline$\beta /{ }^{\circ}$ & 90 \\
\hline$\gamma /{ }^{\circ}$ & 90 \\
\hline$V / \AA^{3}$ & $9879.5(7)$ \\
\hline$Z$ & 4 \\
\hline$\rho_{\text {calc }} / \mathrm{g} \cdot \mathrm{cm}^{-3}$ & 1.839 \\
\hline$\mu\left(\mathrm{Mo}_{\mathrm{K} \alpha}\right) / \mathrm{mm}^{-1}$ & 4.433 \\
\hline measurement temp. /K & 100 \\
\hline Absorption correction type & multi-scan \\
\hline Min/max transmission & $0.6451 / 0.7319$ \\
\hline $2 \Theta$ range $/{ }^{\circ}$ & $4.158-50.648$ \\
\hline No. of measured reflections & 327619 \\
\hline No. of independent reflections & 17956 \\
\hline$R($ int $)$ & 0.1045 \\
\hline No. of indep. reflections $(I>2 \sigma(I))$ & 16650 \\
\hline No. of parameters & 1117 \\
\hline$R_{1}(I>2 \sigma(I))$ & 0.0276 \\
\hline$w R_{2}$ (all data) & 0.0481 \\
\hline$S$ (all data) & 1.117 \\
\hline$\Delta \rho_{\max }, \Delta \rho_{\min } / \mathrm{e}^{-} \AA^{-3}$ & $0.74 /-0.68$ \\
\hline
\end{tabular}

Details of crystal structure measurement and refinement: Single crystal X-ray determination was performed at $100 \mathrm{~K}$ on a Bruker Quest D8 diffractometer with microfocus MoK $\alpha$ radiation and a Photon 100 (CMOS) detector. An ISOR command had to be used on eight of the carbon atoms to obtain reasonable anisotropic displacement parameters. 


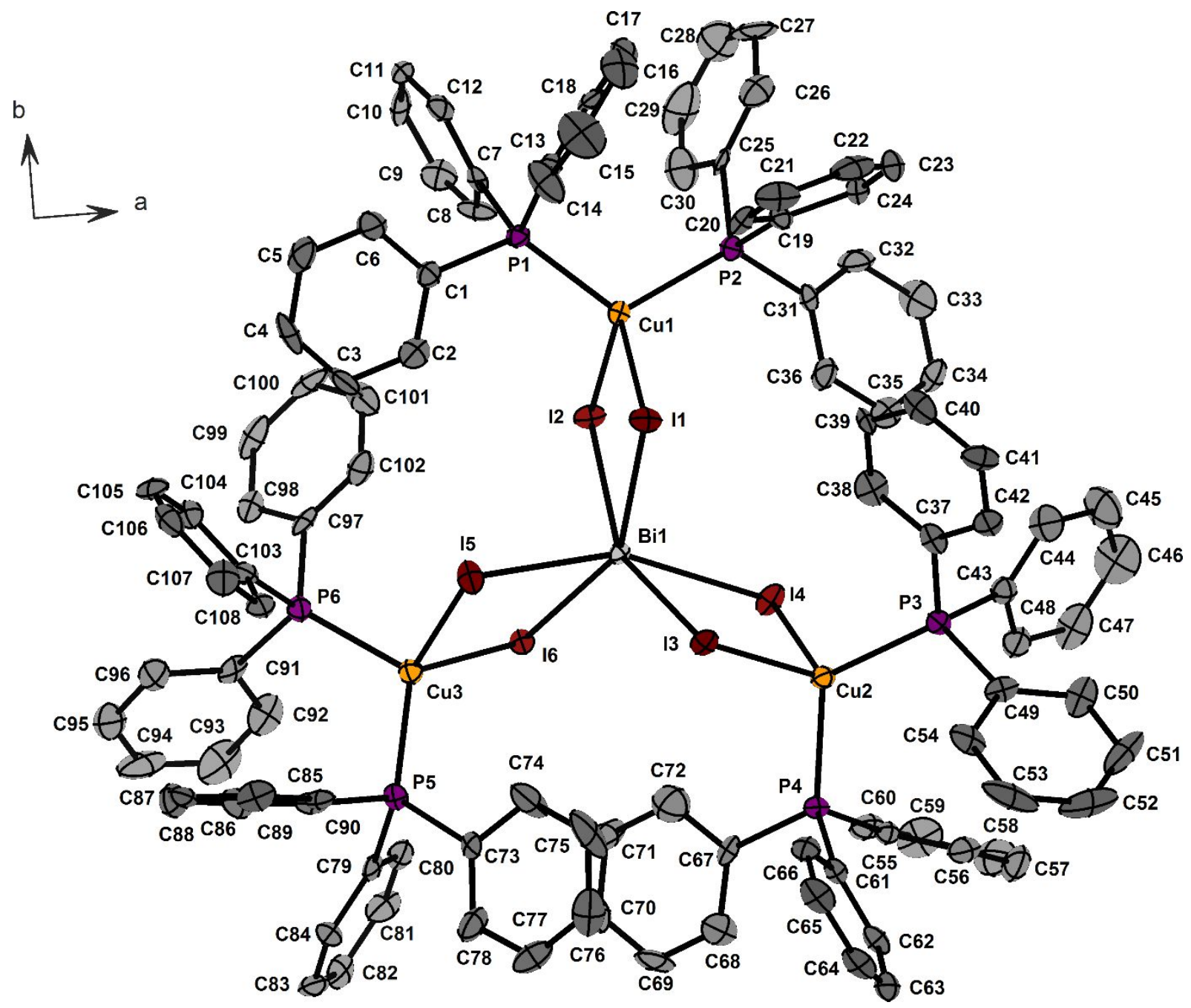

Figure S8: Asymmetric unit of $\mathbf{8 a}$, ellipsoids at $80 \%$ probability. 
Table S9: Crystallographic data for $\mathbf{8 b}$.

\begin{tabular}{|c|c|}
\hline & $\mathbf{8 b}$ \\
\hline Empirical formula & $\mathrm{C}_{116} \mathrm{H}_{99} \mathrm{BiCu}_{3} \mathrm{I}_{6} \mathrm{P}_{6}$ \\
\hline Formula weight $/ \mathrm{g} \cdot \mathrm{mol}^{-1}$ & 2734.64 \\
\hline Crystal color and shape & red block \\
\hline Crystal size & $0.121 \times 0.073 \times 0.070$ \\
\hline Crystal system & trigonal \\
\hline Space group & $R 3 c$ \\
\hline$a / \AA$ & $19.2752(4)$ \\
\hline$b / \AA$ & $19.2752(4)$ \\
\hline$c / \AA$ & $50.2974(17)$ \\
\hline$\alpha / 0$ & 90 \\
\hline$\beta /{ }^{\circ}$ & 90 \\
\hline$\gamma /{ }^{\circ}$ & 120 \\
\hline$V / \AA^{3}$ & $16183.5(9)$ \\
\hline$Z$ & 6 \\
\hline$\rho_{\text {calc }} / \mathrm{g} \cdot \mathrm{cm}^{-3}$ & 1.783 \\
\hline$\mu\left(\mathrm{Mo}_{\mathrm{K} \alpha}\right) / \mathrm{mm}^{-1}$ & 18.514 \\
\hline measurement temp. /K & 100 \\
\hline Absorption correction type & multi-scan \\
\hline Min/max transmission & $0.0086 / 0.0490$ \\
\hline $2 \Theta$ range $/{ }^{\circ}$ & $6.356-136$ \\
\hline No. of measured reflections & 33819 \\
\hline No. of independent reflections & 5931 \\
\hline$R($ int $)$ & 0.0686 \\
\hline No. of indep. reflections $(I>2 \sigma(I))$ & 4787 \\
\hline No. of parameters & 351 \\
\hline$R_{1}(I>2 \sigma(I))$ & 0.0438 \\
\hline$w R_{2}$ (all data) & 0.0997 \\
\hline$S$ (all data) & 0.923 \\
\hline$\Delta \rho_{\max }, \Delta \rho_{\min } / \mathrm{e}^{-} \AA^{-3}$ & $1.32 /-1.70$ \\
\hline
\end{tabular}

Details of crystal structure measurement and refinement: Single crystal X-ray determination was performed at $100 \mathrm{~K}$ on a STOE StadiVari diffractometer using $\mathrm{Cu} \mathrm{K} \alpha$ radiation from an $\mathrm{X}$-ray micro source with X-ray optics and a Pilatus 300K Si hybrid pixel array detector. An ISOR command had to be used on three of the carbon atoms to obtain reasonable anisotropic displacement parameters. A total of four disordered molecules of acetonitrile per formula unit on two positions can be located. One molecule is disordered about the threefold axis in such a way that an occupancy of $1 / 3$ has to be chosen for the nitrogen and the carbon atoms. For this position, hydrogen atoms could not be included. On the second position acetonitrile molecules are disordered across two positions with occupancies of 0.5. Disordered atoms were refined isotropically. 


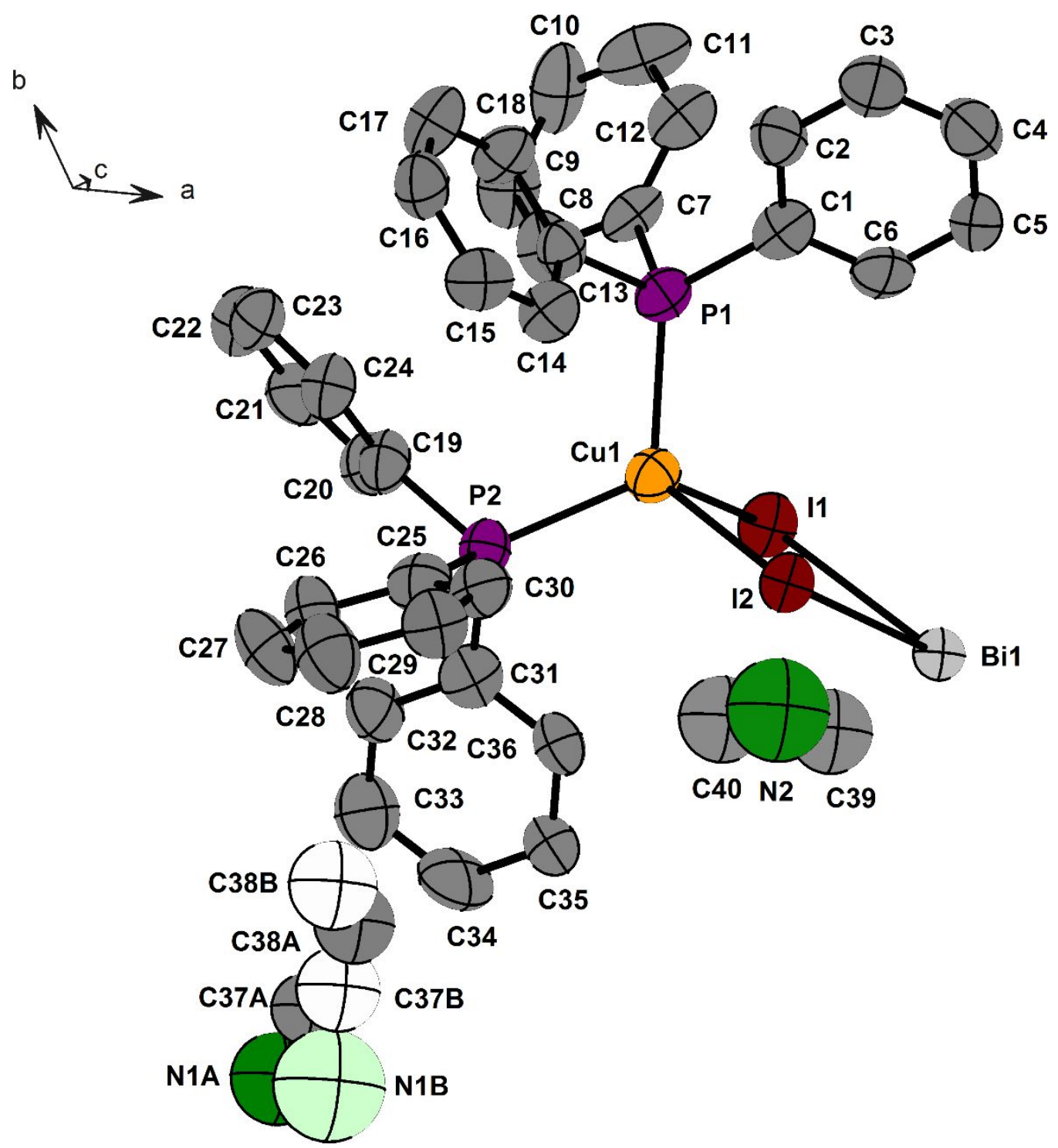

Figure 59: Asymmetric unit of $\mathbf{8 b}$, ellipsoids at $80 \%$ probability. 
Table S10: Crystallographic data for $9 \mathrm{~b}$.

\begin{tabular}{|c|c|}
\hline & $9 \mathbf{b}$ \\
\hline Empirical formula & $\mathrm{C}_{116} \mathrm{H}_{99} \mathrm{Ag}_{3} \mathrm{BiI}_{6} \mathrm{~N}_{4} \mathrm{P}_{6}$ \\
\hline Formula weight $/ \mathrm{g} \cdot \mathrm{mol}^{-1}$ & 3028.83 \\
\hline Crystal color and shape & red block \\
\hline Crystal size & $0.099 \times 0.099 \times 0.073$ \\
\hline Crystal system & trigonal \\
\hline Space group & $R 3 c$ \\
\hline$a / \AA$ & $19.6386(8)$ \\
\hline$b / \AA$ & $19.6386(8)$ \\
\hline$c / \AA$ & $49.5036(19)$ \\
\hline$\alpha /{ }^{\circ}$ & 90 \\
\hline$\beta /^{\circ}$ & 90 \\
\hline$\gamma /{ }^{\circ}$ & 120 \\
\hline$V / \AA^{3}$ & $16534.4(15)$ \\
\hline$Z$ & 6 \\
\hline$\rho_{\text {calc }} / \mathrm{g} \cdot \mathrm{cm}^{-3}$ & 1.825 \\
\hline$\mu\left(\mathrm{Mo}_{\mathrm{K} \alpha}\right) / \mathrm{mm}^{-1}$ & 3.933 \\
\hline measurement temp. $/ \mathrm{K}$ & 100 \\
\hline Absorption correction type & multi-scan \\
\hline Min/max transmission & $0.6319 / 0.7452$ \\
\hline $2 \Theta$ range $/{ }^{\circ}$ & $4.828-50.66$ \\
\hline No. of measured reflections & 35570 \\
\hline No. of independent reflections & 6635 \\
\hline$R$ (int) & 0.0834 \\
\hline No. of indep. reflections $(I>2 \sigma(I))$ & 5860 \\
\hline No. of parameters & 407 \\
\hline$R_{1}(I>2 \sigma(I))$ & 0.0344 \\
\hline$w R_{2}$ (all data) & 0.0642 \\
\hline$S$ (all data) & 0.988 \\
\hline$\Delta \rho_{\max }, \Delta \rho_{\min } / \mathrm{e} \cdot \AA^{-3}$ & $1.66 /-0.72$ \\
\hline
\end{tabular}

Details of crystal structure measurement and refinement: Single crystal X-ray determination was performed at $100 \mathrm{~K}$ on a Bruker Quest D8 diffractometer with microfocus MoK $\alpha$ radiation and a Photon 100 (CMOS) detector. A total of four disordered molecules of acetonitrile can be located. For the molecule disordered about the threefold axis, an occupancy of $1 / 3$ had to be chosen for the nitrogen atom and no reasonable hydrogen atom positions could be included. A second position of acetonitrile molecules features partial disorder in the $\mathrm{C} \equiv \mathrm{N}$ group with occupancies of 0.5 . Disordered atoms were refined isotropically. 
b

$\sum x^{c}>a$
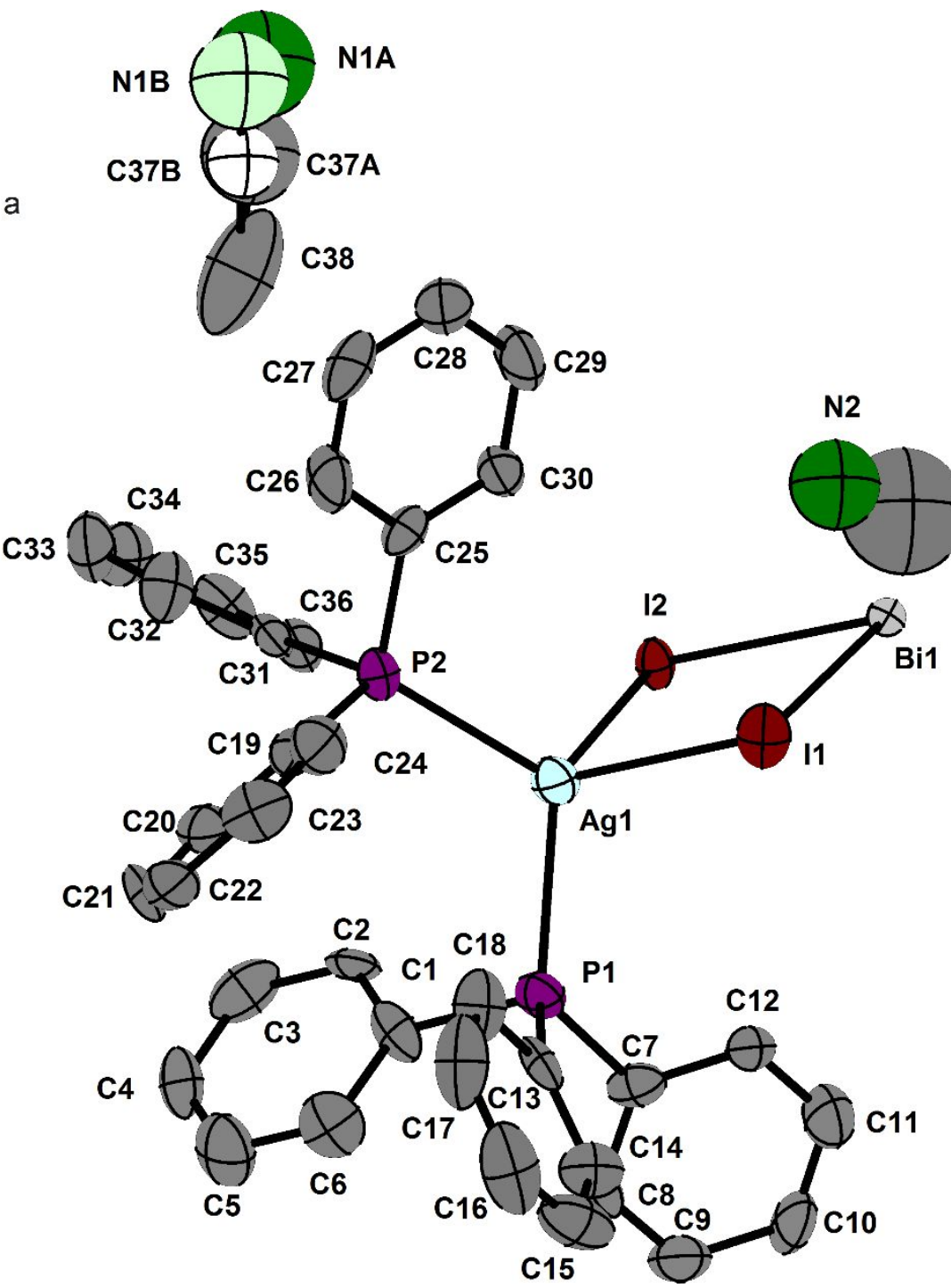

Figure S10: Asymmetric unit of 9b, ellipsoids at $80 \%$ probability. 
Table S11: Selected bond length in Angstroms for compounds 1, 2, 3a.

\begin{tabular}{|c|c|c|c|}
\hline & $\begin{array}{c}{\left[\mathrm{Bi}_{2} \mathrm{Cu}_{2} \mathrm{Cl}_{5}\left(\mathrm{PPh}_{3}\right)_{4}\left(\mathrm{Me}_{2} \mathrm{CO}\right)\right]} \\
\cdot 2 \mathrm{Me}_{2} \mathrm{CO}(\mathbf{1})\end{array}$ & $\begin{array}{c}{\left[\mathrm{BiAg}_{2} \mathrm{Cl}_{5}\left(\mathrm{PPh}_{3}\right)_{4}\left(\mathrm{Me}_{2} \mathrm{CO}\right)\right]} \\
(\mathbf{2})\end{array}$ & $\begin{array}{c}{\left[\mathrm{BiAg}_{2} \mathrm{Br}_{5}\left(\mathrm{PPh}_{3}\right)_{4}(\mathrm{MeCN})\right]} \\
(\mathbf{3 a})\end{array}$ \\
\hline $\mathbf{B i}-\mathbf{L}$ & 2.597 & 2.747 & 2.789 \\
\hline $\mathbf{B i}-\mathbf{X}$ & $2.490-3.050$ & $2.497-2.754$ & $2.831-2.858$ \\
\hline $\mathbf{M}-\mathbf{X}$ & $2.335-2.798$ & $2.620-2.879$ & $2.715-2.956$ \\
\hline $\mathbf{M}-\mathbf{P}$ & $2.270-2.272$ & $2.454-2.462$ & $2.452-2.458$ \\
\hline
\end{tabular}

Table S12: Selected bond length in Angstroms for compounds 4-6, data for 5a from the CCDC dataset QUKVIC ${ }^{4}$ twist angle $\phi$ in ${ }^{\circ}$ between triangles formed by halogen ligands orthogonal to the threefold axis. ${ }^{5}$

\begin{tabular}{|c|c|c|c|c|}
\hline & $\begin{array}{c}{\left[\mathrm{BiCu}_{3} \mathrm{Br}_{6}\left(\mathrm{PPh}_{3}\right)_{6}\right]} \\
\text { (4) }\end{array}$ & $\begin{array}{c}{\left[\mathrm{BiAg}_{3} \mathrm{Br}_{6}\left(\mathrm{PPh}_{3}\right)_{6}\right]} \\
(\mathbf{5 a})\end{array}$ & $\begin{array}{c}{\left[\mathrm{BiAg}_{3} \mathrm{Br}_{6}\left(\mathrm{PPh}_{3}\right)_{6}\right]} \\
\cdot 4 \mathrm{MeCN}(\mathbf{5 b})\end{array}$ & $\begin{array}{c}{\left[\mathrm{BiCu}_{3} \mathrm{I}_{3} \mathrm{Br}_{3}\left(\mathrm{PPh}_{3}\right)_{6}\right]} \\
(6)\end{array}$ \\
\hline $\mathrm{Bi}-\mathrm{X}$ & $2.799-2.949$ & $2.805-2.940$ & $2.859-2.891$ & $2.867-3.089$ \\
\hline$M-X$ & $2.513-2.604$ & $2.693-2.820$ & $2.780-2.797$ & $2.610-2.666$ \\
\hline M-P & $2.263-2.273$ & $2.448-2.475$ & $2.474-2.478$ & $2.269-2.281$ \\
\hline$\phi$ & 30 & 40 & 42 & 29 \\
\hline
\end{tabular}

Table S13: Selected bond length in Angstroms for compounds 7-9b and twist angle $\phi$ in ${ }^{\circ}$ between triangles formed by halogen ligands orthogonal to the threefold axis. ${ }^{5}$ Data for $8 \mathrm{a}$ is similar to that found by Pike and coworkers (CCDC dataset CIPWIK). ${ }^{6}$

\begin{tabular}{|c|c|c|c|c|}
\hline & $\begin{array}{c}{\left[\mathrm{SbCu}_{3} \mathrm{I}_{6}\left(\mathrm{PPh}_{3}\right)_{6}\right]} \\
\mathbf{( 7 )}\end{array}$ & $\begin{array}{c}{\left[\mathrm{BiCu}_{3} \mathrm{I}_{6}\left(\mathrm{PPh}_{3}\right)_{6}\right]} \\
(\mathbf{8 a})\end{array}$ & $\begin{array}{c}{\left[\mathrm{BiCu} \mathrm{I}_{6}\left(\mathrm{PPh}_{3}\right)_{6}\right] \cdot} \\
4 \mathrm{MeCN}(\mathbf{8 b})\end{array}$ & $\begin{array}{c}{\left[\mathrm{BiAg} 3 \mathrm{I}_{6}\left(\mathrm{PPh}_{3}\right)_{6}\right] \cdot} \\
4 \mathrm{MeCN}(\mathbf{9 b})\end{array}$ \\
\hline $\mathbf{E}-\mathbf{X}$ & $2.934-3.153$ & $3.012-3.177$ & $3.105-3.113$ & $3.090-3.091$ \\
\hline $\mathbf{M}-\mathbf{X}$ & $2.654-2.742$ & $2.665-2.727$ & $2.696-2.728$ & $2.883-2.918$ \\
\hline $\mathbf{M}-\mathbf{P}$ & $2.271-2.286$ & $2.271-2.284$ & $2.288-2.294$ & $2.480-2.487$ \\
\hline $\mathbf{\phi}$ & 30 & 28 & 28 & 39 \\
\hline
\end{tabular}




\section{Thermal analysis}

The thermal behavior of 2 (14.9 mg), was studied by TGA/DSC on a NETSCH STA 409 C/CD from $25^{\circ} \mathrm{C}$ to $1000{ }^{\circ} \mathrm{C}$ with a heating rate of $10^{\circ} \mathrm{C} \mathrm{min}^{-1}$ in a constant flow of $80 \mathrm{ml} \mathrm{min}^{-1} \mathrm{~N}_{2}$.

A first mass loss of $2.3 \%$ is observed starting at $120^{\circ} \mathrm{C}$, corresponding to the loss of 0.7 molecules of acetone per formula unit. A second mass loss starts at $240{ }^{\circ} \mathrm{C}$ and corresponds to the decomposition of the compound, possibly involving the loss of $\mathrm{PPh}_{3}$ and $\mathrm{BiCl}_{3}$. The endothermic peak at $242.2^{\circ} \mathrm{C}$ likely corresponds to a melting point concomitant with the onset of decomposition.

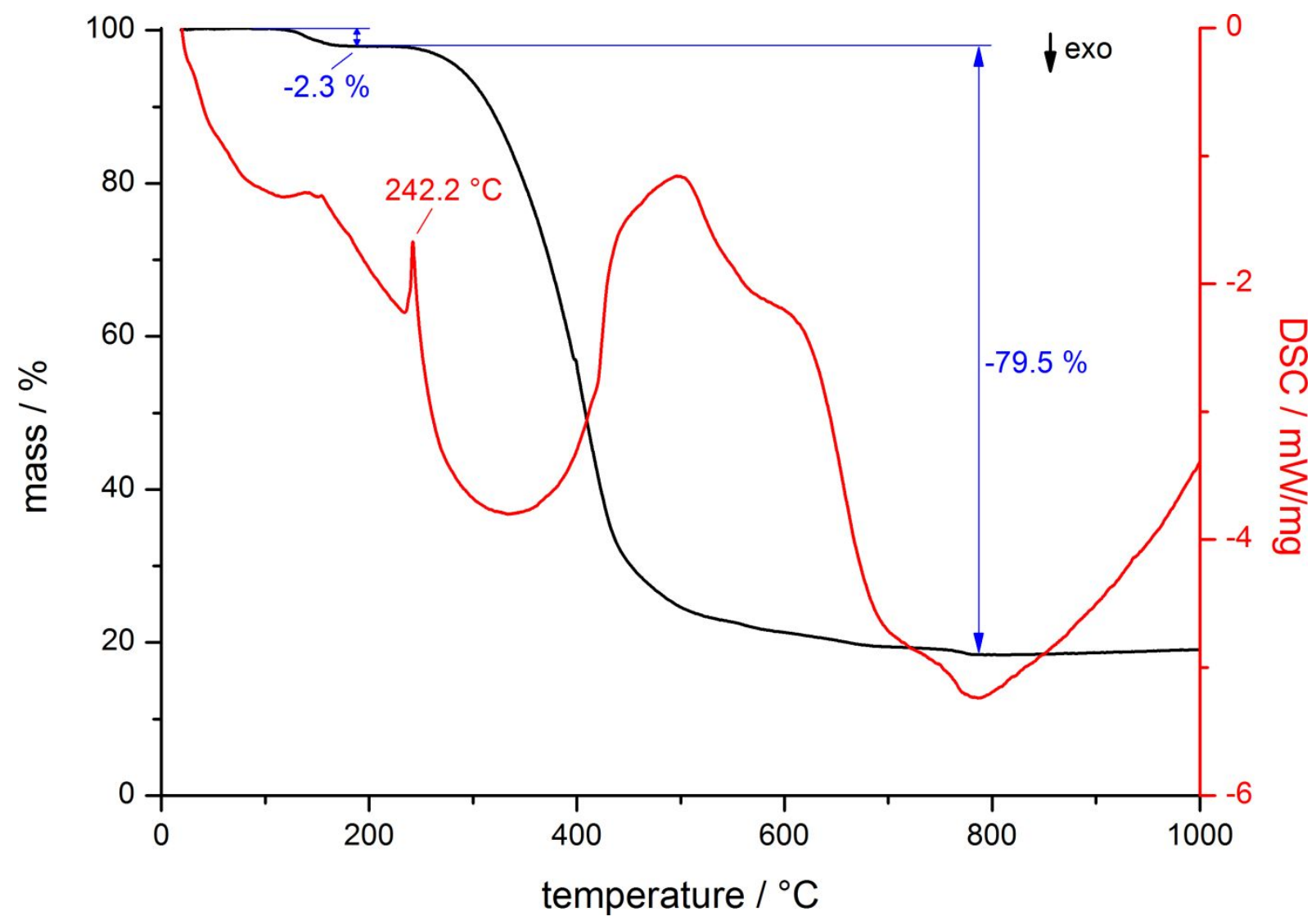

Figure S11. TGA/DSC of 2. 
The thermal behavior of $3 a(17.0 \mathrm{mg})$, was studied by TGA/DSC on a NETSCH STA $409 \mathrm{C} / \mathrm{CD}$ from $25^{\circ} \mathrm{C}$ to $1000^{\circ} \mathrm{C}$ with a heating rate of $10^{\circ} \mathrm{C} \mathrm{min}^{-1}$ in a constant flow of $80 \mathrm{ml} \mathrm{min}-1 \mathrm{~N}_{2}$.

A first mass loss of $1.5 \%$ is observed starting at $110{ }^{\circ} \mathrm{C}$, corresponding to the loss of 0.7 molecules of acetonitrile per formula unit. A second mass loss starts at $230^{\circ} \mathrm{C}$ and corresponds to the decomposition of the compound, possibly involving the loss of $\mathrm{PPh}_{3}$ and $\mathrm{BiBr}_{3}$. The endothermic peak at $232.4^{\circ} \mathrm{C}$ likely corresponds to a melting point concomitant with the onset of decomposition, while the peak at $959.3^{\circ} \mathrm{C}$ can be attributed to the melting point of silver.

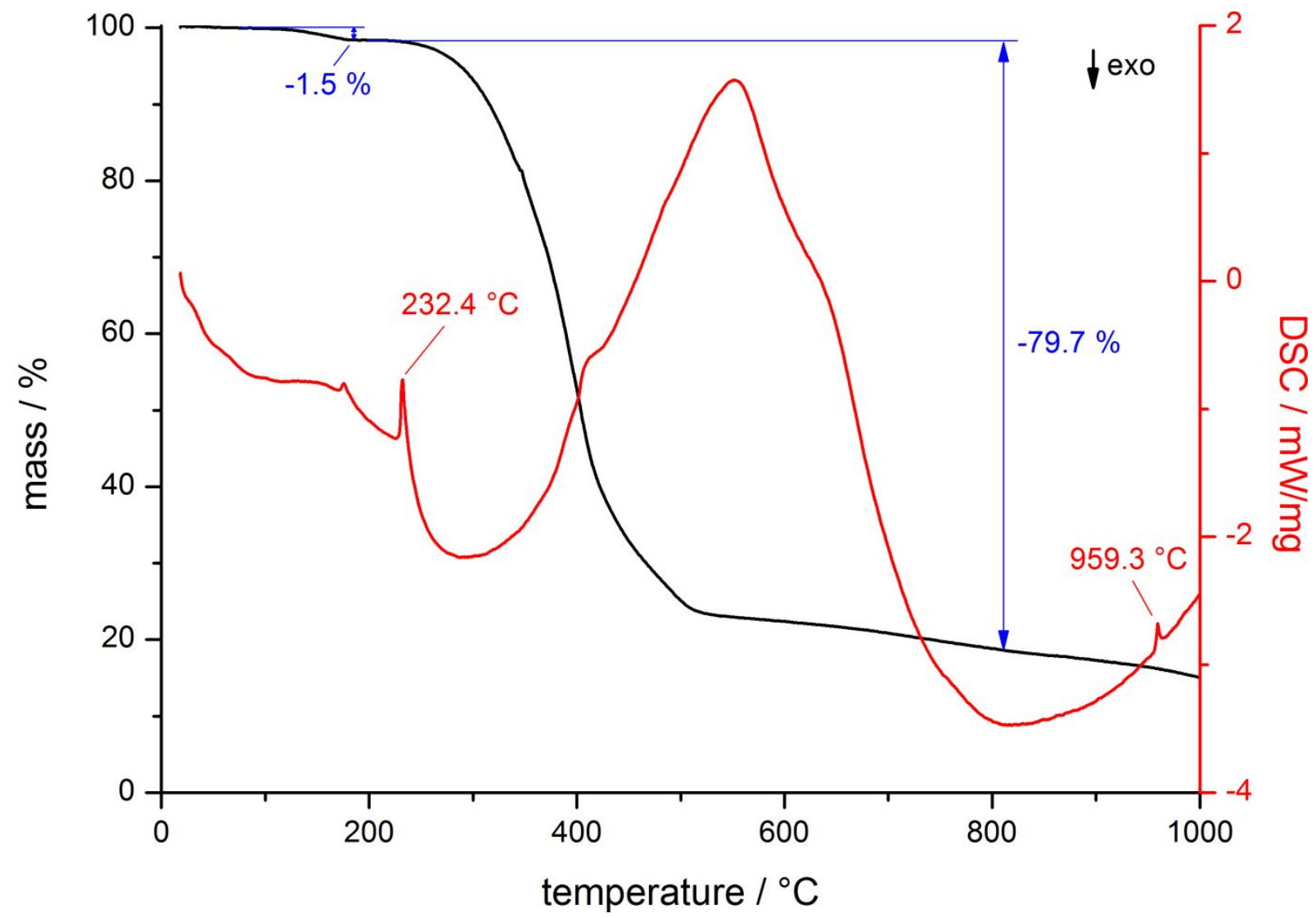

Figure S12. TGA/DSC of 3a. 
The thermal behavior of $\mathbf{3 b}(22.5 \mathrm{mg})$, was studied by TGA/DSC on a NETSCH STA $409 \mathrm{C} / \mathrm{CD}$ from $25^{\circ} \mathrm{C}$ to $1000^{\circ} \mathrm{C}$ with a heating rate of $10^{\circ} \mathrm{C} \mathrm{min}^{-1}$ in a constant flow of $80 \mathrm{ml} \mathrm{min}-1 \mathrm{~N}_{2}$.

A first mass loss of $2.3 \%$ is observed starting at $115^{\circ} \mathrm{C}$, corresponding to the loss of 0.7 molecules of acetone per formula unit. A second mass loss starts at $230{ }^{\circ} \mathrm{C}$ and corresponds to the decomposition of the compound, possibly involving the loss of $\mathrm{PPh}_{3}$ and $\mathrm{BiBr}_{3}$. The strikingly similar position of the first major endothermic peak at $232{ }^{\circ} \mathrm{C}$, likely a melting point shortly before the onset of decomposition, in both $\mathbf{3 a}$ and $\mathbf{3 b}$ suggests that both compounds are identical after the loss of coordinating solvent.

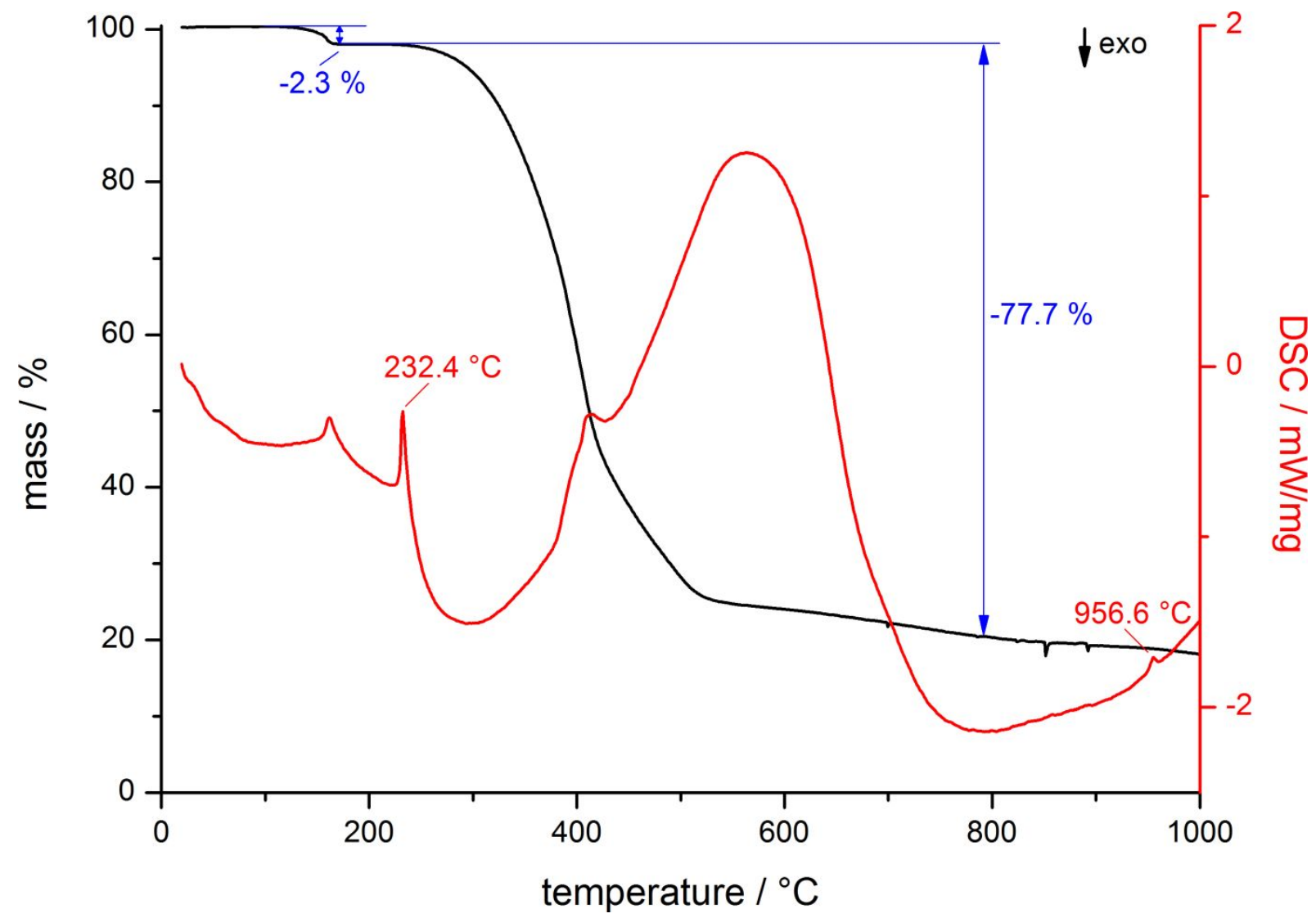

Figure S13. TGA/DSC of $\mathbf{3 b}$. 
The thermal behavior of 4 (16.1 mg), was studied by TGA/DSC on a NETSCH STA $409 \mathrm{C} / \mathrm{CD}$ from $25^{\circ} \mathrm{C}$ to $1000{ }^{\circ} \mathrm{C}$ with a heating rate of $10{ }^{\circ} \mathrm{C} \mathrm{min}^{-1}$ in a constant flow of $80 \mathrm{ml} \mathrm{min}^{-1} \mathrm{~N}_{2}$.

A single multi-step mass loss is observed starting at $230^{\circ} \mathrm{C}$ corresponding to the decomposition of the compound, possibly involving the loss of $\mathrm{PPh}_{3}$ and $\mathrm{BiBr}_{3}$. The endothermic peak at $203.3^{\circ} \mathrm{C}$ corresponds to a melting point concomitant with the onset of decomposition.

To verify the nature of this phase transition, we performed a melting point determination on a KSP I $N$ melting point meter (A. Krüss Optronic, Germany) and found a melting point of $202.3^{\circ} \mathrm{C}$.

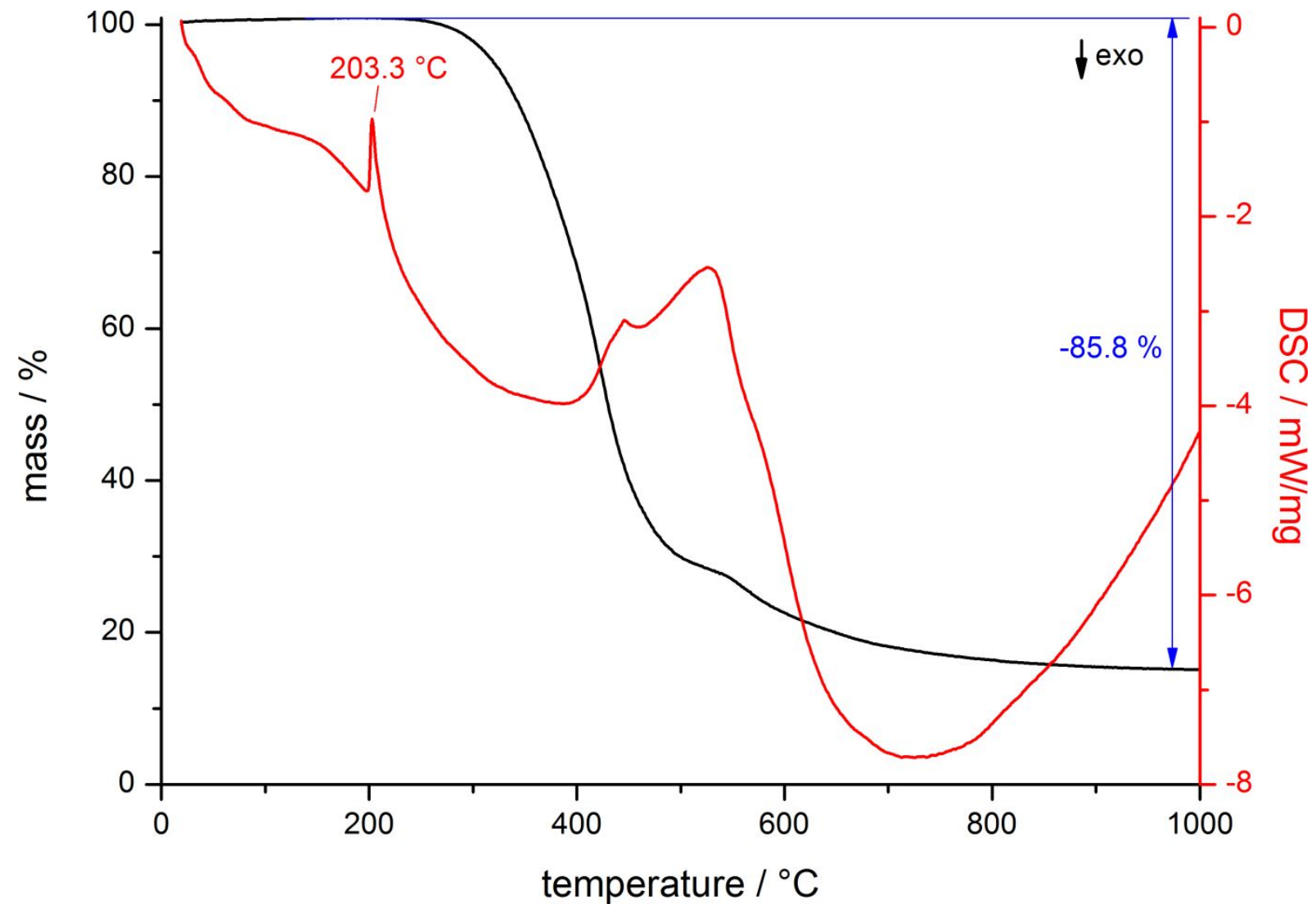

Figure S14. TGA/DSC of 4. 
The thermal behavior of $5 \mathrm{a}(14.3 \mathrm{mg})$, was studied by TGA/DSC on a NETSCH STA $409 \mathrm{C} / \mathrm{CD}$ from $25^{\circ} \mathrm{C}$ to $1000^{\circ} \mathrm{C}$ with a heating rate of $10^{\circ} \mathrm{C} \mathrm{min}^{-1}$ in a constant flow of $80 \mathrm{ml} \mathrm{min} \mathrm{m}^{-1} \mathrm{~N}_{2}$.

A single multi-step mass loss is observed starting at $220^{\circ} \mathrm{C}$ corresponding to the decomposition of the compound, possibly involving the loss of $\mathrm{PPh}_{3}$ and $\mathrm{BiBr}_{3}$. In comparison with the TG data reported by Fenske, ${ }^{3}$ we observe a ca. $70^{\circ} \mathrm{C}$ higher onset of decomposition, which we attribute to the difference in experimental conditions: The literature data was recorded under vacuum with a heating rate of $2{ }^{\circ} \mathrm{C} \mathrm{min}^{-1}$, while we performed our measurements under a flow of nitrogen with a heating rate of $10^{\circ} \mathrm{C} \mathrm{min}-1$.

The endothermic peak at $210.8^{\circ} \mathrm{C}$ corresponds to a melting point concomitant with the onset of decomposition, while the peak at $960.3^{\circ} \mathrm{C}$ can be attributed to the melting point of silver.

To verify the nature of the first phase transition, we performed a melting point determination on a on a KSP I N melting point meter (A. Krüss Optronic, Germany) and found a melting point of $210.8^{\circ} \mathrm{C}$.

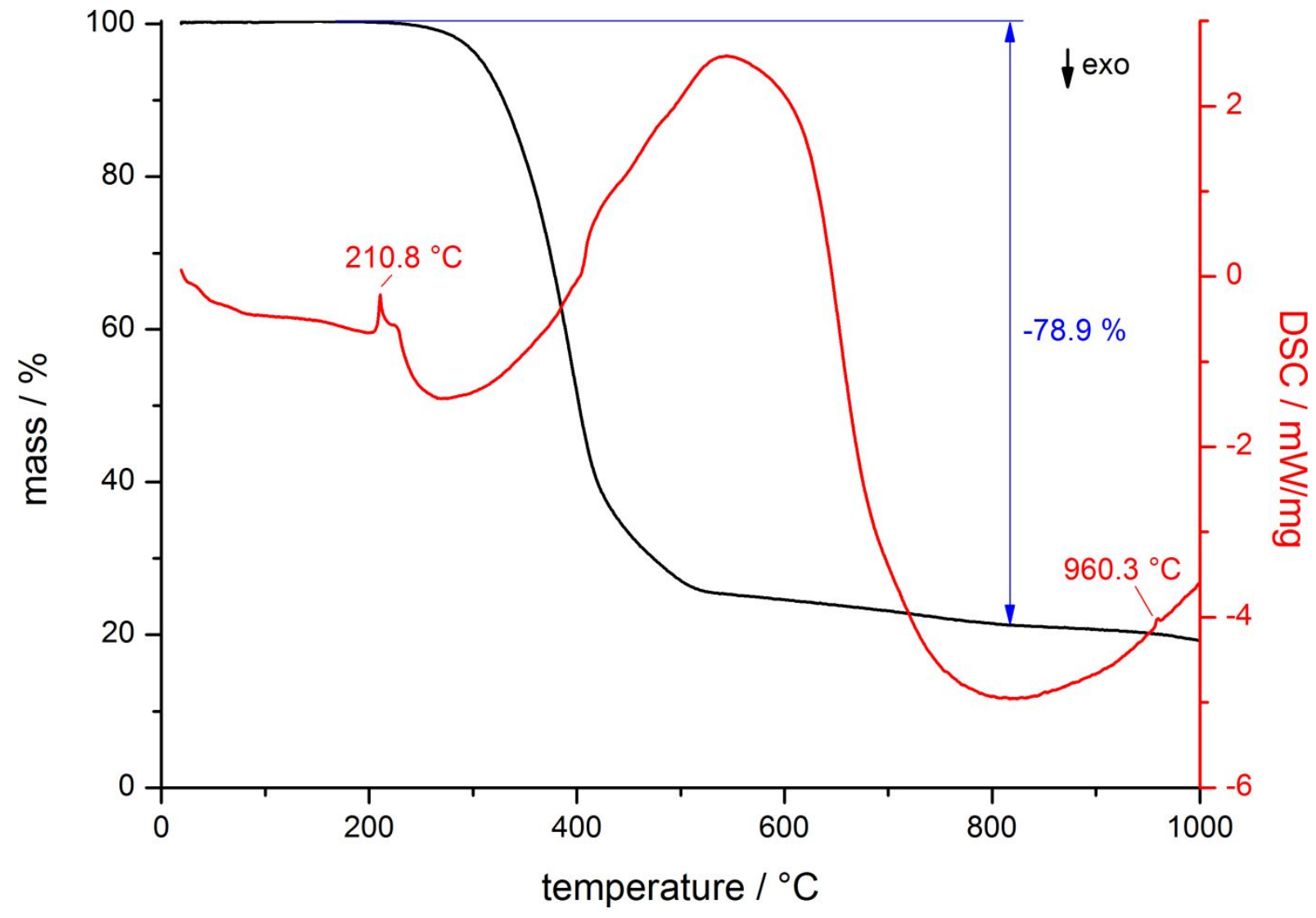

Figure S15. TGA/DSC of $\mathbf{5 a .}$ 
The thermal behavior of 6 (12.9 mg), was studied by TGA/DSC on a NETSCH STA $409 \mathrm{C} / \mathrm{CD}$ from $25^{\circ} \mathrm{C}$ to $1000{ }^{\circ} \mathrm{C}$ with a heating rate of $10^{\circ} \mathrm{C} \mathrm{min}^{-1}$ in a constant flow of $80 \mathrm{ml} \mathrm{min}^{-1} \mathrm{~N}_{2}$.

A single multi-step mass loss is observed starting at $200{ }^{\circ} \mathrm{C}$ corresponding to the decomposition of the compound, possibly involving the loss of $\mathrm{PPh}_{3}, \mathrm{BiBr}_{3}$ and $\mathrm{Bil}_{3}$. The endothermic peak at $206.5^{\circ} \mathrm{C}$ likely corresponds to a melting point concomitant with the onset of decomposition.

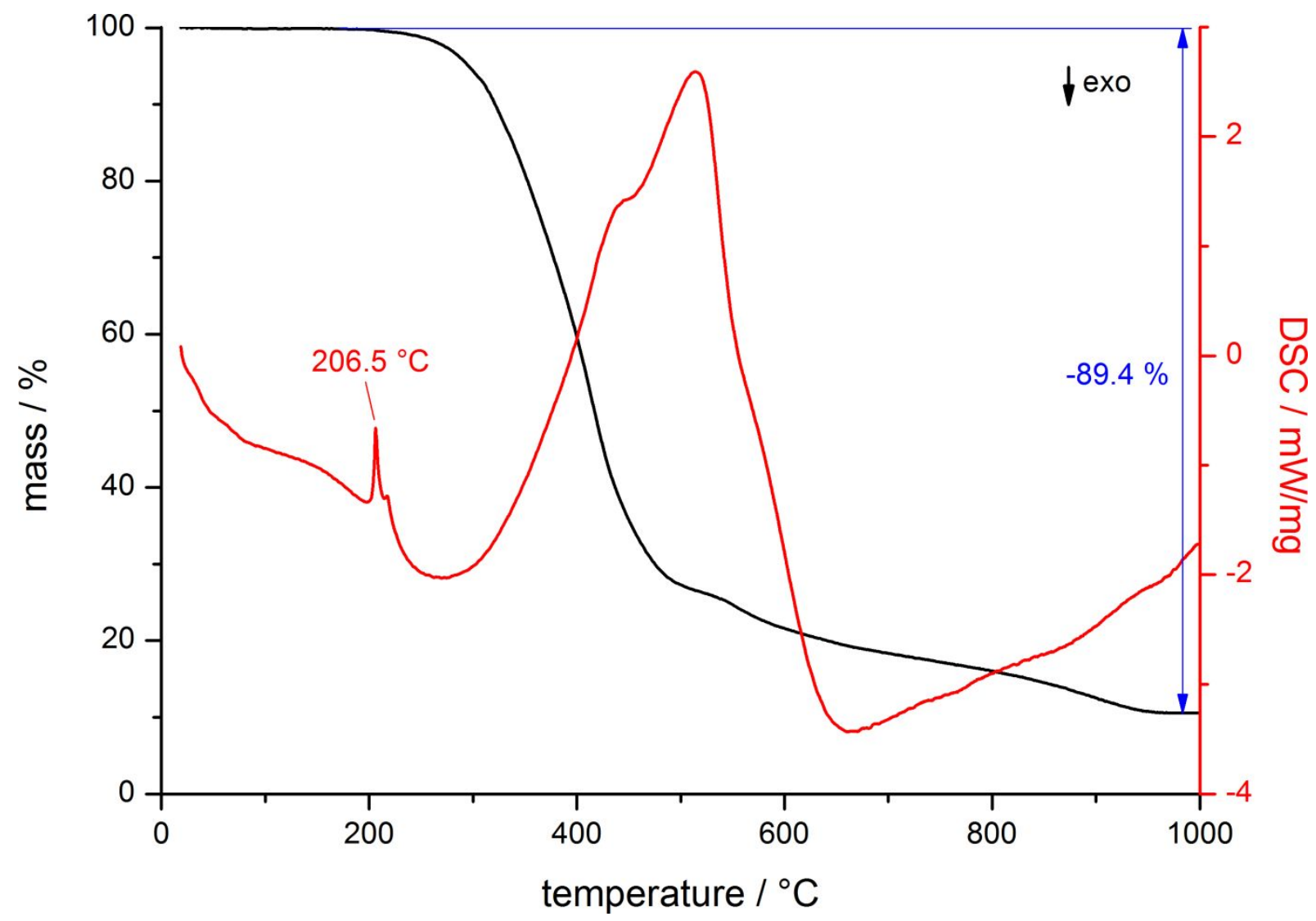

Figure S16. TGA/DSC of 6. 
The thermal behavior of 7 ( $17.8 \mathrm{mg}$ ), was studied by TGA/DSC on a NETSCH STA $409 \mathrm{C} / \mathrm{CD}$ from $25^{\circ} \mathrm{C}$ to $1000{ }^{\circ} \mathrm{C}$ with a heating rate of $10^{\circ} \mathrm{C} \mathrm{min}^{-1}$ in a constant flow of $80 \mathrm{ml} \mathrm{min}^{-1} \mathrm{~N}_{2}$.

A single mass loss step is observed starting at $185^{\circ} \mathrm{C}$ corresponding to the decomposition of the compound, possibly involving the loss of $\mathrm{PPh}_{3}$ and $\mathrm{Sbl}_{3}$. The endothermic peak at $171.4{ }^{\circ} \mathrm{C}$ likely corresponds to a melting point shortly before the onset of decomposition.

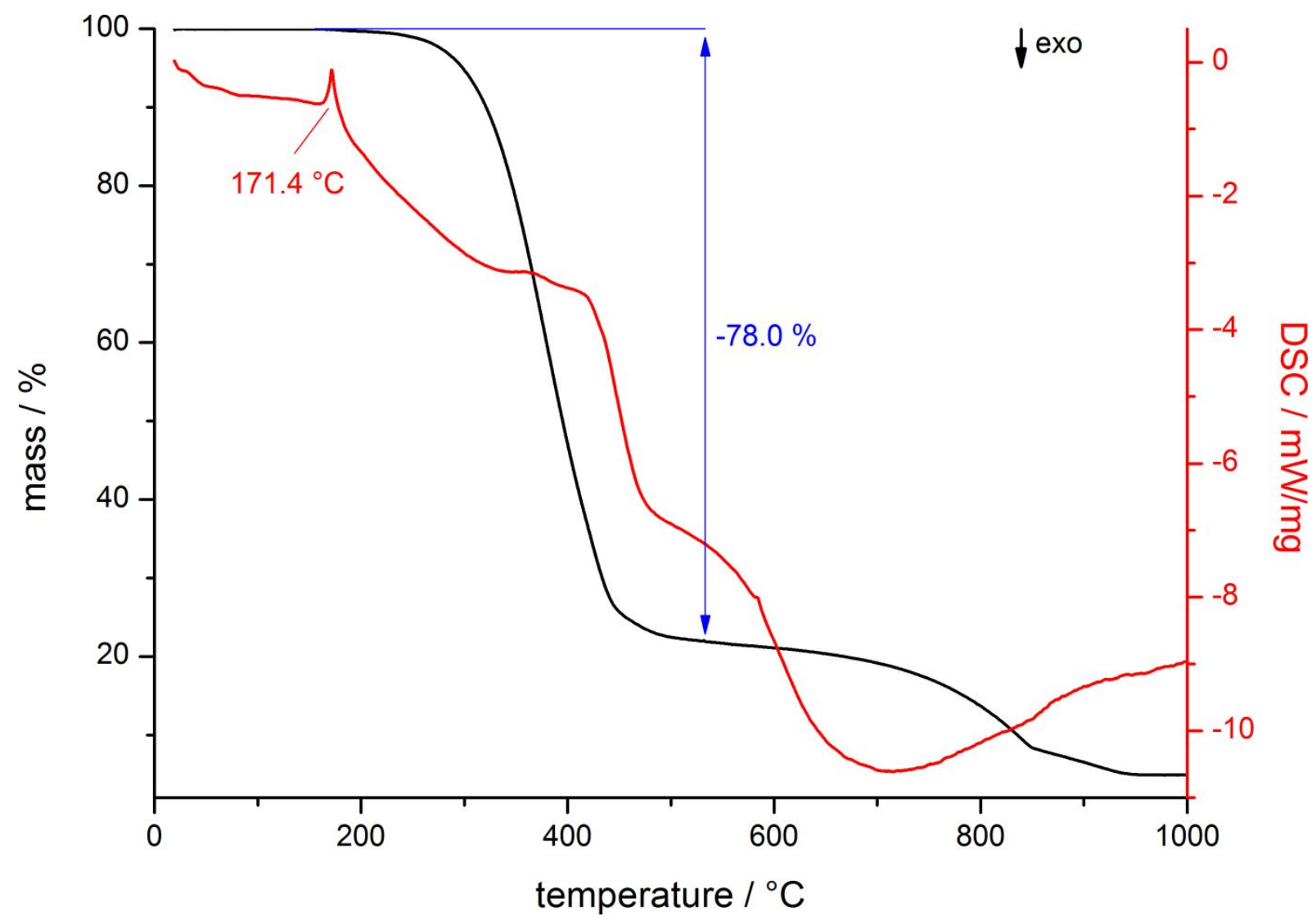

Figure S17. TGA/DSC of 7. 
The thermal behavior of $8 \mathrm{a}(15.0 \mathrm{mg})$, was studied by TGA/DSC on a NETSCH STA $409 \mathrm{C} / \mathrm{CD}$ from $25^{\circ} \mathrm{C}$ to $1000^{\circ} \mathrm{C}$ with a heating rate of $10^{\circ} \mathrm{C} \mathrm{min}^{-1}$ in a constant flow of $80 \mathrm{ml} \mathrm{min}-1 \mathrm{~N}_{2}$.

A single mass loss step is observed starting at $210^{\circ} \mathrm{C}$ corresponding to the decomposition of the compound, possibly involving the loss of $\mathrm{PPh}_{3}$ and $\mathrm{Bil}_{3}$. The endothermic peak at $213.5^{\circ} \mathrm{C}$ likely corresponds to a melting point concomitant with the onset of decomposition.

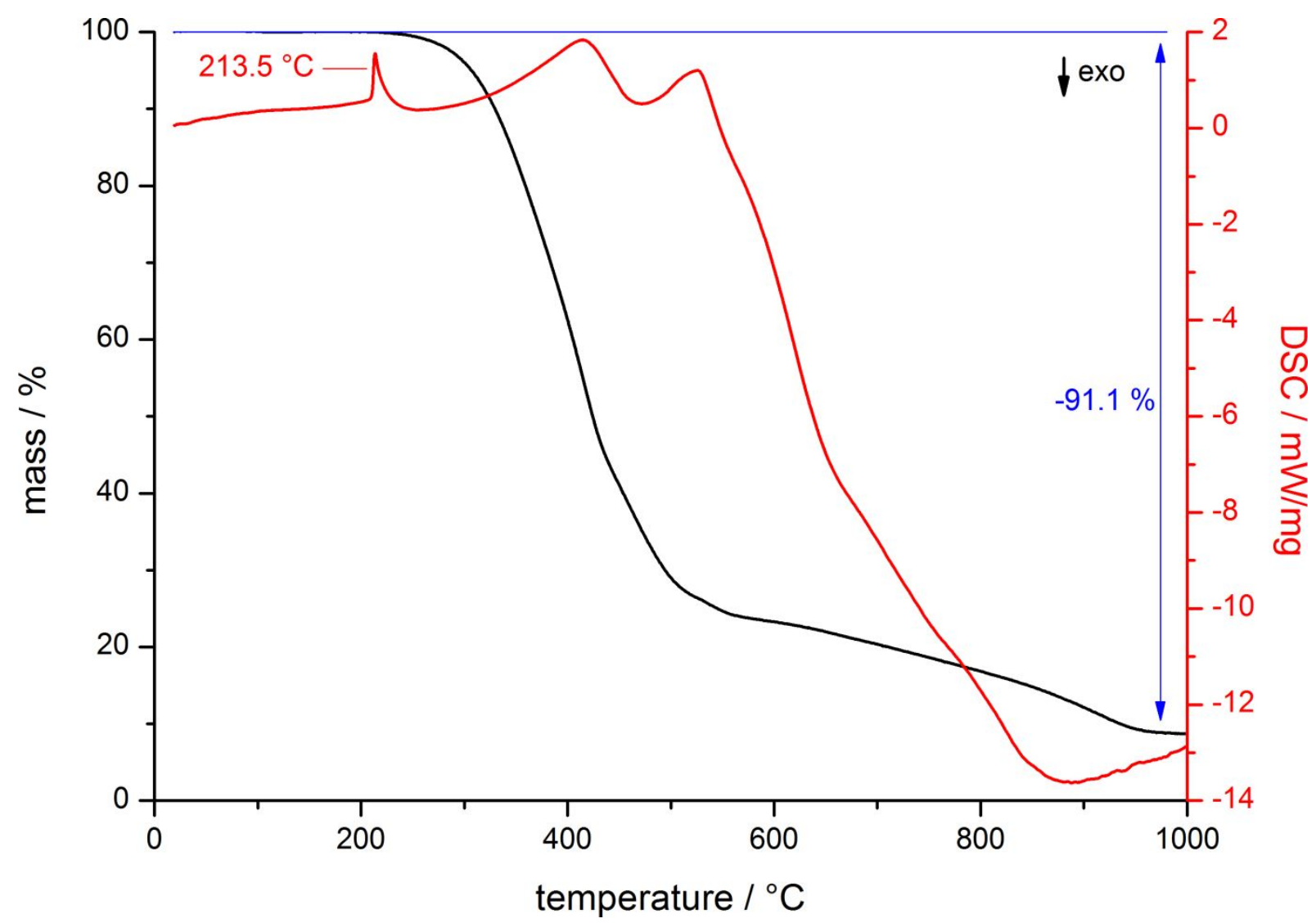

Figure S18. TGA/DSC of 8 a. 
The thermal behavior of $\mathbf{8 b}$ (19.2 $\mathrm{mg})$, was studied by TGA/DSC on a NETSCH STA $409 \mathrm{C} / \mathrm{CD}$ from $25^{\circ} \mathrm{C}$ to $1000^{\circ} \mathrm{C}$ with a heating rate of $10^{\circ} \mathrm{C} \mathrm{min}^{-1}$ in a constant flow of $80 \mathrm{ml} \mathrm{min}-1 \mathrm{~N}_{2}$.

A first mass loss of $5.6 \%$ is observed starting at $100{ }^{\circ} \mathrm{C}$, corresponding to the loss of 4 molecules of acetonitrile per formula unit. A second mass loss starts at $230^{\circ} \mathrm{C}$ and corresponds to the decomposition of the compound, possibly involving the loss of $\mathrm{PPh}_{3}$ and $\mathrm{Bil}_{3}$. The similar position of the endothermic peak at $212^{\circ} \mathrm{C}$, likely a melting point shortly before the onset of decomposition, in both $\mathbf{8 a}$ and $\mathbf{8} \mathbf{b}$ suggests that $\mathbf{8} \mathbf{b}$ can be transformed into $8 \mathrm{a}$ by removing the solvate acetonitrile.

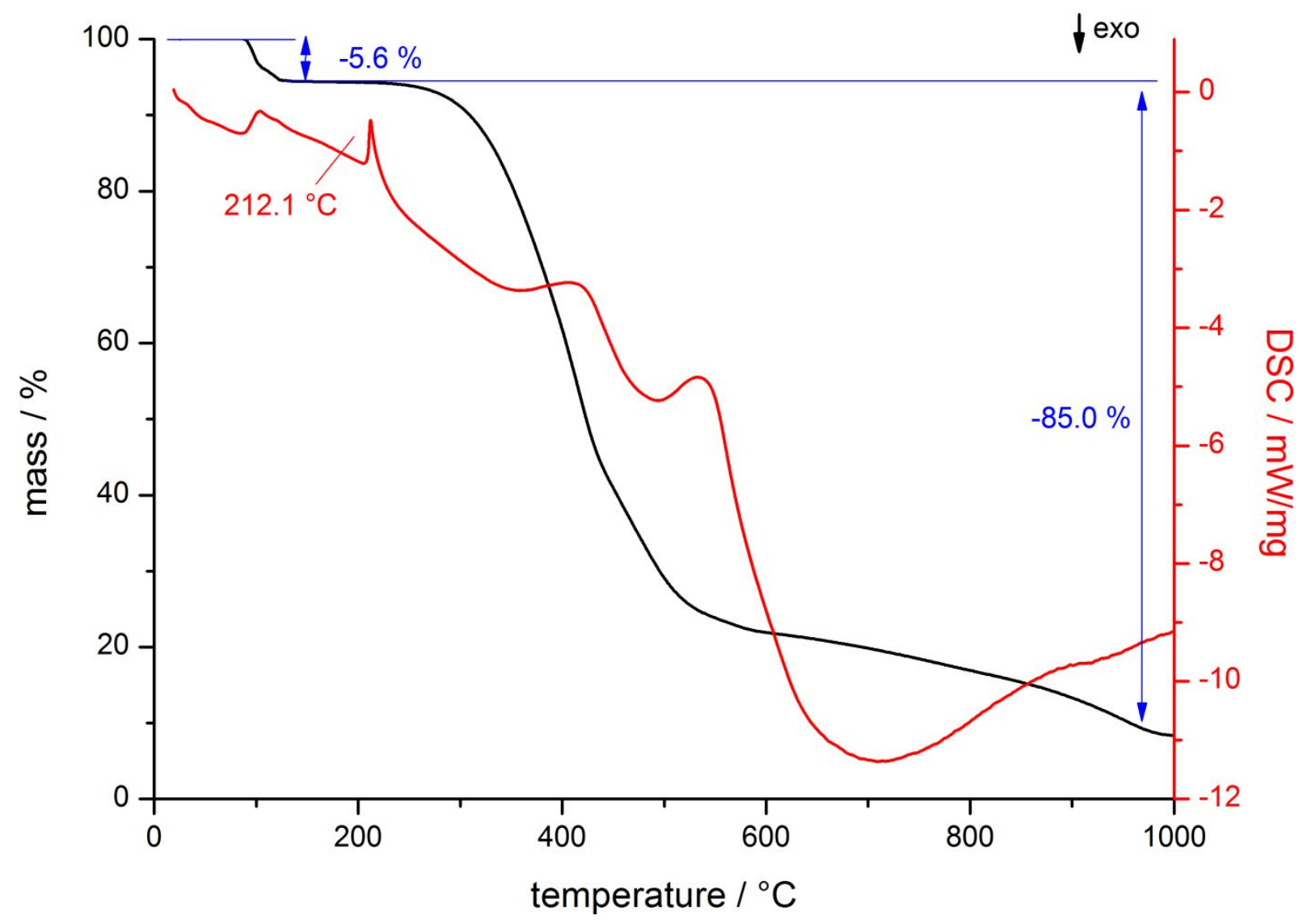

Figure S19. TGA/DSC of $\mathbf{8 b .}$ 
The thermal behavior of $\mathbf{9 b}(17.6 \mathrm{mg})$, was studied by TGA/DSC on a NETSCH STA $409 \mathrm{C} / \mathrm{CD}$ from $25^{\circ} \mathrm{C}$ to $1000^{\circ} \mathrm{C}$ with a heating rate of $10^{\circ} \mathrm{C} \mathrm{min}^{-1}$ in a constant flow of $80 \mathrm{ml} \mathrm{min}-1 \mathrm{~N}_{2}$.

A first mass loss of $5.3 \%$ is observed starting at $100{ }^{\circ} \mathrm{C}$, corresponding to the loss of 4 molecules of acetonitrile per formula unit. A second mass loss starts at $200{ }^{\circ} \mathrm{C}$ and corresponds to the decomposition of the compound, possibly involving the loss of $\mathrm{PPh}_{3}$ and $\mathrm{Bil}_{3}$. These results suggest that $\mathbf{9 b}$ can be desolvated to form $9 a$, yet as our experiments show, it is difficult to do this without partial decomposition. It may be possible to desolvate $\mathbf{9 b}$ by keeping the sample at temperatures well below $100^{\circ} \mathrm{C}$ in vacuum for extended periods of time, possibly with repeated grindings, but these experiments are beyond the scope of the work presented here. The endothermic peak at $209.6^{\circ} \mathrm{C}$ likely corresponds to a melting point concomitant with the onset of decomposition.

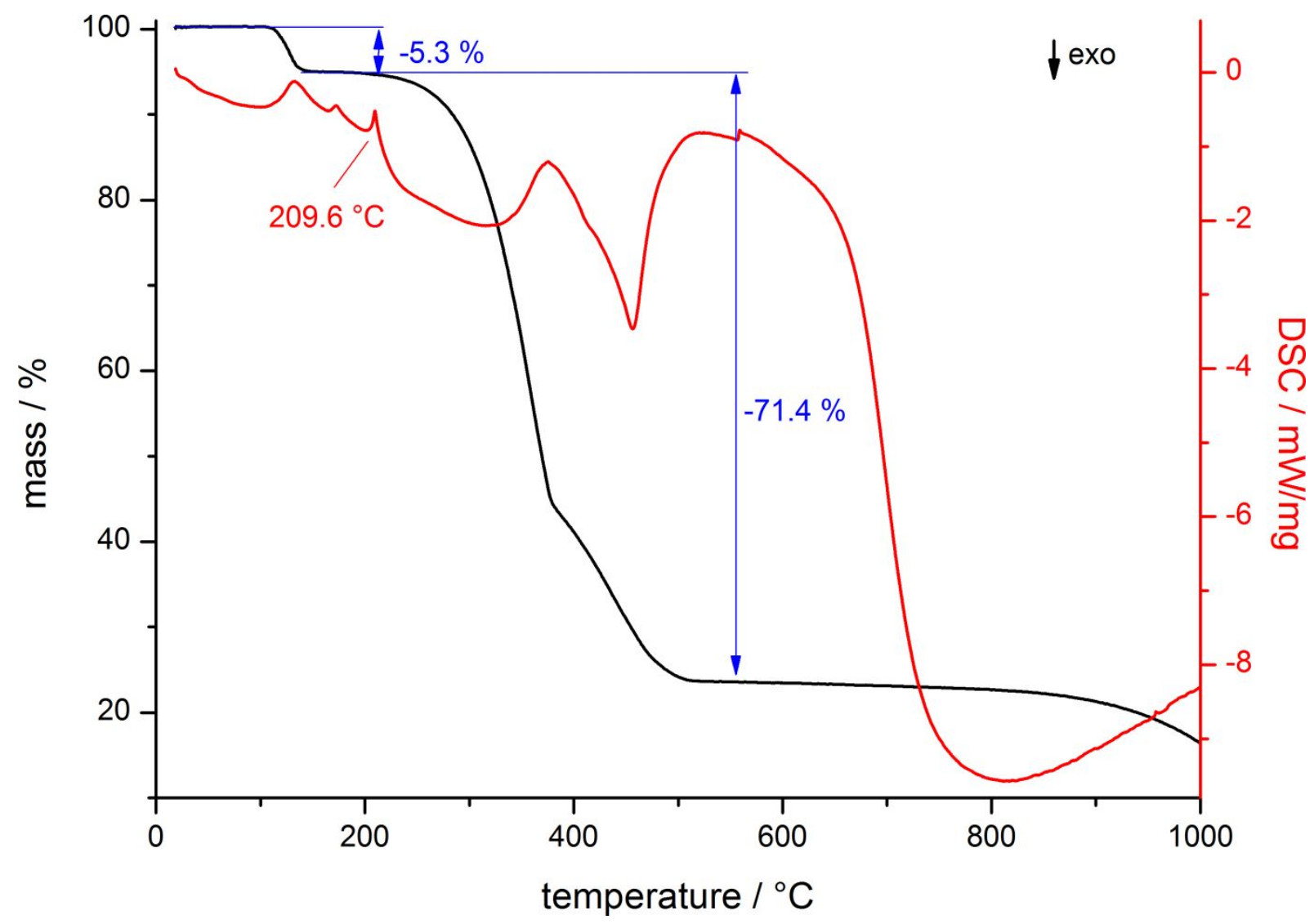

Figure S20. TGA/DSC of $\mathbf{9 b}$. 


\section{Powder diffraction}

Powder patterns were recorded on a STADI MP (STOE Darmstadt) powder diffractometer, with $\mathrm{CuK}_{\alpha 1}$ radiation with $\lambda=1.54056 \AA$ at room temperature in transmission mode from 3 to $80^{\circ}$ in $2 \theta$, although in the patterns shown below no major reflections are found beyond $50^{\circ}$ in $2 \theta$. The patterns confirm the presence of the phase determined by SCXRD measurement and the absence of any major crystalline by-products except in case of compound 1 (see details below). For compounds $\mathbf{4}$ and $\mathbf{5 a}$, the thermal decomposition products obtained after the TGA measurement were also investigated (see Figure S33 and S34).

Investigation of the desolvation product of $\left[\mathrm{Bi}_{2} \mathrm{Cu}_{2} \mathrm{Cl}_{8}\left(\mathrm{PPh}_{3}\right)_{4}\left(\mathrm{Me}_{2} \mathrm{CO}\right)_{2}\right] \cdot 2 \mathrm{Me}_{2} \mathrm{CO}(1)$

$\mathrm{CHN}$ after washing and drying (expected for $\left.\left[\mathrm{Bi}_{2} \mathrm{Cu}_{2} \mathrm{Cl}_{8}\left(\mathrm{PPh}_{3}\right)_{4}\left(\mathrm{Me}_{2} \mathrm{CO}\right)_{2}\right] \cdot 0.5 \mathrm{Me}_{2} \mathrm{CO}\right)$ : $\mathrm{C} 47.23$ (47.200), $\mathrm{H} 3.699$ (3.737) indicates a partial loss of solvate acetone (1.5 molecules per formula unit). The powder pattern indicates a complete transformation from 1 to the desolvation product. The lack of a significant amorphous background and the relatively simple pattern suggests that a single product is obtained in the process. A triclinic unit cell of dimensions $a=13.04103 \AA ; b=15.71783 \AA$; $c=10.21261 \AA$; $\alpha=$ $93.9508^{\circ} ; b=105.4106^{\circ} ; \nu=81.0936^{\circ}$ can be determined and refined from the diffraction pattern using WINXPOW $3.07^{6}$ and JANA20067.

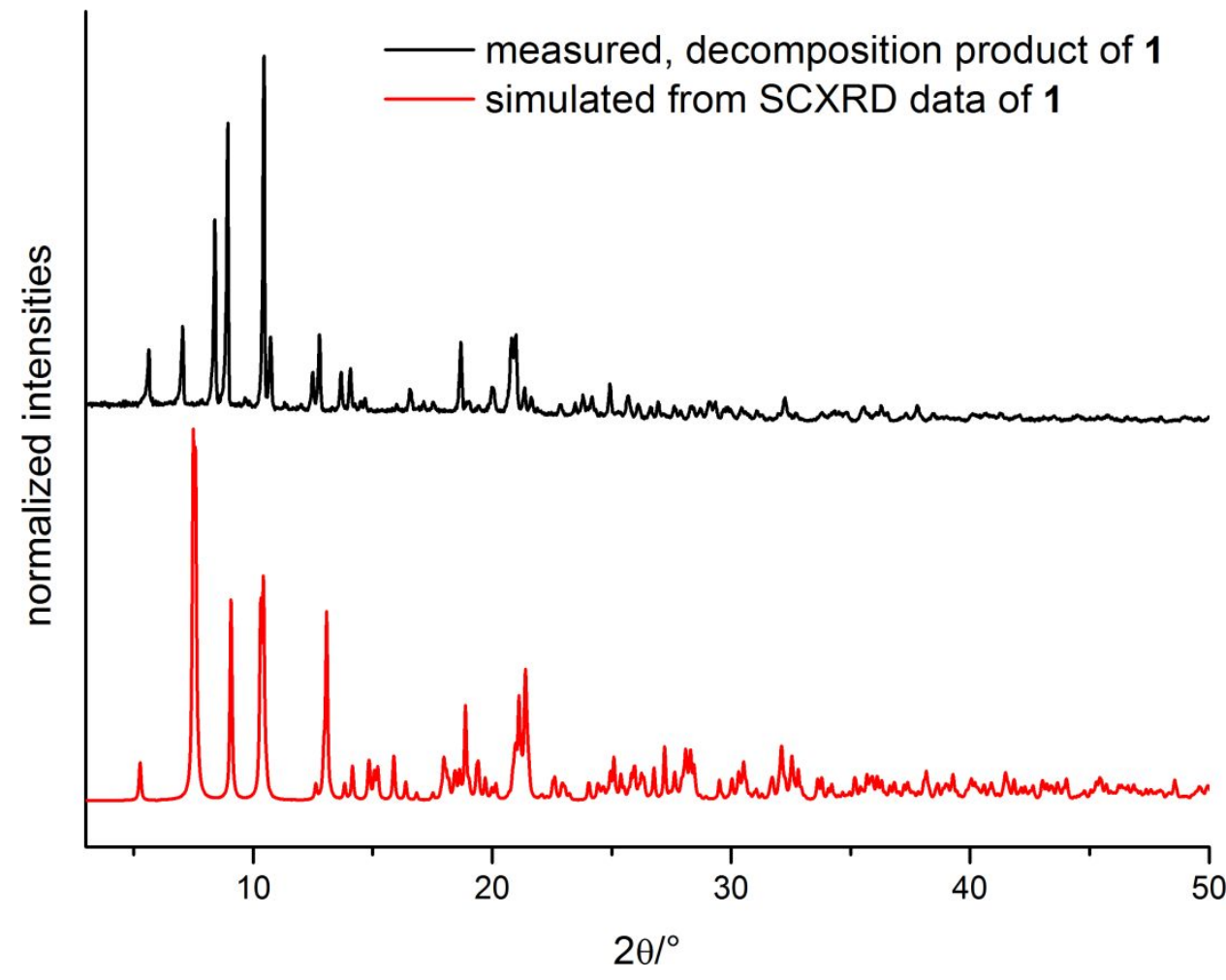

Figure S21: Powder diffraction pattern of the decomposition product of 1. 


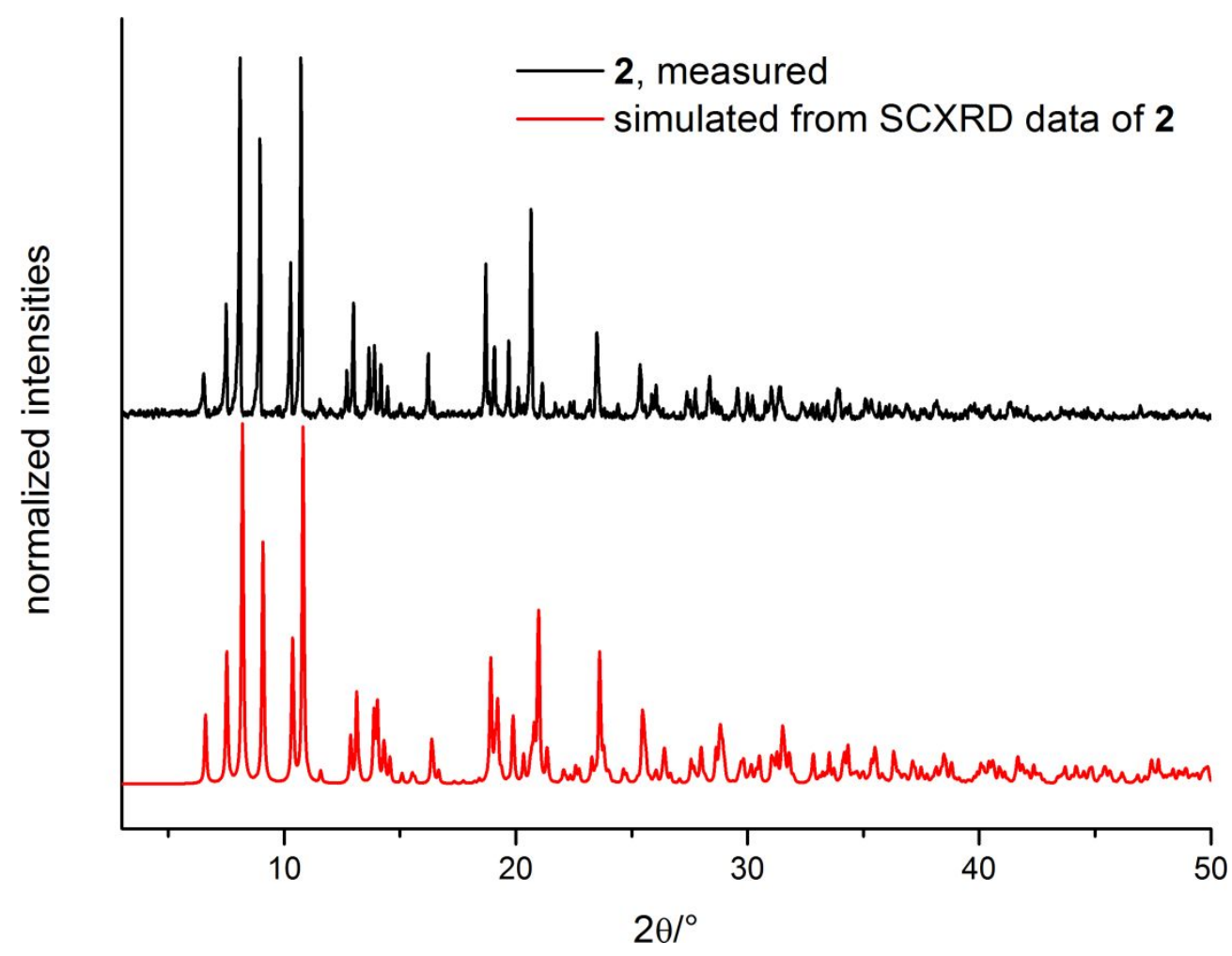

Figure S22. Powder diffraction pattern of compound 2.

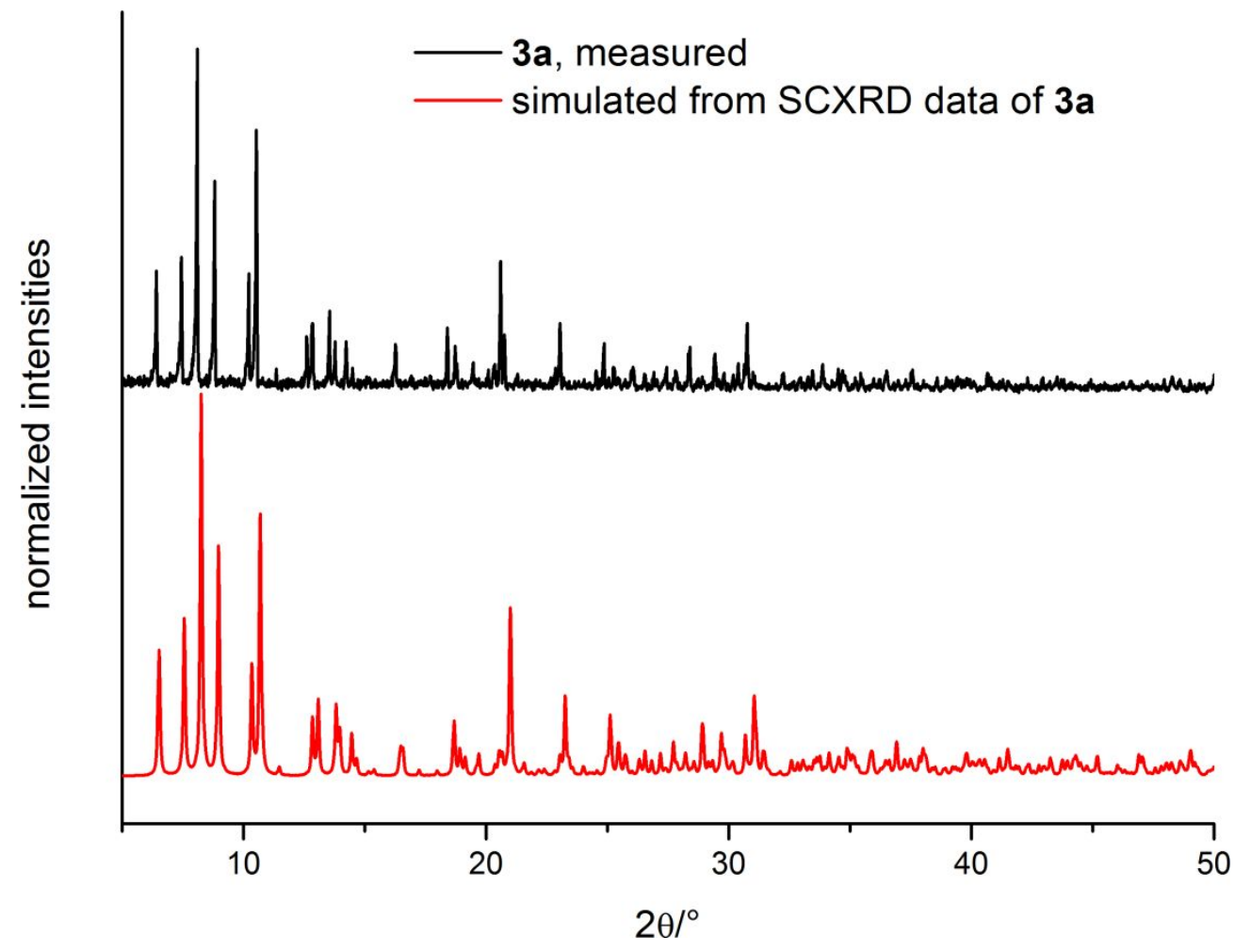

Figure S23. Powder diffraction pattern of compound 3a. 


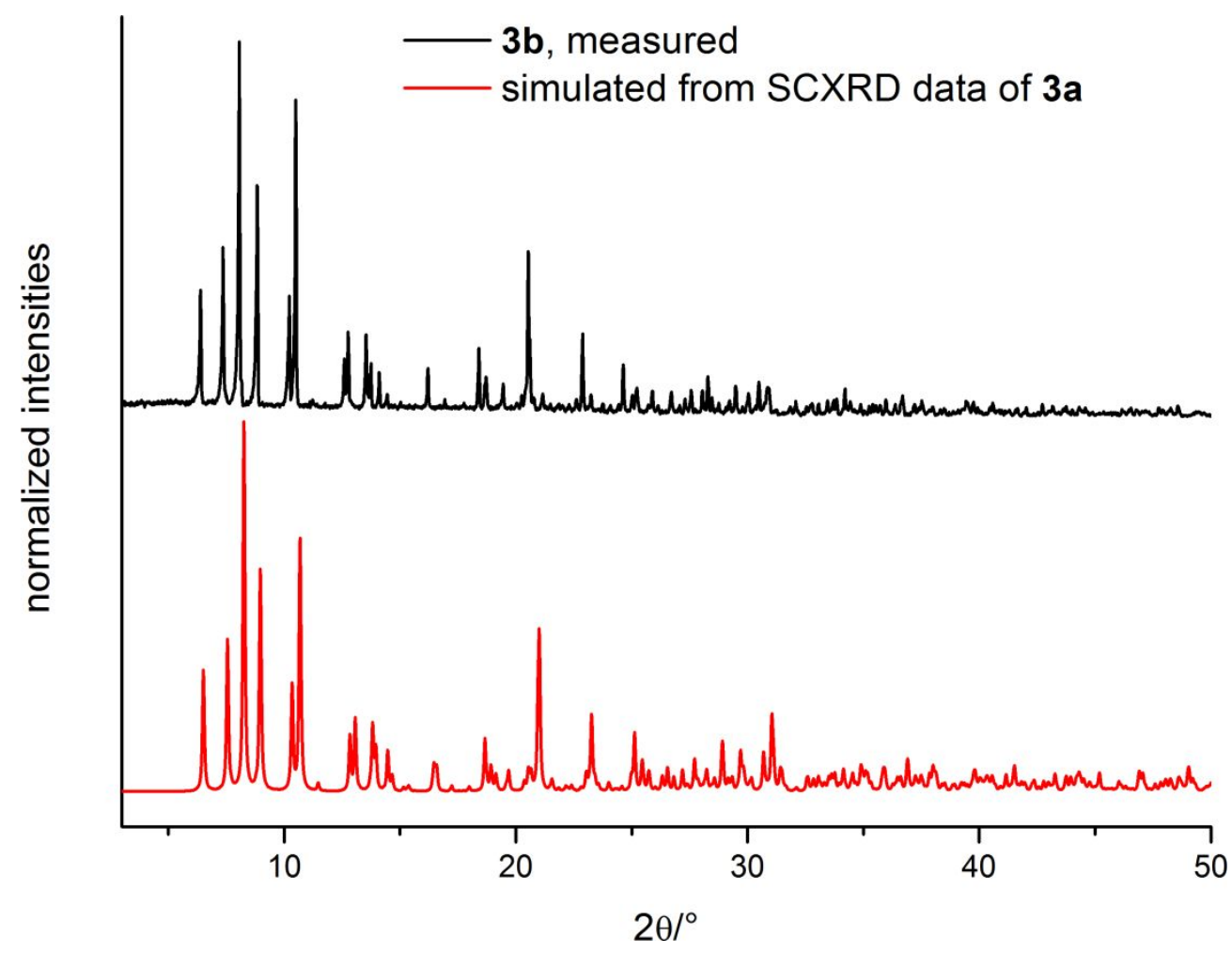

Figure S24. Powder diffraction pattern of compound 3b.

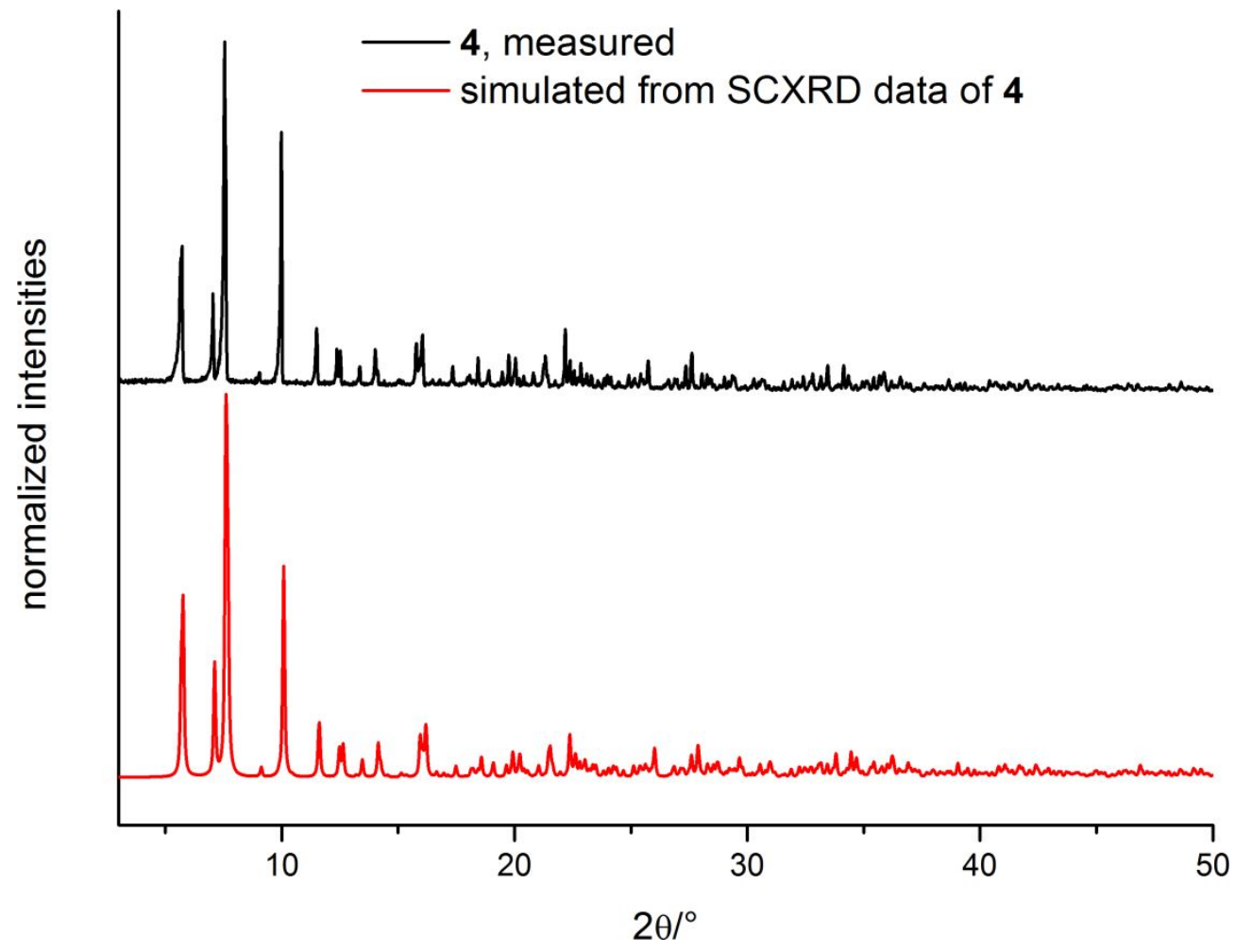

Figure S25. Powder diffraction pattern of compound 4. 


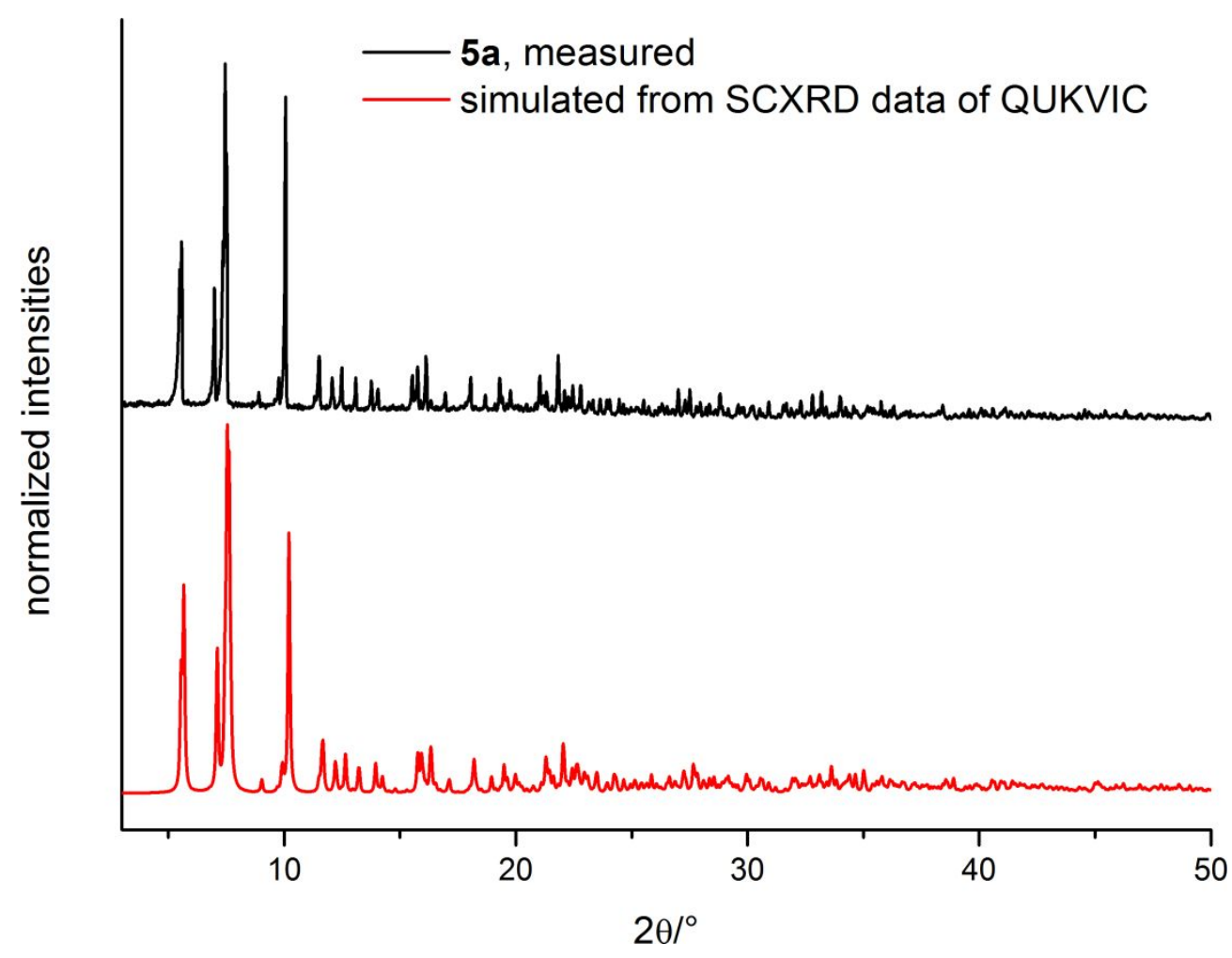

Figure S26. Powder diffraction pattern of compound 5a, SCXRD data taken from Fenske's work ${ }^{3}$ CCDC data entry QUKVIC.

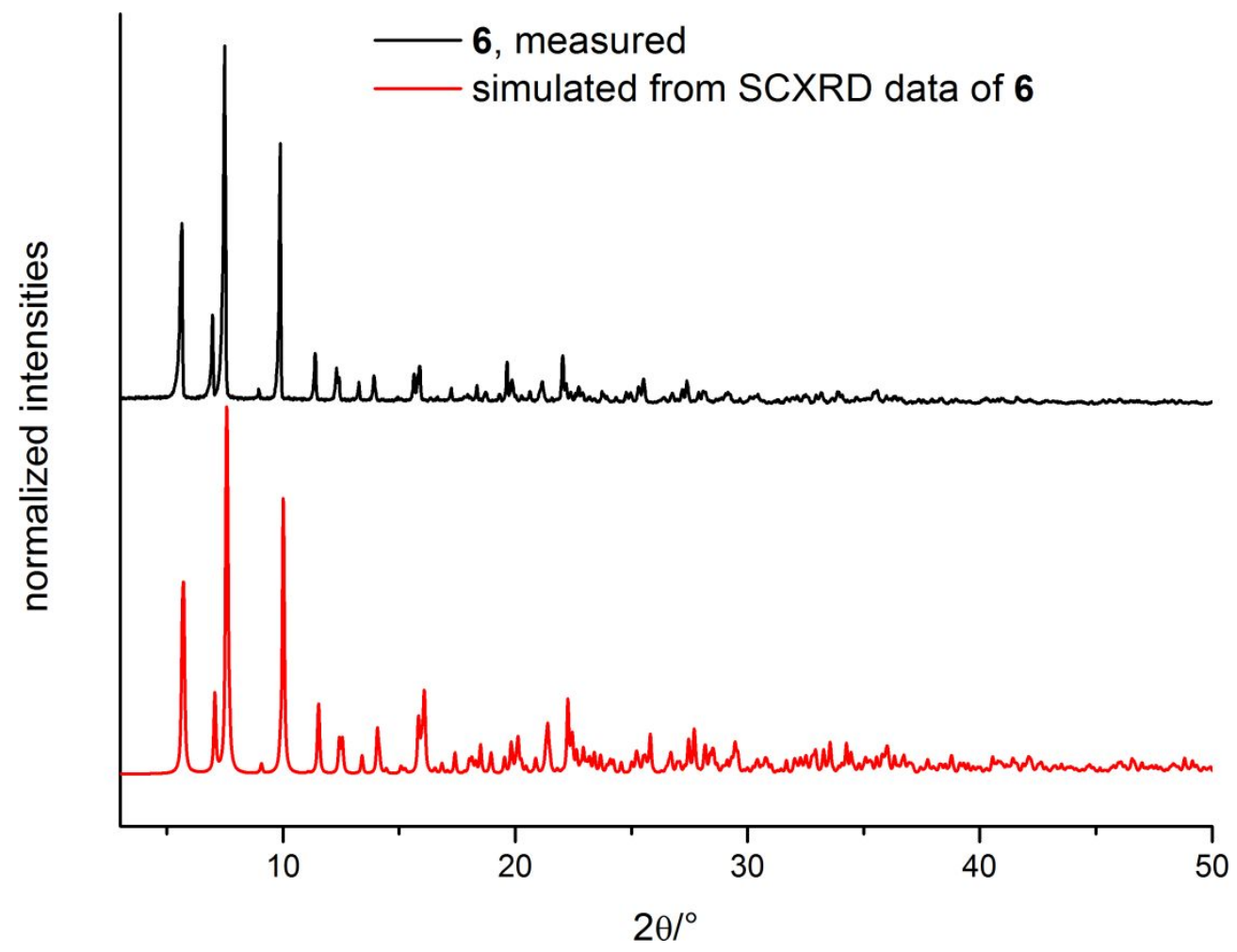

Figure S27. Powder diffraction pattern of compound 6. 


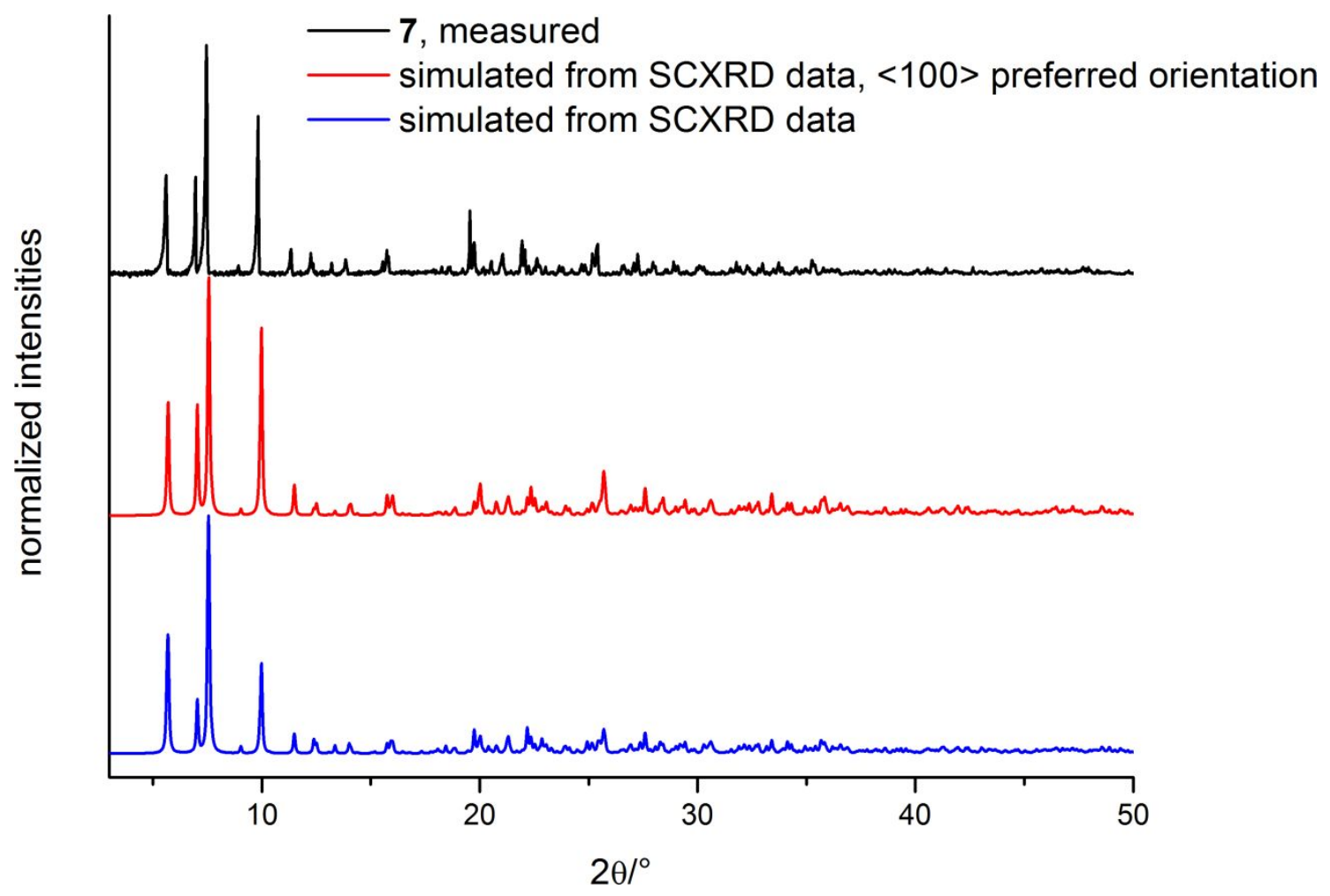

Figure S28. Powder diffraction pattern of compound 7. A texture effect with a $<100>$ preferred orientation is observed here.

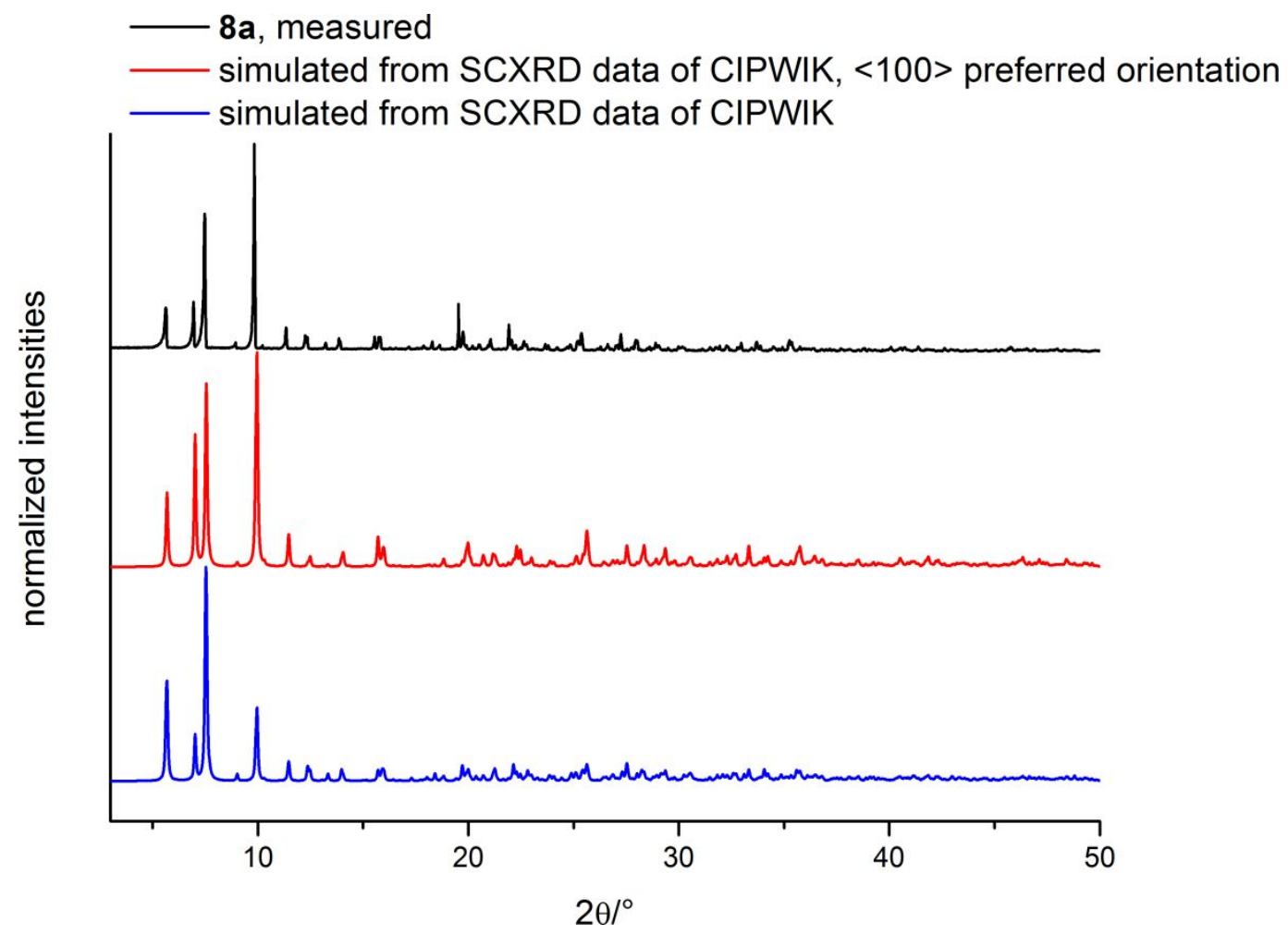

Figure S29. Powder diffraction pattern of compound 8a. The rod-like habit induces a texture effect with a $<100>$ preferred orientation. SCXRD data taken from Pike's work ${ }^{6} /$ CCDC data entry CIPWIK. 


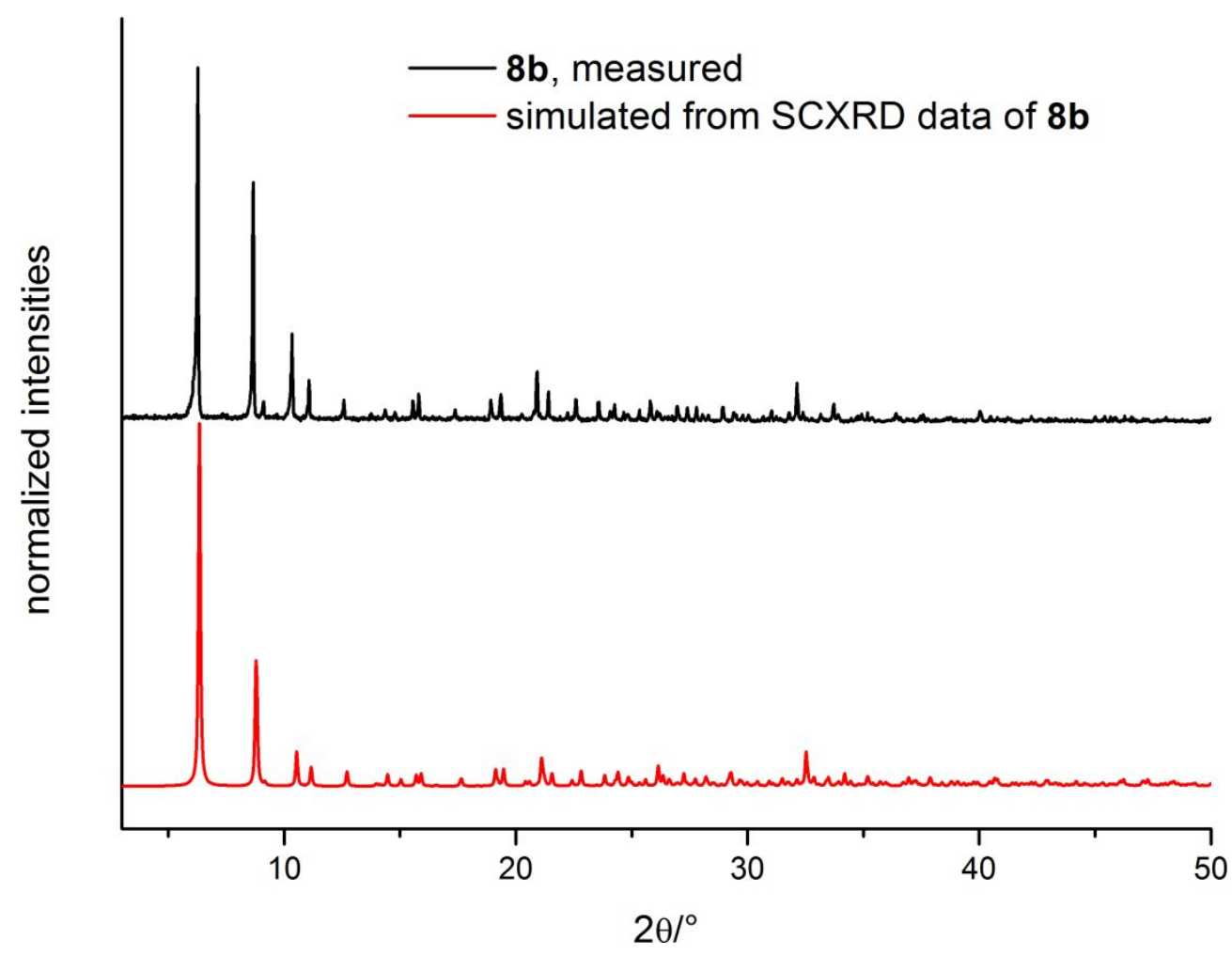

Figure S30. Powder diffraction pattern of compound 8a.

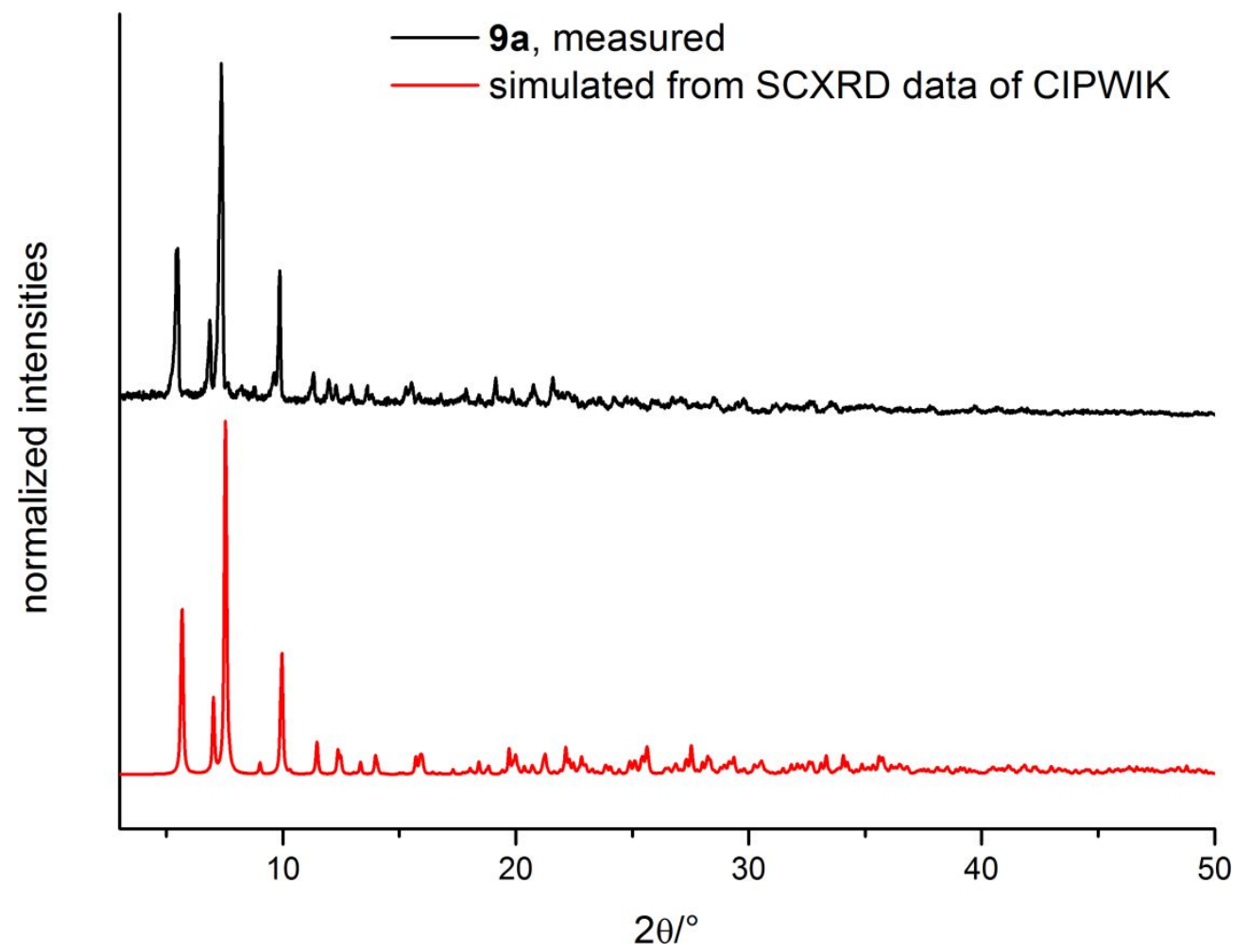

Figure S31. Powder diffraction pattern of compound 9a. SCXRD data of the isostructural copper compound taken from Pike's work ${ }^{6}$ / CCDC data entry CIPWIK. 


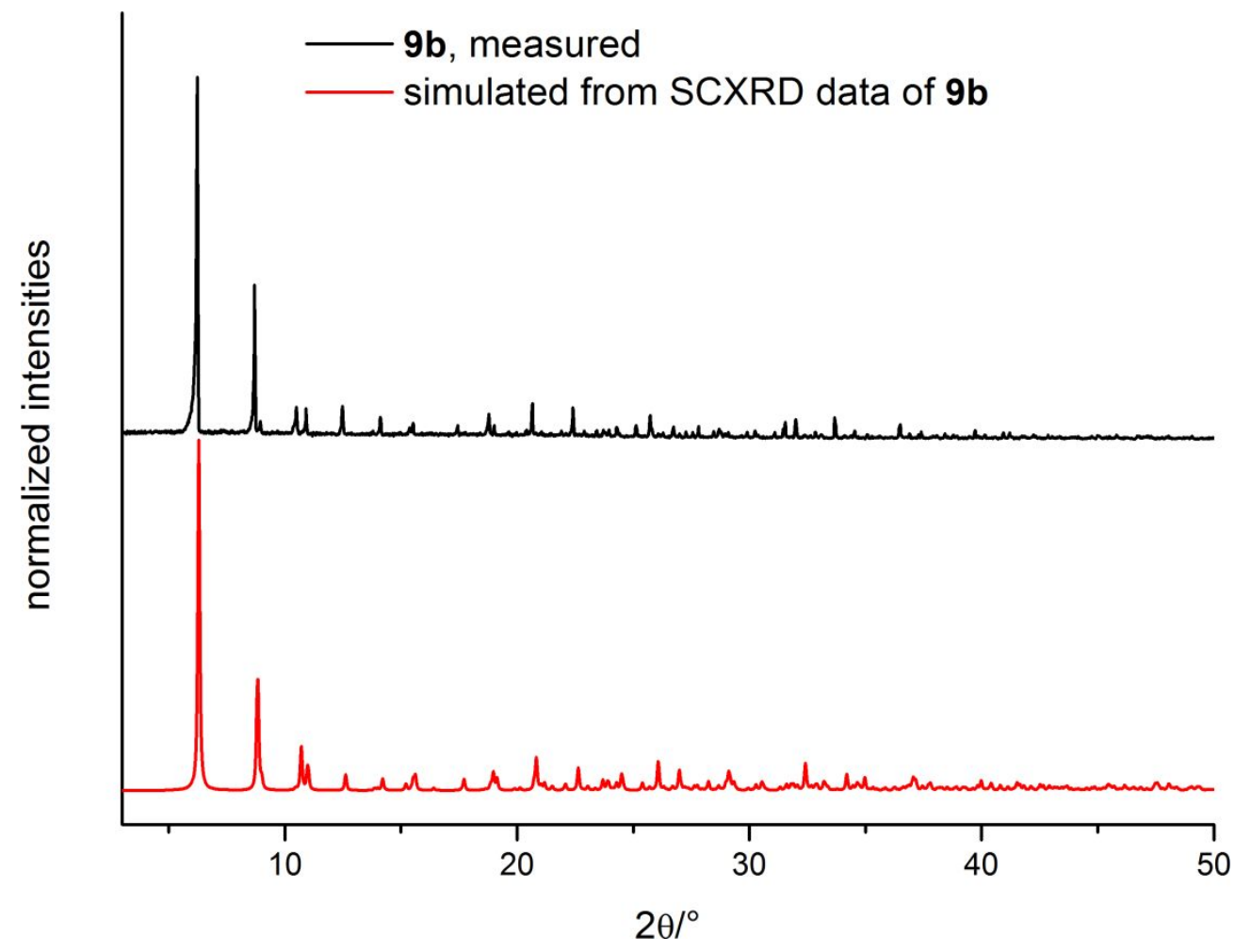

Figure S32. Powder diffraction pattern of compound $\mathbf{9 b}$.

Investigation of the thermal decomposition product of $\left[\mathrm{BiCu}_{3} \mathrm{Br}_{6}\left(\mathrm{PPh}_{3}\right)_{6}\right](4)$

The powder pattern of the TGA residue of $\mathbf{4}$ shows that $\mathrm{Cu}_{3} \mathrm{P}$ is obtained as a crystalline product and indicates the presence of additional amorphous phases. 


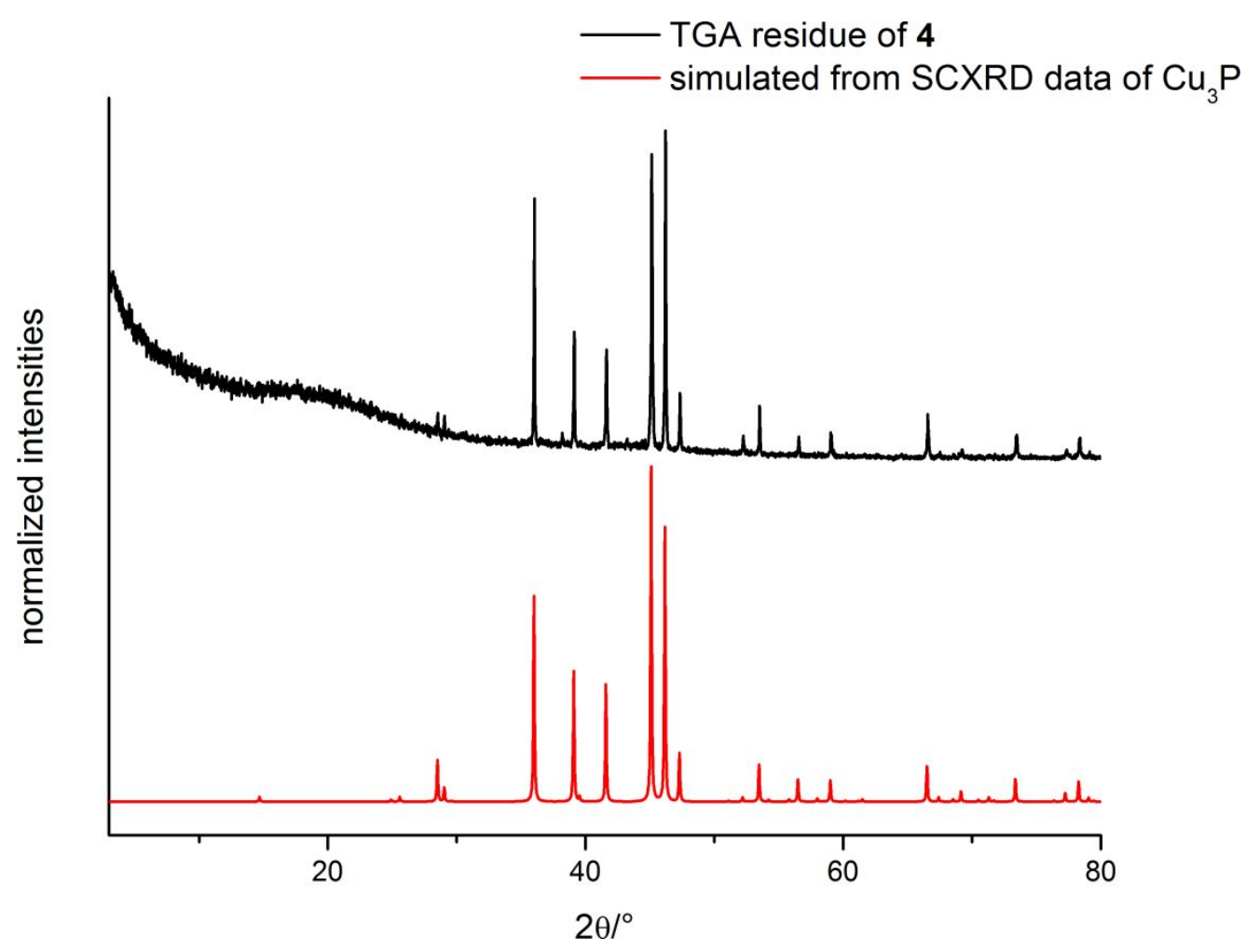

Figure S33: Powder diffraction pattern of the thermal decomposition product of 4, data for $\mathrm{Cu}_{3} \mathrm{P}$ taken from reference 9.

Investigation of the thermal decomposition product of $\left[\mathrm{BiAg}_{3} \mathrm{Br}_{6}\left(\mathrm{PPh}_{3}\right)_{6}\right](5 \mathrm{a})$

The powder pattern of the TGA residue of $\mathbf{5}$ shows that $\mathrm{Ag}$ and $\mathrm{AgBr}$ are obtained as the crystalline products, although additional amorphous phases are also present. 


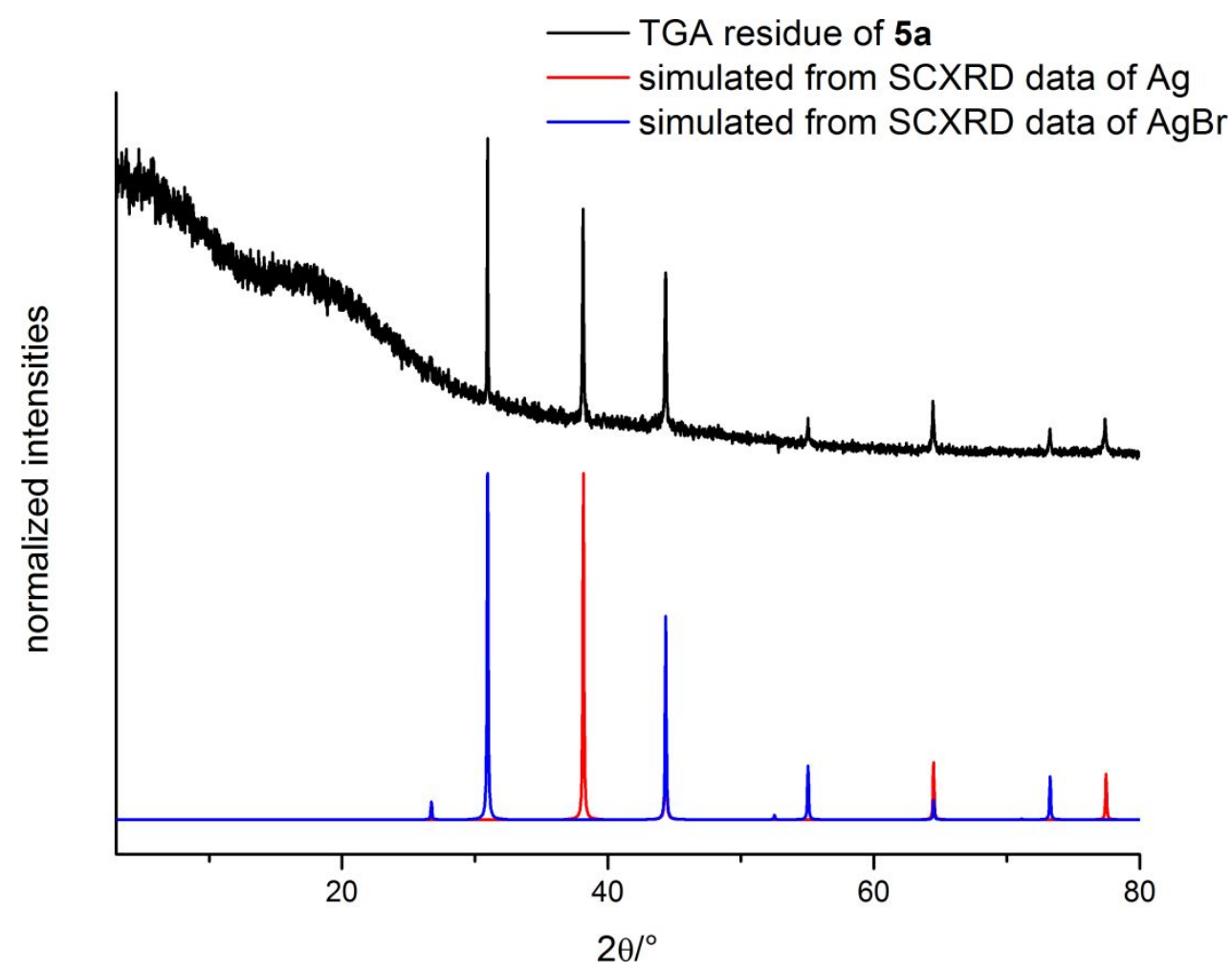

Figure S34: Powder diffraction pattern of the thermal decomposition product of 5a, data for $\mathrm{Ag}$ and $\mathrm{AgBr}$ taken from reference 10 and 11. 


\section{IR spectroscopy}

IR spectra were recorded on a Bruker Tensor 37 FT-IR spectrometer equipped with an ATRPlatinum measuring unit.

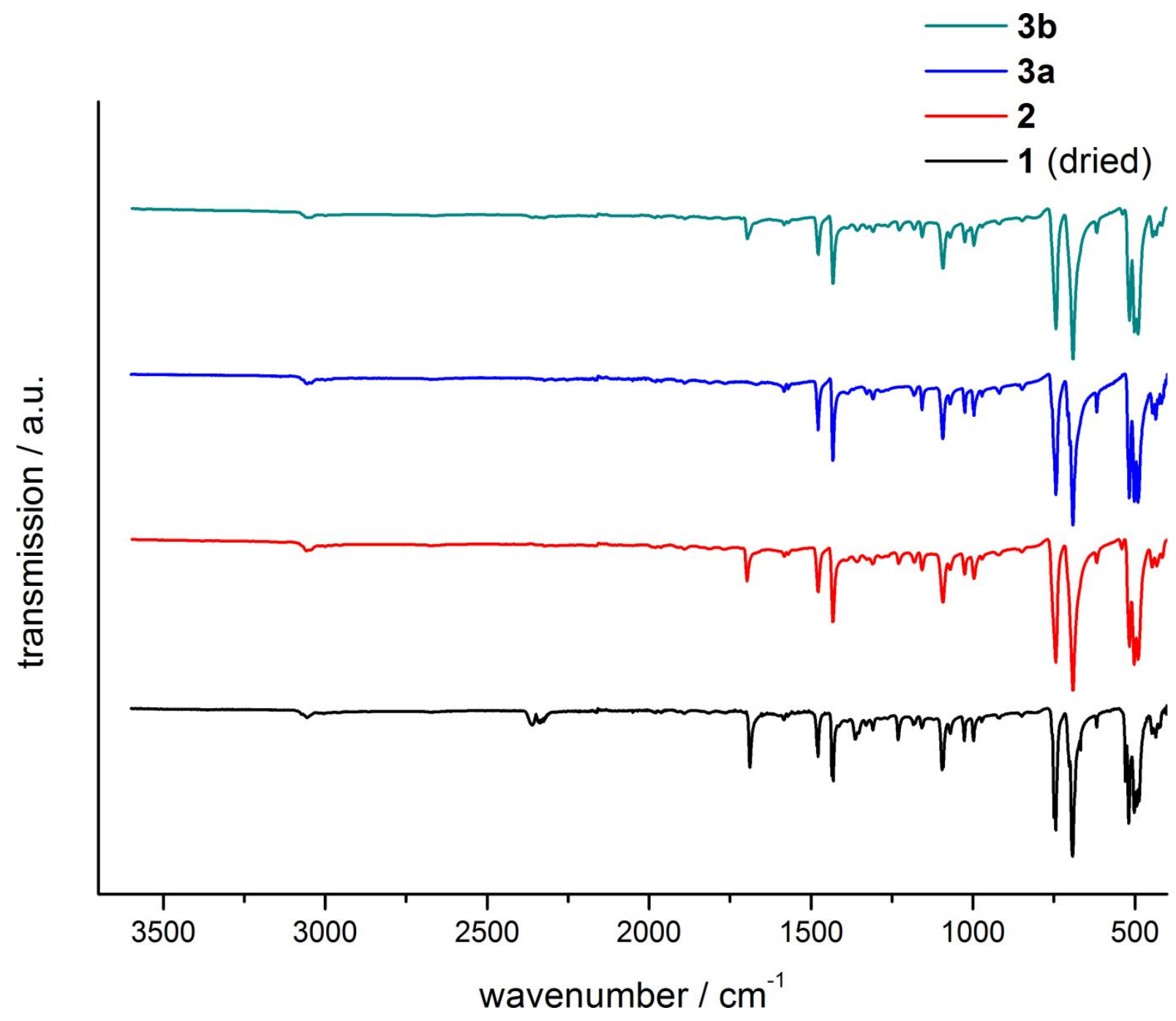

Figure S35. IR spectra of compound $\mathbf{1}$ (after drying), 2, 3a and $\mathbf{3 b}$. Spectra are dominated by bands of the $\mathrm{PPh}_{3}$ ligands ${ }^{12}$ and the $\mathrm{C}=\mathrm{O}$ band of acetone at ca. $1700 \mathrm{~cm}^{-1}$ for $\mathbf{1}, \mathbf{2}$ and $\mathbf{3 b}$, while the $\mathrm{C} \equiv \mathrm{N}$ band expected at ca. $2200 \mathrm{~cm}^{-1}$ in $\mathbf{3 a}$ is not clearly observed. 


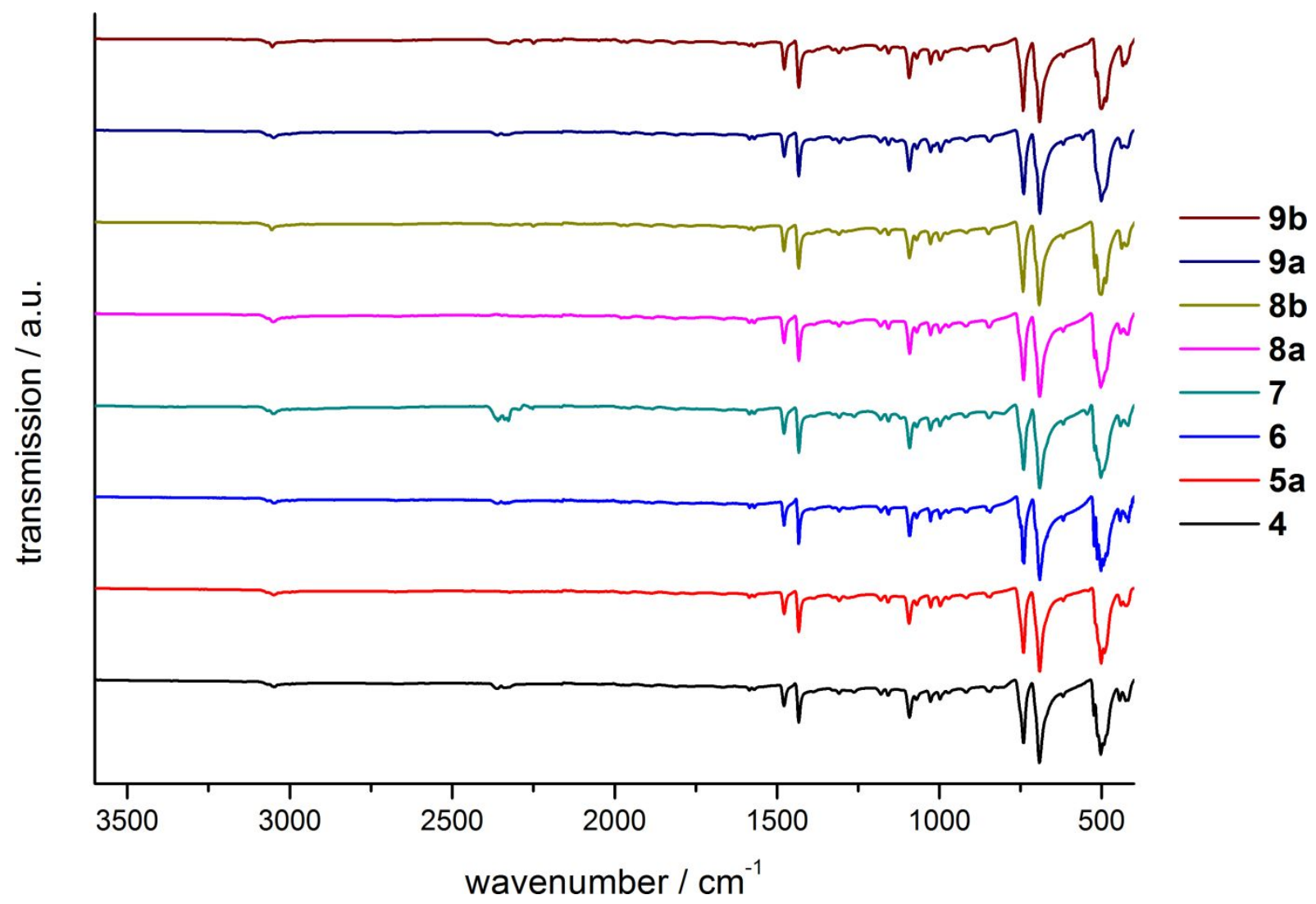

Figure S36. IR spectra of compound 4-9b. Spectra are dominated by bands of the $\mathrm{PPh}_{3}$ ligands, while the $\mathrm{C} \equiv \mathrm{N}$ band expected at ca. $2200 \mathrm{~cm}^{-1}$ in $\mathbf{8 b}$ and $\mathbf{9 b}$ is not clearly observed.

\section{Optical properties}

Optical absorption spectra were recorded on a Varian Cary 5000 UV/Vis/NIR spectrometer in the range of 300-800 $\mathrm{nm}$ in diffuse reflectance mode employing a Praying Mantis accessory (Harrick). For ease of viewing, raw data was transformed from \%Reflectance $R$ to Absorbance $A$ according to $A=\log (1 / R) \cdot{ }^{13}$ 


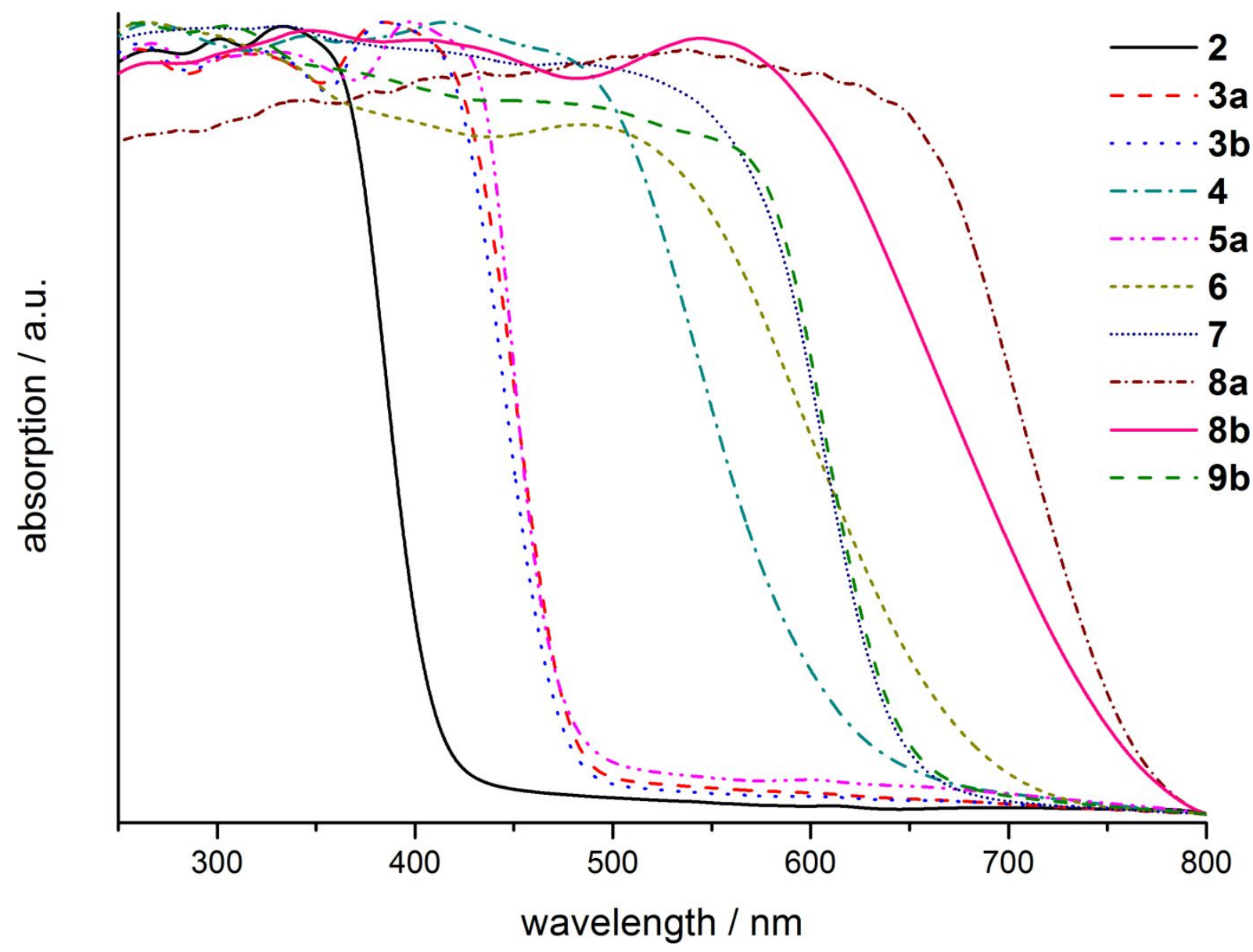

Figure S37. Optical absorption spectra of all compounds prepared as pure phases, measured in diffuse reflectance.

\section{Crystal Pictures}

Pictures were taken using a Zeiss SteREO Discovery.V8 optical microscope. 


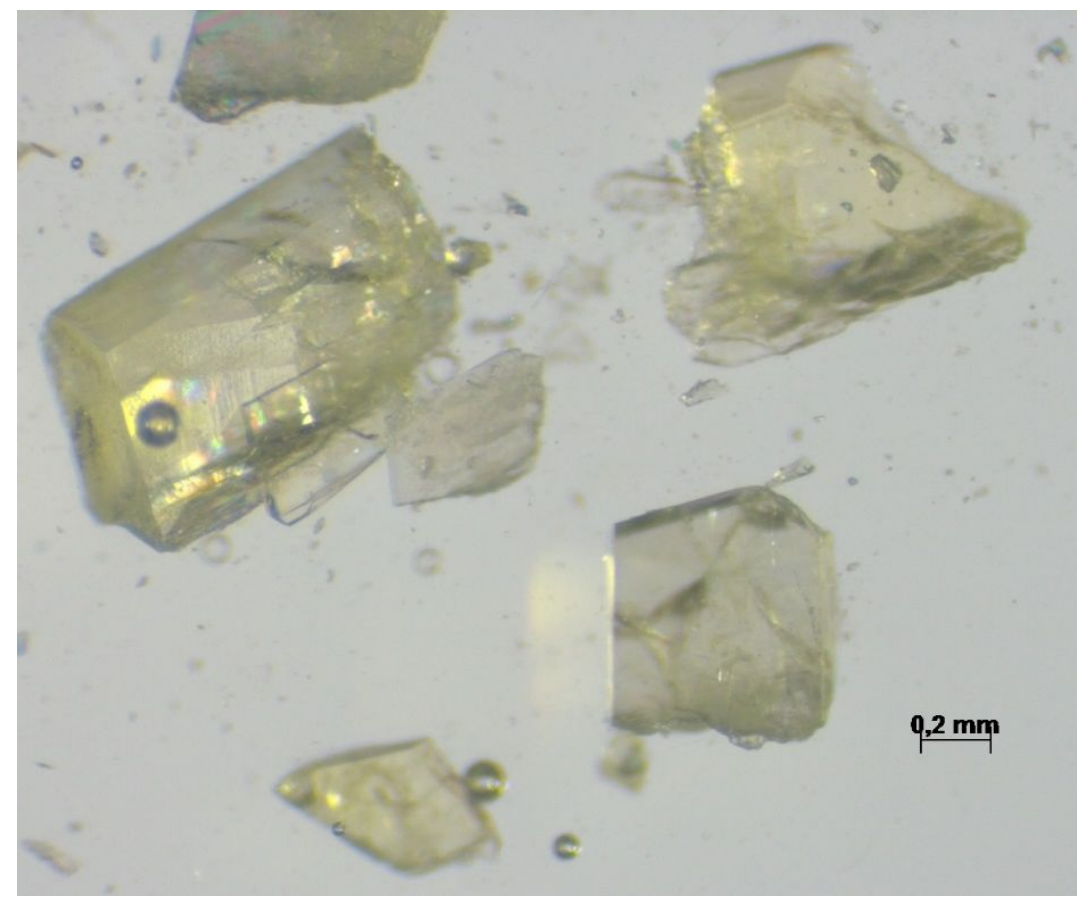

Figure S38. Picture of crystals of $\left[\mathrm{Bi}_{2} \mathrm{Cu}_{2} \mathrm{Cl}_{5}\left(\mathrm{PPh}_{3}\right)_{4}\left(\mathrm{Me}_{2} \mathrm{CO}\right)\right] \cdot 2 \mathrm{Me}_{2} \mathrm{CO}(\mathbf{1})$.

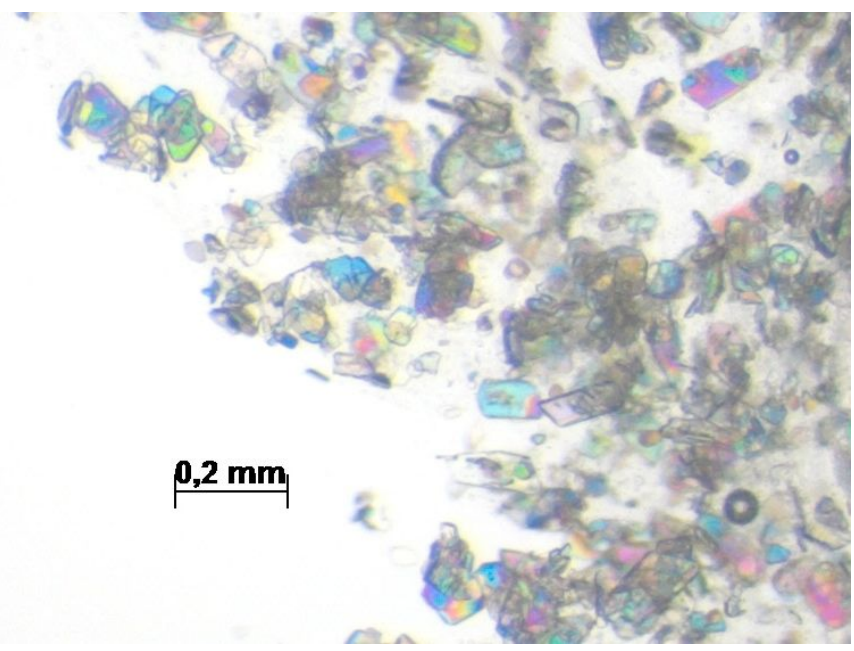

Figure S39. Picture of crystals of $\left[\mathrm{BiAg}_{2} \mathrm{Cl}_{5}\left(\mathrm{PPh}_{3}\right)_{4}\left(\mathrm{Me}_{2} \mathrm{CO}\right)\right]$ (2). 


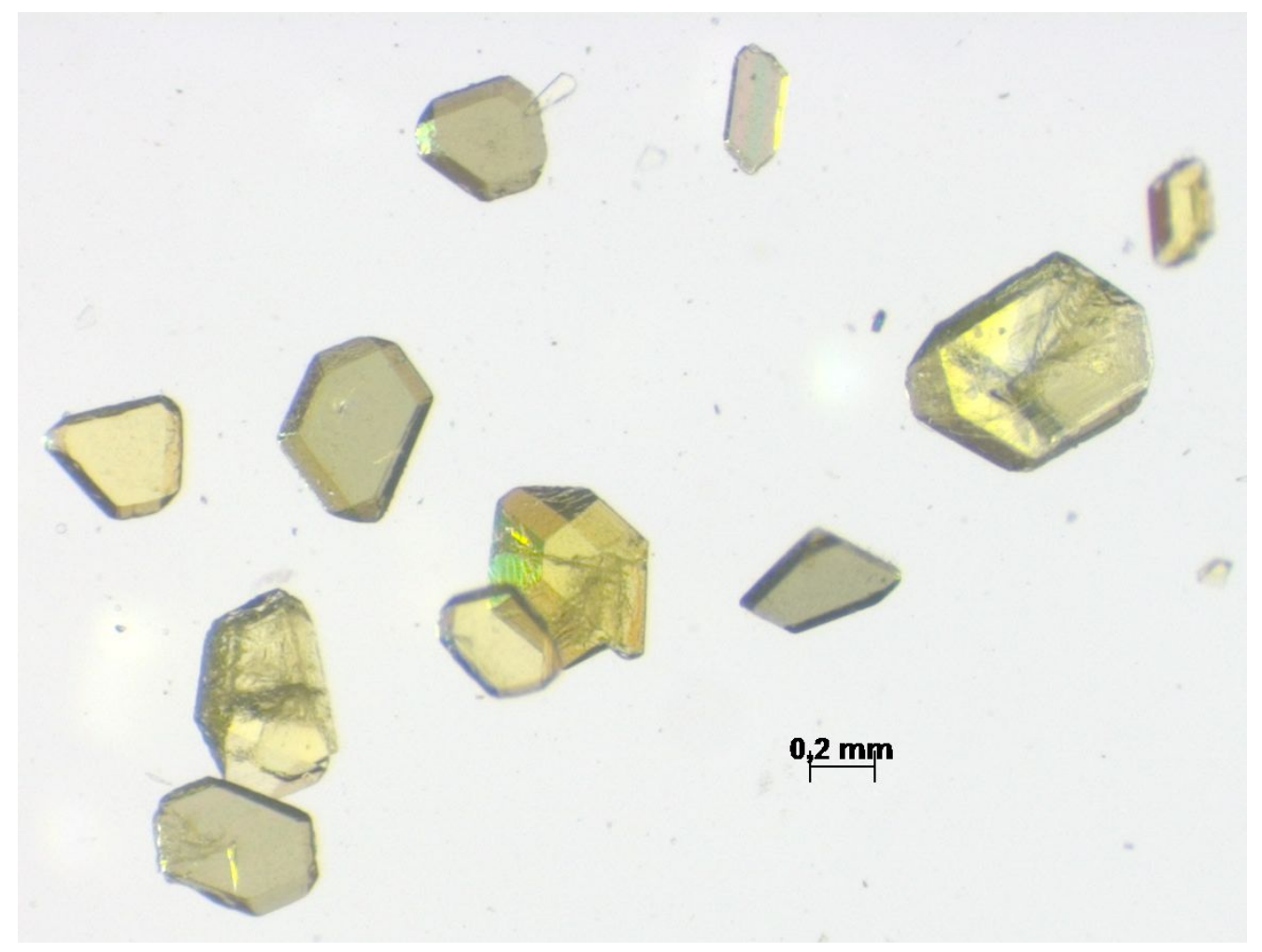

Figure S40. Picture of crystals of $\left[\mathrm{BiAg}_{2} \mathrm{Br}_{5}\left(\mathrm{PPh}_{3}\right)_{4}(\mathrm{MeCN})\right](3 \mathrm{a})$.

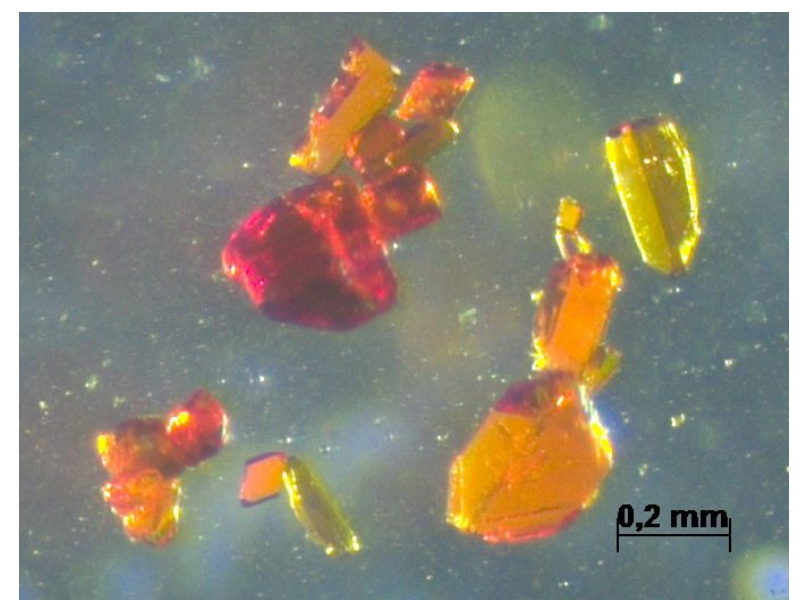

Figure S41. Picture of crystals of $\left[\mathrm{BiCu}_{3} \mathrm{Br}_{6}\left(\mathrm{PPh}_{3}\right)_{6}\right](4)$. 


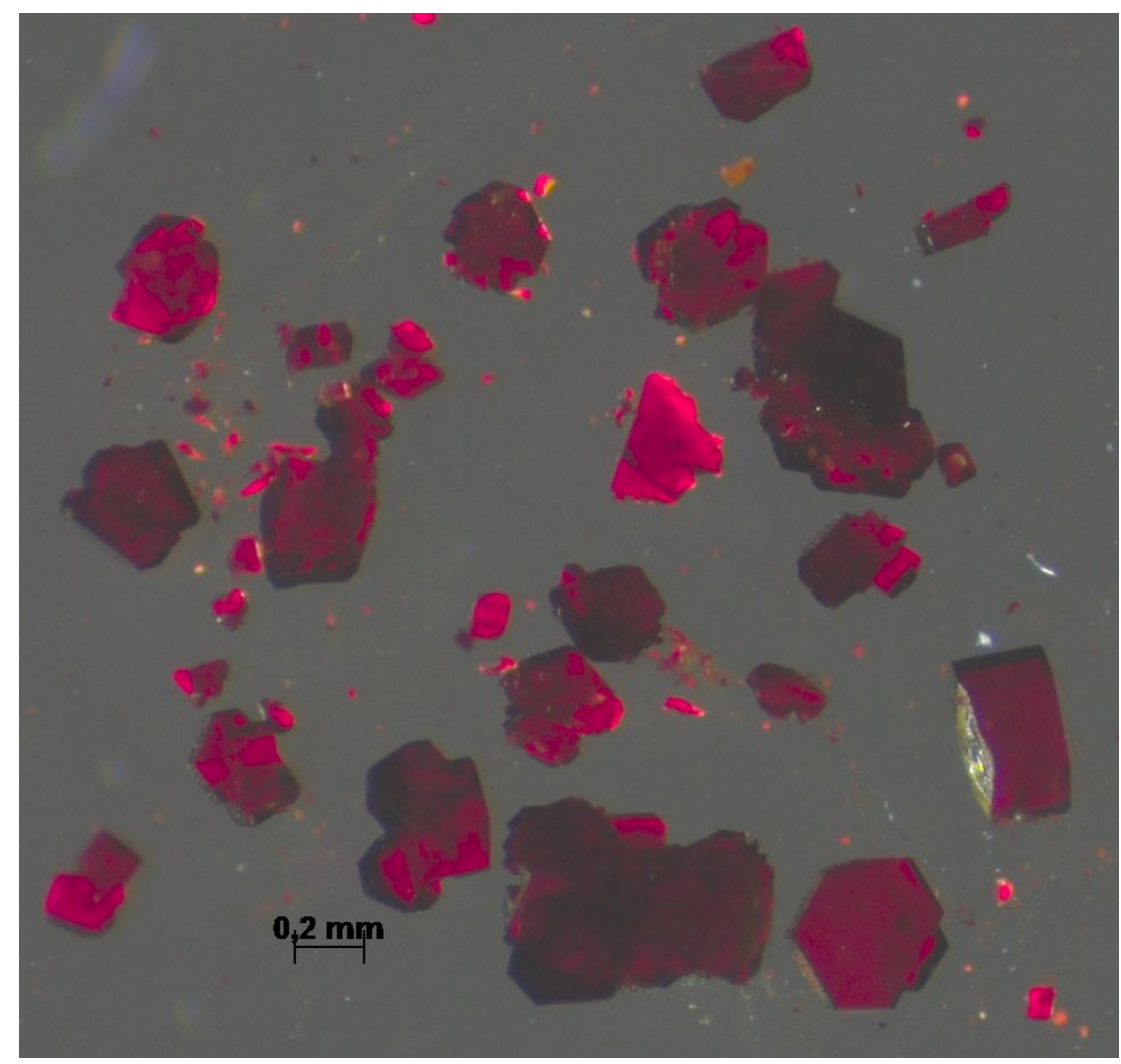

Figure S42. Picture of crystals of $\left[\mathrm{BiCu}_{3} \mathrm{I}_{3} \mathrm{Br}_{3}\left(\mathrm{PPh}_{3}\right)_{6}\right](6)$.

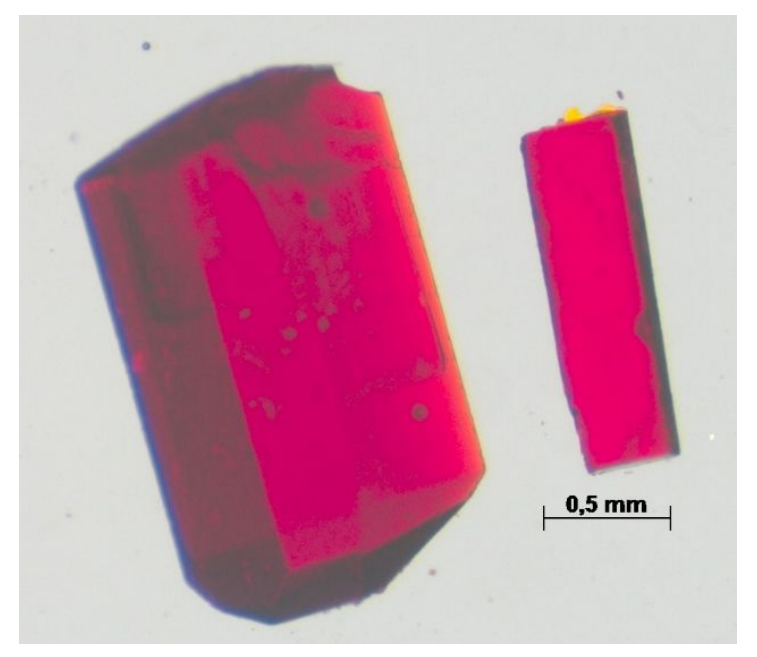

Figure S43. Picture of crystals of $\left[\mathrm{SbCu}_{3} \mathrm{I}_{6}\left(\mathrm{PPh}_{3}\right)_{6}\right](7)$. 


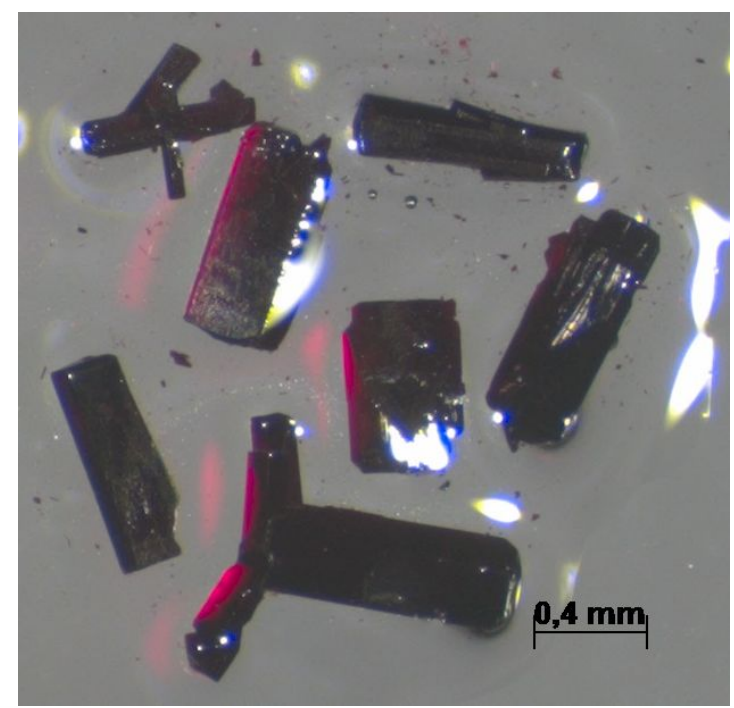

Figure S44. Picture of crystals of $\left[\mathrm{BiCu}_{3} \mathrm{I}_{6}\left(\mathrm{PPh}_{3}\right)_{6}\right](8 \mathrm{a})$.

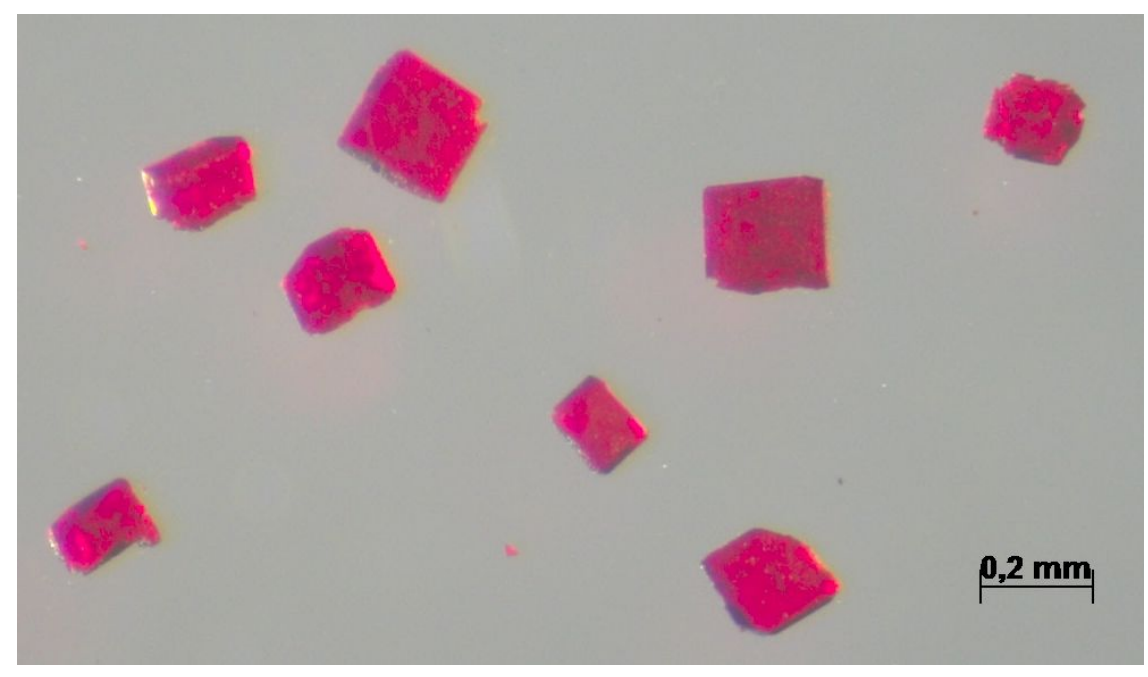

Figure S45. Picture of crystals of $\left[\mathrm{BiAg}_{3} \mathrm{I}_{6}\left(\mathrm{PPh}_{3}\right)_{6}\right] \cdot 4 \mathrm{MeCN}(\mathbf{9 b})$.

\section{References}

1 Brauer, G., Ed. Handbuch der Präparativen Anorganischen Chemie, 3rd ed., Vol. 1; Ferdinand Enke Verlag: Stuttgart, Germany, 1975, 591-592.

2 Brauer, G., Ed. Handbuch der Präparativen Anorganischen Chemie, 3rd ed., Vol. 1; Ferdinand Enke Verlag: Stuttgart, Germany, 1975, 600.

3 Sommer, H.; Eichhöfer, A.; Fenske, D. Synthesis, Crystal Structure, and Thermal

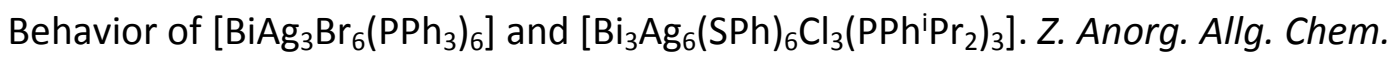
2009, 635, 1997-2001. 
4 Bowmaker, G. A.; Effendy; Hart, R. D.; Kildea, J. D.; White, A. H. Lewis-Base Adducts of Group 11 Metal(I) Compounds. LXXIII Synthesis, Spectroscopy and Structural Systematics of New 1: 1 'Cubane' Tetramers of Copper(I) and Silver(I) Halides with Triphenylarsine. Aust. J. Chem. 1997, 50, 653-670.

5 Stiefel, E. I.; Brown, G. F. Detailed nature of the six-coordinate polyhedra in tris (bidentate ligand) complexes. Inorg. Chem. 1972, 11, 434-436.

6 Kelly, A. W.; Wheaton, A. M.; Nicholas, A. D.; Barnes, F. H.; Patterson, H. H.; Pike, R. D. lodobismuthate(III) and lodobismuthate(III)/lodocuprate(I) Complexes with Organic Ligands. Eur. J. Inorg. Chem. 2017, 4990-5000.

7 WINXPOW 3.3.0.1, Stoe \& Cie GmbH, Darmstadt, Germany, 2015.

8 Petricek, V.; Dusek, M.; Palatinus, L. Crystallographic Computing System JANA2006: General features. Z. Kristallogr. 2014, 229, 345-352.

9 Demchyna, R. O.; Chykhrij, S. I.; Kuz'ma, Yu. B. Y-Cu-P system. J. Alloys Compd. 2002, $345,170-174$.

10 Zeiringer, I.; Grytsiv, A.; Bauer, E.; Giester, G.; Rogl, P. Phase Relations and Crystal Structures in the Ternary Systems Sr-\{Ag, Au\}-\{Si, Ge\}. Z. Anorg. Allg. Chem. 2015, $641,1404-1421$.

11 A. G. Mikolaichuk, N. V. Moroz, P. Yu. Demchenko, L. G. Akselrud, R. E. Gladyshevskii, Phase relations in the $\mathrm{Ag}_{8} \mathrm{SnS}_{6}-\mathrm{Ag}_{2} \mathrm{SnS}_{3}-\mathrm{AgBr}$ system and crystal structure of $\mathrm{Ag}_{6} \mathrm{SnS}_{4} \mathrm{Br}_{2}$. Inorg. Mater. 2010, 46, 590-597.

12 Kuchen, W.; Buchwald, H. Zur Kenntnis der Organophosphorverbindungen, II. Das Tetraphenyldiphosphin. Chem. Ber. 1958, 91, 2871-2877.

13 Boldish, S. I.; White, W. B. Optical band gaps of selected ternary sulfide minerals. Am. Mineral. 1998, 83, 865-871. 